\title{
System Size and Energy Dependence of Dilepton Production in Heavy-Ion Collisions at 1-2 $A \mathrm{GeV}$ Energies
}

\author{
E.L. Bratkovskaya, ${ }^{1,2}$ J. Aichelin, ${ }^{3}$ M. Thomere ${ }^{3}$ S. Vogel,${ }^{1}$ and M. Bleicher ${ }^{1,2}$ \\ ${ }^{1}$ Institute for Theoretical Physics, Frankfurt University, 60438 Frankfurt-am-Main, Germany \\ ${ }^{2}$ Frankfurt Institut for Advanced Studies, Frankfurt University, 60438 Frankfurt-am-Main, Germany \\ ${ }^{3}$ SUBATECH, Laboratoire de Physique Subatomique et des Technologies Associées, \\ Université de Nantes - IN2P3/CNRS - Ecole des Mines de Nantes \\ 4 rue Alfred Kastler, F-44072 Nantes, Cedex 03, France
}

(Dated: August 6, 2018)

\begin{abstract}
We study the dilepton production in heavy-ion collisions at energies of $1-2 A \mathrm{GeV}$ as well as in proton induced $p p, p n, p d$ and $p+A$ reactions from $1 \mathrm{GeV}$ up to $3.5 \mathrm{GeV}$ where data have been taken by the HADES collaboration. For the analysis we employ three different transport models the microscopic off-shell Hadron-String-Dynamics (HSD) transport approach, the Isospin Quantum Molecular Dynamics (IQMD) approach as well as the Ultra-relativistic Quantum Molecular Dynamics (UrQMD) approach. We find that the HSD and IQMD models describe very reasonably the elementary $p p, p n$ and $\pi N$ reactions despite of different assumptions on quantities like the excitation function of the $\Delta$ multiplicity, where solid experimental constraints are not available. Taking these data on elementary collisions as input, the three models provide a good description of the presently available heavy ion data. In particular, we confirm the experimentally observed enhancement of the dilepton yield (normalized to the multiplicity of neutral pions $N_{\pi^{0}}$ ) in heavy-ion collisions with respect to that measured in $N N=(p p+p n) / 2$ collisions. We identify two contributions to this enhancement: a) the $p N$ bremsstrahlung which scales with the number of collisions and not with the number of participants, i.e. pions; b) the dilepton emission from intermediate $\Delta$ 's which are part of the reaction cycles $\Delta \rightarrow \pi N ; \pi N \rightarrow \Delta$ and $N N \rightarrow N \Delta ; N \Delta \rightarrow N N$. With increasing system size more generations of intermediate $\Delta$ 's are created. If such $\Delta$ decays into a pion, the pion can be reabsorbed, however, if it decays into a dilepton, the dilepton escapes from the system. Thus, experimentally one observes only one pion (from the last produced $\Delta$ ) whereas the dilepton yield accumulates the contributions from all $\Delta$ 's of the cycle. We show as well that the Fermi motion enhances the production of pions and dileptons in the same way. Furthermore, employing the off-shell HSD approach, we explore the influence of in-medium effects like the modification of self-energies and spectral functions of the vector mesons due to their interactions with the hadronic environment. We find only a modest influence of the in-medium effects on the dilepton spectra in the invariant mass range where data with small error bars exist.
\end{abstract}

PACS numbers: 25.75.-q, 25.40.-h

\section{INTRODUCTION}

According to the theory of strong interactions, the Quantum Chromo Dynamics (QCD), hadrons are bound objects of quarks and gluons. The properties of hadrons in vacuum are well known and confirmed by lattice QCD calculations [1] while the properties of hadrons in a strongly interacting environment are subject of intensive research. QCD inspired approaches as well as phenomenological models based on phase shifts and SU(3) symmetry [1 14] predict significant changes of hadron properties in a strongly interacting medium. The results of the different models vary substantially. It is therefore one of the challenges of novel experimental heavy-ion physics to study these in-medium modifications of hadrons. Besides the in-medium properties of the antikaon, interesting also for astrophysical reasons, the vector mesons and especially the $\rho$ meson have been in the focus of the theoretical interest because the $\rho$ has the quantum numbers of a photon and can therefore disintegrate into an electron-positron pair. Having only electromagnetic interactions this pair may easily leave the reaction region without further collisions. This allows to reconstruct the invariant mass of the decaying $\rho^{0}$. Thus there is a hope that by measuring the dilepton invariant mass spectra the in-medium mass and width of the $\rho$ meson become experimentally accessible. For this it is necessary to separate the background from known dilepton sources - Dalitz decays of baryonic and mesonic resonances as well as $p N$ and $\pi N$ bremsstrahlung. At SPS energies of 40 and $158 \mathrm{AGeV}$ such an enhancement above the known background has been measured by the CERES [15] and the NA60 [16] collaborations. The experimental results are compatible with the assumption that in the medium the peak position of the $\rho$ meson mass distribution remains rather unchanged while the width increases considerably (cf. [17 22]).

At much lower energies, at energies around $1 \mathrm{AGeV}$ dileptons have been measured in heavy-ion collisions at the BEVALAC in Berkeley by the DLS Collaboration [23 26]. These data led to the so called 'DLS puzzle' because the DLS dilepton yield in $\mathrm{C}+\mathrm{C}$ and $\mathrm{Ca}+\mathrm{Ca}$ collisions in the invariant mass range from 0.2 to $0.5 \mathrm{GeV}$ [26] was about five 
times higher than the results from different transport models at that time using the 'conventional' dilepton sources such as bremsstrahlung, $\pi^{0}, \eta, \omega$ and $\Delta$ Dalitz decays and direct decay of vector mesons $(\rho, \omega, \phi)$ [27 29]. This discrepancy remained even after including in the transport calculations the different scenarios for the in-medium modifications of vector meson properties, as dropping mass or collisional broadening of the $\rho$ and $\omega$ spectral functions 30 33. To solve this puzzle was one of the main motivations to build the HADES (High Acceptance Dilepton Spectrometer) detector at GSI in Germany [34, 34 39]. In 2008 the HADES collaboration confirmed the DLS data [34, 35] for C+C at 1.0 $A \mathrm{GeV}$. In the mean time also the theoretical transport approaches as well as effective models for the elementary $N N$ reactions have been further developed. As it has been suggested in Ref. [40], the DLS puzzle can be solved when incorporating stronger $p n$ and $p p$ bremsstrahlung contributions in line with the updated One-Boson-Exchange (OBE) model calculations from [41]. The previous OBE approaches [42] used in the old transport calculations for the analysis of the DLS data, gave results close to the soft photon approximation. As shown in Ref. [40] the results of the HSD model (off-shell microscopic Hadron-String-Dynamics (HSD) transport approach) with 'enhanced' bremsstrahlung cross sections agree very well with the HADES experimental data for $\mathrm{C}+\mathrm{C}$ at 1 and $2 A \mathrm{GeV}$ as well as with the DLS data for $\mathrm{C}+\mathrm{C}$ and $\mathrm{Ca}+\mathrm{Ca}$ at $1 \mathrm{AGeV}$, especially when one includes a collisional broadening in the vector-meson spectral functions. Similar results have been obtained by other, independent transport approaches - IQMD [45] and the Rossendorf BUU [46].

Despite of the fact that theory predicts that the vector meson properties are modified substantially already at energies as low as 1-2 AGeV, it is quite difficult to observe these changes experimentally. The production yield of $\rho^{0}$ and $\omega$ mesons is small at these energies and the background from other dilepton sources like $\Delta$-Dalitz decay and $p N$ bremsstrahlung is large in the mass range of interest, $M>0.4 \mathrm{GeV}$. Therefore the presently available data do not allow for a detailed investigation of the in-medium properties of vector mesons.

This focuses the interest of the present studies to the question whether the invariant mass spectrum below the $\rho / \omega$ peak depends on the system size and on the beam energy in a non-trivial way, i.e. whether the dilepton invariant mass spectra can be understood as a superposition of individual $p p$ and $p n$ interactions. In a first publication [37] the HADES collaboration found that the invariant mass spectra of dileptons, observed in 1 and $2 A \mathrm{GeV} \mathrm{C}+\mathrm{C}$ collisions, are practically coincident below $M=0.4 \mathrm{GeV}$ if divided by the total number of observed $\pi^{0}$ and after subtracting the $\eta$ Dalitz decay contribution. It is strongly suppressed at $1 A \mathrm{GeV}$ but becomes essential at $2 A \mathrm{GeV}$ due to the kinematical threshold for the $\eta$ production in $N N$ collisions. This scaling with the $\pi^{0}$ number can be interpreted as a scaling with the number of participants $N_{\text {part }}$. The HADES collaboration, comparing the dilepton yield from the light $\mathrm{C}+\mathrm{C}$ systems with the elementary $p p$ and $p d$ interactions, albeit taken at different energies, has concluded that the dilepton invariant mass spectra in these light systems can be considered as a mere superposition of $p n$ and $p p$ collisions without any 'in-medium' enhancement.

In a more recent publication [39] a heavier system, $\mathrm{Ar}+\mathrm{KCl}$ at $1.75 \mathrm{AGeV}$, was investigated and the collaboration came to the conclusion that in this reaction the dilepton invariant mass yield between 0.2 and $0.6 \mathrm{GeV}$ is about $2-3$ times larger than expected from a mere superposition of $p p$ and $p n$ collisions [39]. From the analysis of the excess in the transverse-mass slope and from the angular anisotropies the HADES collaboration concluded that the excess of dileptons in the low invariant mass region scales with the system size very differently than the freeze-out yield of pions and $\eta$ and that the data are compatible with the assumption that they originate from $\Delta$ Dalitz decays, being suggestive of resonance matter [39].

It is the purpose of the present study to investigate this enhancement within the presently available transport codes - HSD, IQMD and UrQMD - in order to explore whether the dilepton production in these systems can be reproduced by the theoretical approaches and to identify eventually the origin of this in-medium enhancement. All these codes have been successfully employed to investigate a multitude of experimental observables. They are, however, not of the same sophistication as far as the dileptons are concerned. The dilepton part of the UrQMD program is still under development and up to now bremsstrahlung is not included. This limits the predictive power to parts of the spectra where bremsstrahlung is not essential. For the study of the in-medium enhancement we limit ourselves to HSD and IQMD calculations. We start out in section II with a short description of the HSD model and of the improvements made compared to the 'standard' HSD 2.5 version used for the extended dilepton study in Ref. [40]. Then we come to a brief description of dilepton production in IQMD. In section III the dilepton production in elementary reactions, measured by the HADES and and DLS collaboration, is compared with HSD and IQMD calculations. The forth section is devoted to the study of dilepton production in heavy-ion collisions. We discuss our calculations for all systems which have been measured by the HADES collaboration and present also our predictions for Au+Au collision at $1.25 \mathrm{AGeV}$ which is presently analysed. After checking that the invariant mass spectra of dileptons in heavy-ion collisions are well reproduced by the HSD as well as by the IQMD approach we study in section V the enhancement of the dilepton production in heavy-ion collisions as compared to the elementary reactions and identify its origin. In section VI we present our conclusions. 


\section{THE TRANSPORT MODELS}

\section{A. The HSD model}

Our analysis of the experimental results is carried out within the off-shell HSD transport model [40, 47 49] based on covariant self energies for the baryons [50]. It has been used for the description of $p A$ and $A A$ collisions from SIS to RHIC energies. We recall that in the HSD approach nucleons, $\Delta^{\prime}$ 's, $\mathrm{N}^{*}(1440), \mathrm{N}^{*}(1535), \Lambda, \Sigma$ and $\Sigma^{*}$ hyperons, $\Xi$ 's, $\Xi^{*}$ 's and $\Omega$ 's as well as their antiparticles are included on the baryonic side whereas the $0^{-}$and $1^{-}$octet states are incorporated in the mesonic sector. Inelastic baryon-baryon (and meson-baryon) collisions with energies above $\sqrt{s_{t h}} \simeq 2.6 \mathrm{GeV}$ (and $\sqrt{s}_{t h} \simeq 2.3 \mathrm{GeV}$ ) are described by the FRITIOF string model [51] whereas low energy hadron-hadron collisions are modelled using experimental cross sections.

The dilepton production by the decay of a (baryonic or mesonic) resonance $R$ can be schematically presented in the following way:

$$
\begin{aligned}
B B \rightarrow & R X \\
m B \rightarrow & R X \\
& R \rightarrow e^{+} e^{-} X, \\
& R \rightarrow m X, m \rightarrow e^{+} e^{-} X, \\
& R \rightarrow R^{\prime} X, R^{\prime} \rightarrow e^{+} e^{-} X .
\end{aligned}
$$

In a first step a resonance $R$ might be produced in baryon-baryon $(B B)$ or meson-baryon $(m B)$ collisions (1), (2). Then this resonance can either couple directly to dileptons (3) (e.g. Dalitz decay of the $\Delta$ resonance: $\Delta \rightarrow e^{+} e^{-} N$ ) or produces mesonic (4) or baryonic (5) resonances which then produce dileptons via direct decays $(\rho, \omega)$ or Dalitz decays $\left(\pi^{0}, \eta, \omega\right)$. With increasing energy hadrons are created by non-resonant mechanisms or string decay. This is also true for those which disintegrate into dileptons. The electromagnetic part of all conventional dilepton sources $\pi^{0}, \eta, \omega, \Delta$ Dalitz decays as well as direct decay of vector mesons $\rho, \omega$ and $\phi$ - are calculated as described in detail in Ref. [54]. We note that we use here again (as in early HSD dilepton studies [47, 48]) the "Wolf" model for the differential electromagnetic decay width of the $\Delta$ resonance [53] instead of the "Ernst" description [30] adopted at that time in [54.

The treatment of the 'enhanced' bremsstrahlung contribution from $p p, p n$ as well as $\pi N$ 'quasi-elastic' scattering, based on the OBE calculations by Kaptari and Kämpfer [41], is discussed in detail in Ref. [40] (cf. Section 2.6 there) where also a discussion of the different models [42 44], which formulate bremsstrahlung in the elementary reactions, can be found. We note here that the OBE models mentioned above 41 44] provide different results not only for the $p N$ bremsstrahlung contribution (which might be attributed to the different way to realize the gauge invariance) but for the $\Delta$-Dalitz decay, due to the different form factors. In our transport analysis we use only the bremsstrahlung contribution from [41] avoiding the uncertainties in the $\Delta$ channel in the OBE models and neglecting the quantum mechanical interference between individual contributions which can not be treated consistently in transport approaches. Also we stress here again that in order to separate the bremsstrahlung $\left(p p \rightarrow p p e^{+} e^{-}\right)$from a vector-dominance like dilepton production via the $\rho$-meson $\left(p p \rightarrow p p \rho, \rho \rightarrow e^{+} e^{-}\right)$, we do not employ a vector-dominance form factor when calculating the bremsstrahlung. Thus, the dilepton radiation via the decay of the virtual photon $\left(p p \rightarrow p p \gamma^{*}, \gamma^{*} \rightarrow e^{+} e^{-}\right)$and the direct $\rho$ decay to $e^{+} e^{-}$are distinguished explicitly in the calculations. In the Section VI we discuss the model uncertainties concerning the treatment of $\Delta$ 's and bremsstrahlung.

The off-shell HSD transport approach incorporates the off-shell propagation for vector mesons as described in Ref. [55] in extension of early BUU transport models [48, 56]. In the off-shell transport description, the hadron spectral functions change dynamically during the propagation through the medium and evolve towards the on-shell spectral functions in the vacuum. As demonstrated in Refs. [20, 40], the off-shell dynamics is important for resonances with a rather long lifetime in the vacuum but strongly decreasing lifetime in the nuclear medium (especially $\omega$ and $\phi$ mesons) and also be proven to be vital for the correct description of the dilepton decay of $\rho$ mesons with masses close to the two pion decay threshold. For a detailed description of the off-shell dynamics and the implementation of in-medium scenarios (as a collisional broadening and/or dropping mass scenario) in HSD as well as for an extension of the LUND string model to include 'modified' spectral functions we refer the reader to Refs. 20, 40, 55].

For the present study we consider the scenario of a 'collisional broadening' of the vector meson spectral functions. This is also supported by experimental data in contrast to the 'dropping mass' scenario (cf. [17 22]). We incorporate the effect of collisional broadening of the vector-meson spectral functions by using for the vector meson width

$$
\Gamma_{V}^{*}\left(M,|\vec{p}|, \rho_{N}\right)=\Gamma_{V}(M)+\Gamma_{\text {coll }}\left(M,|\vec{p}|, \rho_{N}\right) .
$$

Here $\Gamma_{V}(M)$ is the total width of the vector mesons $(V=\rho, \omega)$ in the vacuum. The collisional width in (6) is 
approximated as

$$
\Gamma_{\text {coll }}\left(M,|\vec{p}|, \rho_{N}\right)=\gamma \rho_{N}<v \sigma_{V N}^{t o t}>\approx \alpha_{\text {coll }} \frac{\rho_{N}}{\rho_{0}} .
$$

Here $v=|\vec{p}| / E ; \vec{p}, E$ are the velocity, 3-momentum and energy of the vector meson in the rest frame of the nucleon current and $\gamma^{2}=1 /\left(1-v^{2}\right) ; \rho_{N}$ is the nuclear density and $\sigma_{V N}^{t o t}$ the meson-nucleon total cross section. We use the 'broadening coefficients' $\alpha_{\text {coll }} \approx 150 \mathrm{MeV}$ for the $\rho$ and $\alpha_{\text {coll }} \approx 70 \mathrm{MeV}$ for $\omega$ mesons as obtained in [40]. For the further details we refer the reader to Ref. [40].

We use the time integration method to calculate dilepton spectra which means that vector mesons and resonances can emit dileptons from their production ('birth') up to their absorption ('death'). This is especially important for the study of in-medium effects because this method takes the full in-medium dynamics into account.

We note that it is very important to have an adequate description of the elementary reactions, especially near the threshold where the cross sections grow very rapidly. This rise has a big impact on the description of the experimental data. The comparison of the latest experimental data from the HADES collaboration on $p p$ collisions at $3.5 \mathrm{GeV}$ [57] with HSD calculations shows that the previous parametrizations of $\eta$-meson and of vector mesons $(\rho, \omega)$ production cross sections from Ref. [40] overestimate the data. The over-prediction of the dilepton yield at the $\rho$-peak has been already realized in Ref. [40] from the comparison to the DLS data for $p p$ at $2.09 \mathrm{GeV}$. Thus, we have modified the HSD model accordingly in order to obtain a better description of the existing experimental data on elementary reactions (cf. the discussions in the next subsection).

We note that the HSD model is well tested with respect to the bulk observables at low energy and in light systems, relevant for present study. The pion and eta production from $\mathrm{C}+\mathrm{C}$ collisions at the energies considered here are shown in Section 3 of Ref. [40].

\section{Particle production from elementary reactions}

Here we describe the major changes/improvements made in the HSD model used here compared to the basic HSD version 2.5 used for the dilepton analysis in Ref. [40]:

1) The high energy part of the $p p \rightarrow \eta X$ and the $p n \rightarrow \eta X$ cross sections have newly been parametrized. The

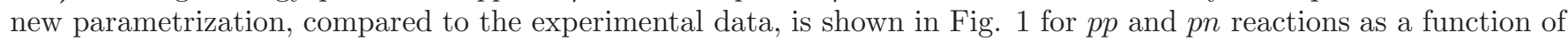
the centre of mass energy above threshold $\left(\sqrt{s}-\sqrt{s_{0}}\right)$. The solid and dashed lines represent the inclusive $p p \rightarrow \eta X$ and $p n \rightarrow \eta X$ cross sections from the HSD model. The experimental data are collected from Refs. 57 61]: the full squares and the open star stand for the exclusive $p p \rightarrow \eta p p$ data, the dots for $p n \rightarrow \eta p n$ data. The full diamond and the full star show inclusive data for $p p \rightarrow \eta X$. The open and full stars indicate the exclusive and inclusive cross sections extrapolated from the dilepton data by the HADES collaboration [57]. It is important to note that in elementary reactions also a deuteron can be produced in the final state via $p n \rightarrow \eta d$. The cross section for this channel is indicated by the dashed-dotted line in Fig. 1, whereas the dotted line shows the $p n \rightarrow \eta X$ cross section including the $p n \rightarrow \eta d$ contribution. The open triangles show the experimental data for $p n \rightarrow \eta d$ from Refs. [58 60]. The channel $p n \rightarrow \eta d$ is not considered in the HSD calculations for $A+A$ and $p+A$ reactions because the probability for deuteron formation in the baryonic medium is negligibly small.

2) The HSD model has also been improved concerning the isospin separation of the vector meson production in baryon-baryon $(B B)$ and secondary meson-baryon $(m B)$ reactions. The isospin averaged cross sections $B B \rightarrow V B B$ $(V=\rho, \omega, \phi)$ and $m B \rightarrow V B$ have been replaced by cross sections which take explicitly the isospin for each channel into account. The new parametrization of the cross section as a function of the centre of mass energy, $\sqrt{s}$ for the $p p$ reaction is compared in Fig. 2 to the experimental data. The solid lines represent the parametrizations of the inclusive $p p \rightarrow V X(V=\rho, \omega)$ cross sections while the dashed lines stand for the exclusive cross sections. We denote these exclusive cross sections for the $\rho$-meson production as 'non-resonant' since in this study we consider explicitly the possible contribution of the baryonic resonance $N(1520)$ to the sub-threshold $\rho^{0}$ production $\left(p p \rightarrow N(1520) p \rightarrow \rho^{0} p p\right)$. It is indicated as the dashed-dotted line on the left plot. The dotted line shows the sum of the inclusive 'non-resonant' and exclusive 'resonant' contribution. The experimental data [59, 63, 64] are shown for exclusive $p p \rightarrow V p p$ (dots) and inclusive $p p \rightarrow V X$ (squares) vector meson production. The stars indicate the inclusive cross sections extrapolated from the dilepton data by the HADES collaboration [57].

We note that we do not propagate explicitly the $N(1520)$ resonance in the HSD approach, rather we consider it as an excitation in the amplitude which enhances the $\rho$-meson production in $N N$ and $\pi N$ reactions at sub-threshold energies. The modelling of the $N(1520)$ production in $N N$ collisions is based on a phase space model with a constant matrix element adopted from Ref. [65]. The contribution of the $N(1520)$ to the $\rho$ cross section is included in line with 


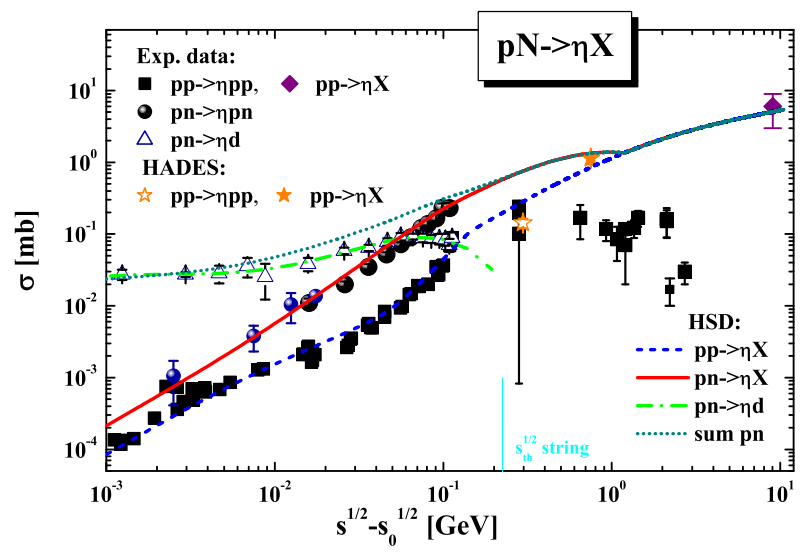

FIG. 1: (Color online) The $\eta$ production cross section in $p p$ and $p n$ reactions as a function of the invariant energy above threshold $\left(\sqrt{s}-\sqrt{s_{0}}\right)$. The solid and dashed lines represent the inclusive $p p \rightarrow \eta X$ and $p n \rightarrow \eta X$ cross sections from the HSD model, the dashed-dotted line indicates the $p n \rightarrow \eta d$ channel and the dotted line the $p n \rightarrow \eta X$ cross section including $p n \rightarrow \eta d$. The experimental data are collected from Refs. 57 61]: the full squares and open star stand for the exclusive $p p \rightarrow \eta p p$ experimental data, the dots for $p n \rightarrow \eta p n$ and open triangles for $p n \rightarrow \eta d$ experimental data; the solid diamond and solid star show inclusive experimental data for $p p \rightarrow \eta X$. The open and full stars indicate the exclusive and inclusive cross sections extrapolated from the dilepton data by the HADES collaboration [57].
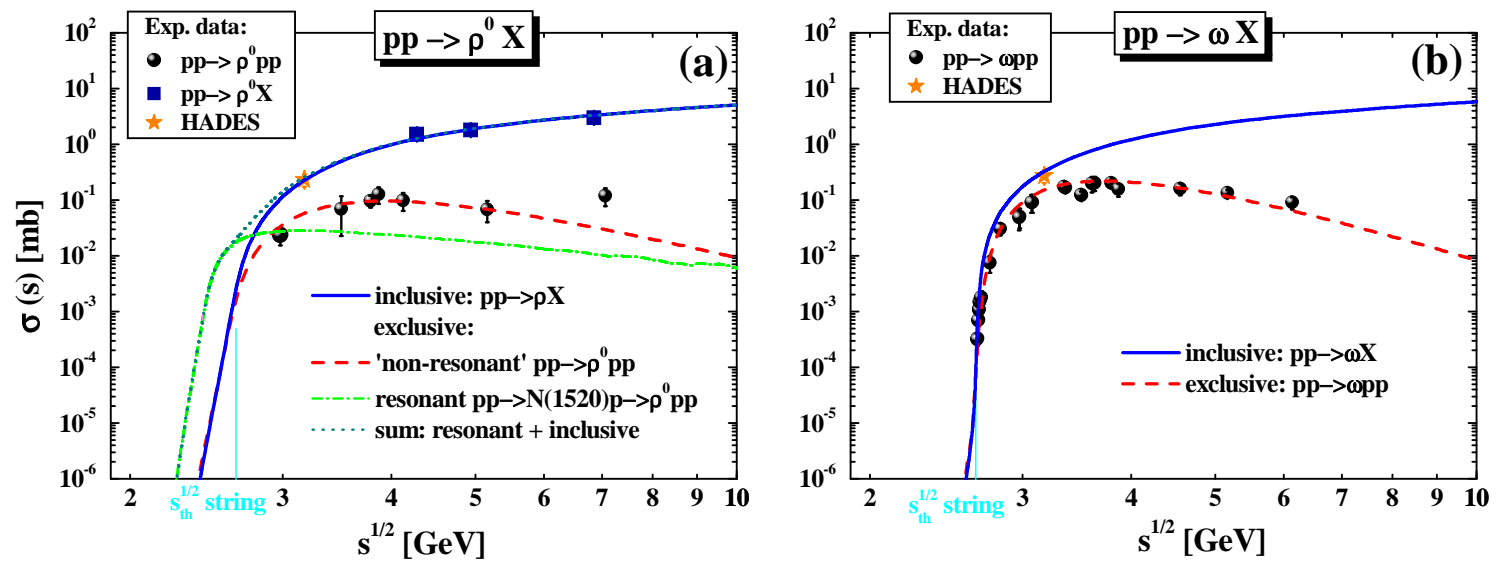

FIG. 2: (Color online) The production cross sections for the channels $p p \rightarrow \rho X$ (left plot (a)) and $p p \rightarrow \omega X$ (right plot (b)) as a function of the centre of mass energy $\sqrt{s}$. The solid lines represent the parametrizations of the inclusive $p p \rightarrow V X(V=\rho, \omega)$ cross sections while the dashed lines stand for the exclusive 'non-resonant' cross sections. The dashed-dotted line on the left plot shows the contribution from the $N(1520)$ resonance to the $\rho^{0}$ production via the process $p p \rightarrow N(1520) p \rightarrow \rho^{0} p p$ and the dotted line indicates the sum of the inclusive non-resonant and exclusive resonant contributions. The experimental data [59, 63, 64] are shown for exclusive $p p \rightarrow V p p$ (dots) and inclusive $p p \rightarrow V X$ (squares) vector meson production. The stars indicate the inclusive cross sections extrapolated from the dilepton data by the HADES collaboration [57]. The vertical light blue lines show the threshold for meson production by string formation and decay $\left(\sqrt{s}_{t h}=2.6 \mathrm{GeV}\right)$ as implemented in HSD for baryon-baryon channels.

Ref. [1] which has been used in our previous work [33]. The decay channels of the $N(1520)$ resonance are not well established. Especially the disintegration into a $\rho$ is estimated in between $15-25 \%$. Including this contribution from the $N(1520)$ resonance decay presents an upper estimate for the $\rho$-meson production in $N N$ and $\pi N$ reactions at sub-threshold energies. This model assumption can be checked experimentally via an observation of an enhancement of the dilepton yield near the $\rho$-peak in the elementary reactions at sub-threshold energies. In the case of heavy-ion collisions at low bombarding energies the contribution of the $N(1520)$ resonance to the dilepton spectra can hardly be seen especially not in reactions of the light nuclei as $\mathrm{C}+\mathrm{C}$ as measured by the HADES collaboration at 1.0 $A \mathrm{GeV}$. The Fermi motion modifies the available energy for meson production and due to the rapid rise of the cross section at threshold the inclusive $\rho$-meson production mechanism starts to dominate, see Fig. 2] even if the nominal 
energy is below threshold. On the other hand, the $N(1520)$ resonance can be excited by pion-baryon collisions and contribute to the $\rho$-meson production via the process $\pi N \rightarrow N(1520) \rightarrow \rho N$. The probability of such processes is larger for heavy nuclei collisions but the pion density is relatively small at sub-threshold energies where the possible contribution of $N(1520)$ plays a role. Consequently, the enhancement of the $\rho$-meson production by accounting for the $N(1520)$ channel is relatively small in the HSD model. This differs from e.g. the UrQMD model [71, 72] where a much larger cross sections for the $N(1520)$ production is used. We will come back to this discussion in Section IV.

3) We improved also the description of multi-meson production between the two pion production threshold and $\sqrt{s}=2.6 \mathrm{GeV}$ where we match the standard HSD description of particle production via strings.

Close to the two-pion threshold the two pions are dominantly produced by the decay of 2 Deltas created in NN collisions. With increasing energy the available phase space is sufficient for multi-meson production and a lot of extra channels become open. However, it is unknown whether the light mesons are produced by the decay of heavy baryonic resonances or directly from the excitation and decay of the strings. Since there is very little experimental information on the exclusive channel decomposition in this 'intermediate' energy range we used the FRITIOF LUND string model as an 'event generator' for the production of such 'multi-meson' channels by adding them to the exclusive channels which are modeled in the HSD explicitly, such that we obtain the inelastic $N N$ cross section, i.e. $\sigma_{\text {inel }}=\sigma_{\text {excl }}+\Delta \sigma_{\text {incl }}$, where $\sigma_{e x c l}$ stands for the exclusive channels such as $N N \rightarrow \Delta N, N N \rightarrow \Delta N m, m \equiv \pi, \rho, \omega, \phi, \ldots$ and channels with strangeness production such as $N N \rightarrow Y N K(Y \equiv \Lambda, \Sigma)$ and $N N \rightarrow N N K \bar{K}$. Here $\Delta \sigma_{\text {incl }}$ corresponds to the sum of the 2 pion production channels as $N N \rightarrow \Delta \Delta$ and 'multi-meson' production $N N \rightarrow N N(\Delta)+n \times m$ $(n=2,3,4, \ldots)$ and channels with the final hyperons and strange mesons. We note that since close to the threshold the FRITIOF model doesn't provide the correct isospin decomposition for $\Delta$ production, since e.g. an exclusive channel $N N \rightarrow \Delta^{++} n$ is missing, we have adjusted the FRITIOF model to correct for the isospin decomposition of produced $\Delta$ 's: for the exclusive channel $p p \rightarrow N \Delta$ we assume now that $3 / 4$ of the produced $\Delta$ are in the $\Delta^{++}$state and only $1 / 4$ in the dilepton producing $\Delta^{+}$state. This leads to a reduction of $\Delta^{+}$production and an enhancement of $\Delta^{++}$ production, respectively.

The excitation function of the multiplicity of the different pions in HSD is shown in Fig 3 , on the left hand side for $p p$ collisions, on the right hand side for $p n$ reactions. These multiplicities are compared with the available data which are very scarce for $p n$ reactions. Additionally to the total pion multiplicity, the multiplicity of $\Delta$ 's themself is very important for the dilepton study because the $\Delta$ resonances decay into pions as well as into dileptons whereas other sources of pions do not contribute to the dilepton yield. The $\Delta$ production in $p p$ collisions in the HSD approach is shown in Fig. 4 and compared with the available experimental data. Here the production cross sections for the inclusive channels $p p \rightarrow \Delta^{+} X$ (solid line) and for the exclusive channel $p p \rightarrow \Delta^{+} p$ (dashed line) from HSD are presented as a function of the invariant energy $\sqrt{s}$. The experimental data [63] are shown for exclusive $p p \rightarrow \Delta^{+} p$ production. The star indicates the extrapolation for the $\Delta$ inclusive cross section from the dilepton spectra by the HADES collaboration based on the PLUTO simulation program [52] from Ref. [57]. One can see from Fig. 4 that the inclusive $\Delta$ production dominates the exclusive one already at relatively low $\sqrt{s}$. However, due to the lack of inclusive experimental data on $\Delta$ production it is hard to justify the modeling of $\Delta$ dynamics beyond the exclusive channels which are relatively well known experimentally and accurately modeled in transport approaches.

As said, above a kinetic energy of $1.5 \mathrm{GeV}$ there is no experimental information available on whether resonance are involved in the production. This is the reason way different parameterizations have been advanced. For example in the resonance based GiBUU model [82] a lower inclusive $\Delta^{+}$production cross section is used as compared to HSD. Also the isospin relations are different which leads to a lower dilepton contribution from $\Delta$ Dalitz decay. Additionally the different parametrization for the differential dilepton decay width is employed which lowering the $\Delta$ Dalitz decay channal substantially compare to the HSD (cf. discussions in Section VI).

\section{B. Open questions related to the elementary reactions in transport models}

In nucleon-nucleon collisions at low energies, i.e. below $\sqrt{s}<2.2 \mathrm{GeV}$, very seldom more than one meson is produced. The cross sections for these reactions have been measured experimentally (cf. [59]) and are used in the transport approach. Above $\sqrt{s} \approx 2.2 \mathrm{GeV}$ the multi-meson production starts to dominate, but the experimental information on inclusive as well as exclusive multi-meson production channels are very poor. Also it is not known whether the mesons are directly produced or whether they are decay products of intermediate resonances or strings. The theoretical analysis of these data has not produced yet a consistent knowledge on the channel decomposition [66 69]. This introduces large uncertainties for the prediction of the dilepton yield in transport theories because it depends on the formation of specific intermediate resonances. We note that in the UrQMD model the production of mesons at intermediate energies is realized exclusively via excitation and decay of heavy baryonic resonances which 

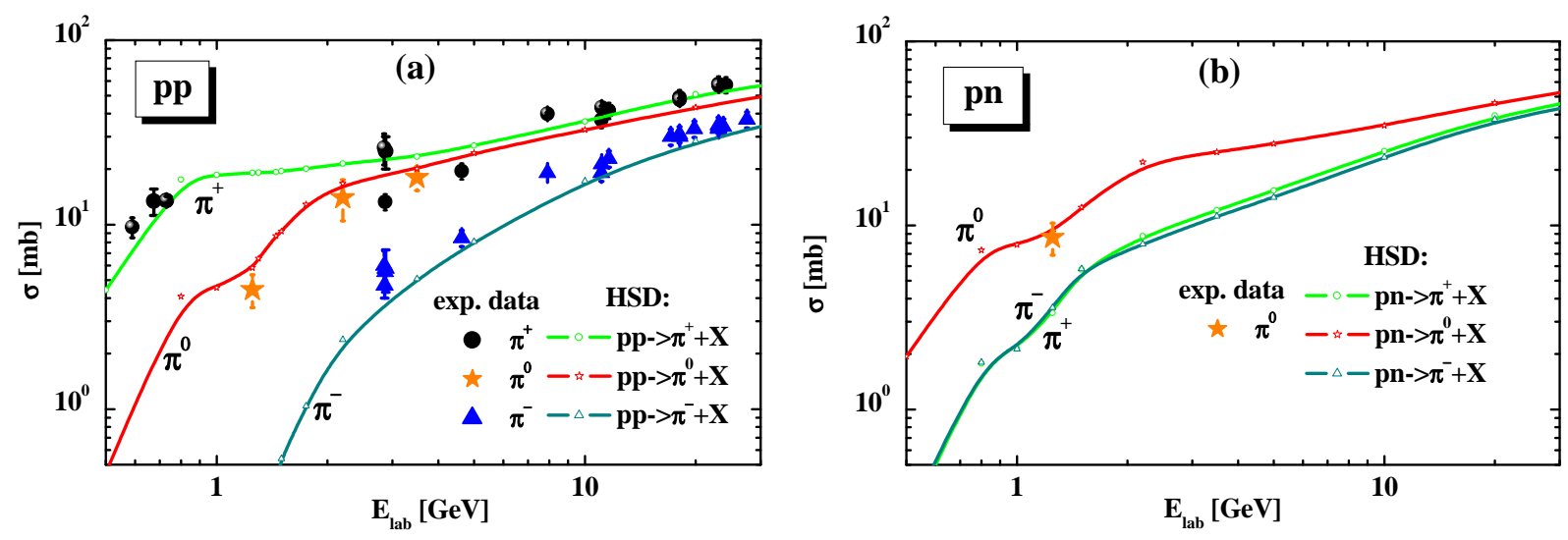

FIG. 3: (Color online) Left (a): The inclusive pion production cross sections as a function of the proton bombarding energy $E_{l a b}$. The HSD results are shown in terms of lines with open symbols whereas the experimental data are indicated by the corresponding solid symbols, i.e. for $p p \rightarrow \pi^{+}+X$ : HSD - the solid line with open dots, experimental data - solid dots from Refs. 63, 78]; for $p p \rightarrow \pi^{0}+X$ : HSD - the solid line with open stars, the HADES data - full stars from Refs. [57, 62]; for $p p \rightarrow \pi^{-}+X:$ HSD - the solid line with open triangles, experimental data - full triangles from Refs. [63, 78]. Right (b): The production cross sections for $p n \rightarrow \pi X, \pi=\pi^{+}, \pi^{0}, \pi^{-}$from the HSD model, the HADES data - full star from the extrapolation in Ref. [37].

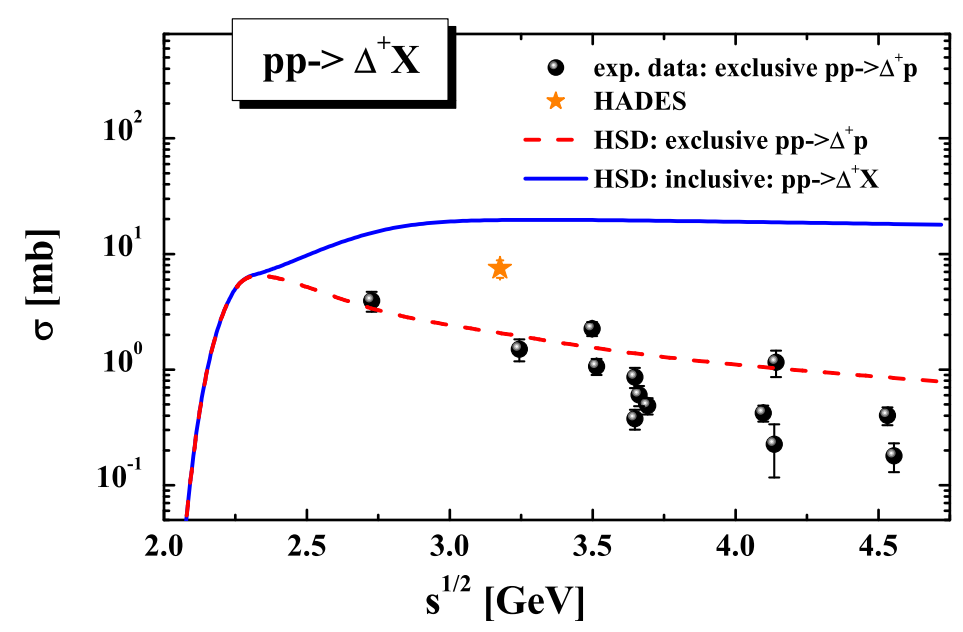

FIG. 4: (Color online) The production cross sections for the inclusive channels $p p \rightarrow \Delta^{+} X$ (solid line) and the exclusive channel $p p \rightarrow \Delta^{+} p$ (dashed line) from HSD as a function of the invariant energy $\sqrt{s}$. The experimental data 63 are shown for exclusive $p p \rightarrow \Delta^{+} p$ production. The star indicates the inclusive cross section extrapolated from the dilepton data by the HADES collaboration [57].

are explicitly propagated in the transport model 71, 72], whereas in HSD the string mechanism is used (as discussed above) for the description of the same final meson spectra. Thus, one needs more exclusive experimental information in order to differentiate between the models.

\section{The IQMD model}

The IQMD model used for the calculations in this study is the same as introduced in the first IQMD paper on dilepton production [45]. In this model all pions are produced by the decay of $\Delta$ resonances. Because no higher mass resonances are included we limit the prediction to beam energies up to $2 A \mathrm{GeV}$. The excitation function of the pion yield for the $\mathrm{Ca}+\mathrm{Ca}$ system, compared with the available data, is shown in Fig. 5. We see that the pion multiplicity, the result of a complicated interplay between $\Delta$ creation, absorption and decay, is quite reasonable reproduced by the IQMD approach [76]. This is also the case for heavier systems [77. Thus both, the IQMD as well as the HSD approach, describe the available pion data quite well, a prerequisite for an analysis of the dilepton spectra which are 
not only normalized to the pion yield but have an important contribution from the $\Delta$ decay. For other models which are used to describe the dilepton production, like [70], it remains to be seen whether they reproduce heavy-ion pion data.

For the calculations of the dilepton spectra the standard IQMD program [73, 74] has been supplemented with all elementary cross sections which are important for this process 45]. For that we have used the parametrizations of available experimental data, but for many channels, $p p$ data are only available for low $\sqrt{s}$ values and $n p$ data are very scarce. Consequently, in heavy-ion collisions at beam energies larger than $1.5 \mathrm{AGeV}$ most of the particles which emit dileptons are produced using theoretically calculated cross sections. In Ref. [45] we have studied how the uncertainties of the cross sections from elementary reactions influence the dilepton spectra in heavy-ion collisions. For these studies we use the set up in which the $p n \rightarrow \omega p n$ cross section is 5 times higher than the $p p \rightarrow \omega p p$ cross section. This explains the difference between HSD and IQMD at dileption invariant masses close to the $\rho, \omega$ peak.

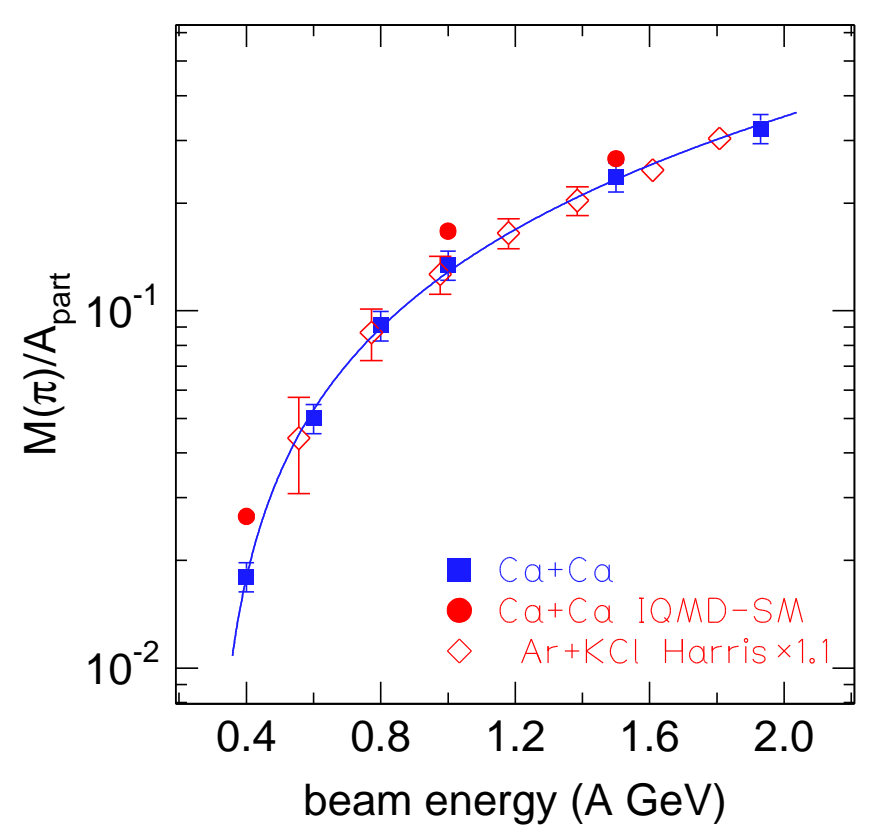

FIG. 5: (Color online) The excitation function of the $\pi$ multiplicity per participating nucleon $\left(\left(N\left(\pi^{+}\right)+N\left(\pi^{-}\right)\right) / A_{\text {part }}\right)$ for $\mathrm{Ca}+\mathrm{Ca}$ collisions using $A_{\text {part }}$ as $0.9 \mathrm{~A}$. Data of the FOPI collaboration are compared with data of Harris et al. 75] and predictions of the IQMD model [76, 77].

In the IQMD approach the dileptons are calculated perturbatively using the 'spontaneous decay' method - contrary to the time integration method in HSD and UrQMD. It is based on the assumption that all hadrons which decay into dileptons and which are produced in the heavy-ion collision contribute to the dilepton yield as if they were produced in free space. This implies that a possible later reabsorption of the hadrons is not taken into account. Because in reality some of the $\Delta$ 's and of the other dilepton producing hadrons are reabsorbed, the IQMD calculations give an upper limit for the dilepton production in heavy-ion collisions. Consequently, the spontaneous decay method limits the approach to small systems contrary to the time integration method which follows the in-medium dynamics of all dilepton sources exactly. However, for the systems studied here the 'spontaneous decay' method is still acceptable. For the details of the cross sections for the creation of dilepton producing particles we refer to Ref. [45].

\section{DILEPTON PRODUCTION IN ELEMENTARY $p p, p d$ AND $p+A$ REACTIONS}

The first reaction considered here is the dilepton production in elementary reactions like $p+p$, quasi-free $p+n(d)$ and $p+N b$ reactions. 


\section{A. Dilepton production in pp and pd at energies around $1.25 \mathrm{GeV}$}

We start our discussion with the HADES and DLS data a $1.25 \mathrm{GeV}$. Fig. 66 shows the differential cross section $d \sigma / d M$ for dileptons as a function of the invariant mass $\mathrm{M}$ for $p p$ (left), $p n$ (middle) and $p d$ (right) reactions at $1.25 \mathrm{GeV}$. The HSD results are presented in comparison to the experimental data from the HADES collaboration 37, 38]. The different lines display the contributions from the various channels in the HSD calculations (for the colour coding we refer to the legend). We note here (and that applies to all further plots) that the theoretical calculations passed through the appropriate experimental acceptance filters and that the mass/momentum resolution is taken into account.

As seen in the left part of Fig. 6 the $p p$ dilepton yield is dominated by the $\Delta$-Dalitz decay while bremsstrahlung is sub-leading due to the destructive interference between initial and final state amplitudes in case of equal charges due to a different sign in the acceleration. Thus these HADES data provide a solid constraint on the $\Delta$ production whose control will be very important for a robust interpretation of the heavy-ion data. In $p n$ collisions, however, bremsstrahlung is dominating as can be seen from the middle part of Fig. [6. Because the form of the dilepton invariant mass spectrum from $\Delta$ decay and from bremsstrahlung is not completely the same, the form of the $p p$ and the $p n$ spectra is not identical and we see in $n p$ a slight enhancement close to the kinematic limit. In the right part of Fig. [6 we compare the HSD results for $p d$ collisions with the so called quasi-free $p n$ HADES data, used later as the 'reference' spectra $N N=(p n+p p) / 2$ for the interpretation of the heavy-ion data. Experimentally the quasi-free $p n$ events have been separated by measuring the proton spectator in the $p d$ reactions at $1.6<p_{l a b}<2.6 \mathrm{GeV} / \mathrm{c}$.

The comparison of the $p p$ and the $p n(d)$ data shows clearly that in $p n(d)$ the proton does not scatter on a quasi free neutron. The kinematical limit for the invariant mass of the dilepton which is $M_{\max }=\sqrt{s_{N N}}-2 m_{N}=0.545$ $\mathrm{GeV}$ in $p p$ and $n p$ collisions is well exceeded in the $p d$ collisions. The largest invariant mass observed $(M \approx 0.66$ $\mathrm{GeV}$ ) corresponds to the maximal invariant mass which is kinematically allowed in the three body pd system under the condition that the outgoing proton has at least a momentum of $1.6 \mathrm{GeV} / \mathrm{c}$. Therefore at the upper end of the invariant mass spectra we have a collision of the proton with the deuteron with a center of mass energy of $\sqrt{s_{p d}}=\sqrt{\left(p_{p}+p_{d}\right)^{2}}$. This observation one has to keep in mind for the interpretation of dilepton production in heavy-ion collisions, when the $p d$ results are used as a reference to discuss the in-medium enhancement of the dilepton yield.

In semiclassical transport calculations, like HSD, one simulates the deuteron as a bound system of a proton and a neutron which are redistributed in coordinate and momentum space according to the wave function of the Paris potential [80]. The energy of each nucleon (in the deuteron rest frame) is taken as $E_{N}=m_{N}+\varepsilon / 2$, where $\varepsilon=-2.2 \mathrm{MeV}$ is the binding energy of the deuteron. We use the energy-momentum relation for free particles to determine the effective mass of the nucleon and then the energy-momentum 4-vector to describe the collision. An incoming nucleon scatters with one or subsequently with both nucleons of the deuteron but never with the two at the same time. This gives another kinematics as compared to a true three-body collision and therefore HSD calculations underpredict the dilepton production close to the kinematical limit of $p d$ collisions.

Another problem with the quasi-free $p n$ scattering is related to the possibility of deuteron formation in the final state. This is not probable in heavy-ion collisions (cf. Ref. [81]) and not included in HSD. However, as seen from the Fig. 11, the process $p n \rightarrow \eta d$ might be important for the $\eta$-production at threshold energies. Thus, we include this contribution as an enhanced cross section for $\eta$ production in $p n$ (this was not included in our previous work 40]) but we do not treat the deuteron formation explicitly in the code. As seen from the right part of Fig. 6 in np collisions around $M=0.4 \mathrm{GeV}$ the $\eta$ contribution turns out to be of the same order of importance as $\Delta$-Dalitz decays and bremsstrahlung.

Figure 6 (right) shows that in $p d$ collisions the HSD model underestimates the dilepton yield between $0.35<$ $M<0.5 \mathrm{GeV}$, a region which is accessible in two-body collisions at this energy. A possible candidate to explain this enhancement is the contribution of sub-threshold $\rho$-meson production via excitation and decay of the $N(1520)$ resonance shown as the dashed-dotted line in Fig. 2. A very small contribution of this resonant $\rho$ production channel is even seen in $p p$ collisions (dotted line on the left plot). However, this contribution is not sufficient to describe the experimental data. This is in line with a recent study by the GiBUU group [82]. Also IQMD calculations fail to describe this part of the spectrum.

Fig. 7 shows the IQMD predictions for $p p$ and $n p$ collisions as well compared to $p p$ and $p d$ HADES data. We see a very good agreement between HSD and IQMD predictions for the elementary $p p$ and $p n$ reactions.

The cross section $d \sigma / d M$ at $1.27 \mathrm{GeV}$, calculated in the HSD model, is compared in Fig. 8 to the $p p$ (left) and $p d$ (right) DLS data [24] . The theoretical calculations passed through the corresponding DLS acceptance filter and mass resolution. While the agreement between HSD and the data looks reasonable, one has to keep in mind that due to the very broad mass resolution the spectra are strongly distorted at large invariant masses. There seems to be an underestimation of the last experimental point for $p d$, however, the quality of the data does not allow for robust conclusions. 

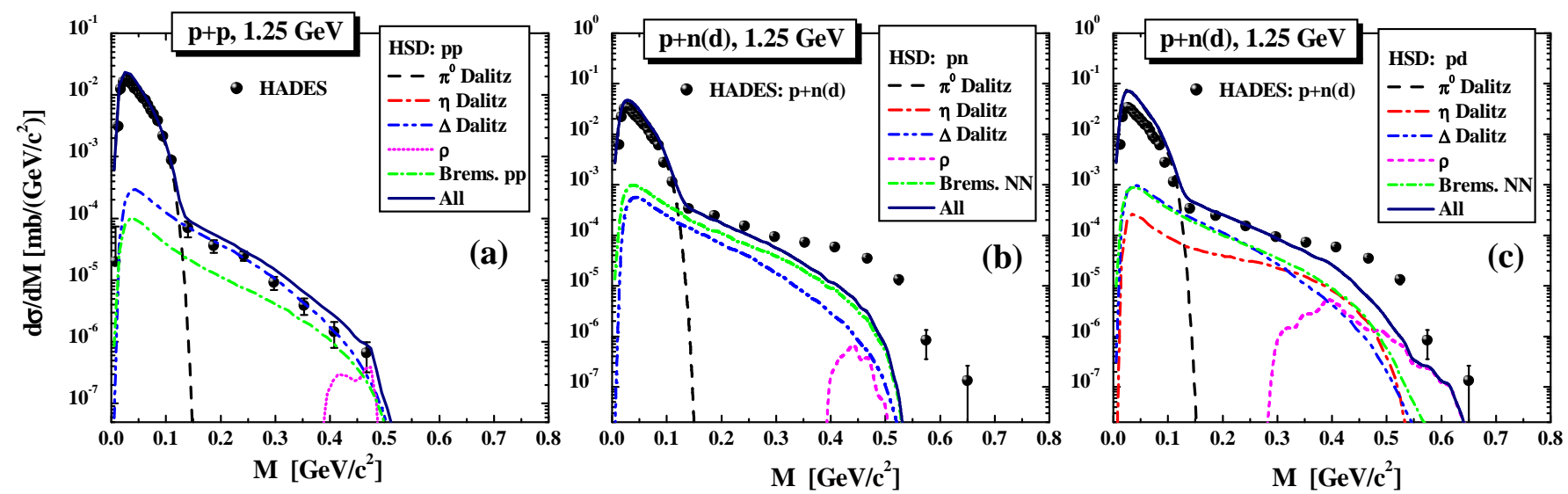

FIG. 6: (Color online) The HSD results for the dilepton differential cross section $d \sigma / d M$ for $p p$ (left plot (a)), $p n$ (middle plot (b)) and $p d$ (right plot (c)) reactions at $1.25 \mathrm{GeV}$ in comparison to the experimental data for $p p$ (left) and quasi-free $p n$ (middle and right plots) reactions from the HADES collaboration [37, 38]. The individual colored lines display the contributions from the various channels in the HSD calculations (see color coding in the legend). The theoretical calculations passed through the corresponding HADES acceptance filters and mass/momentum resolutions.
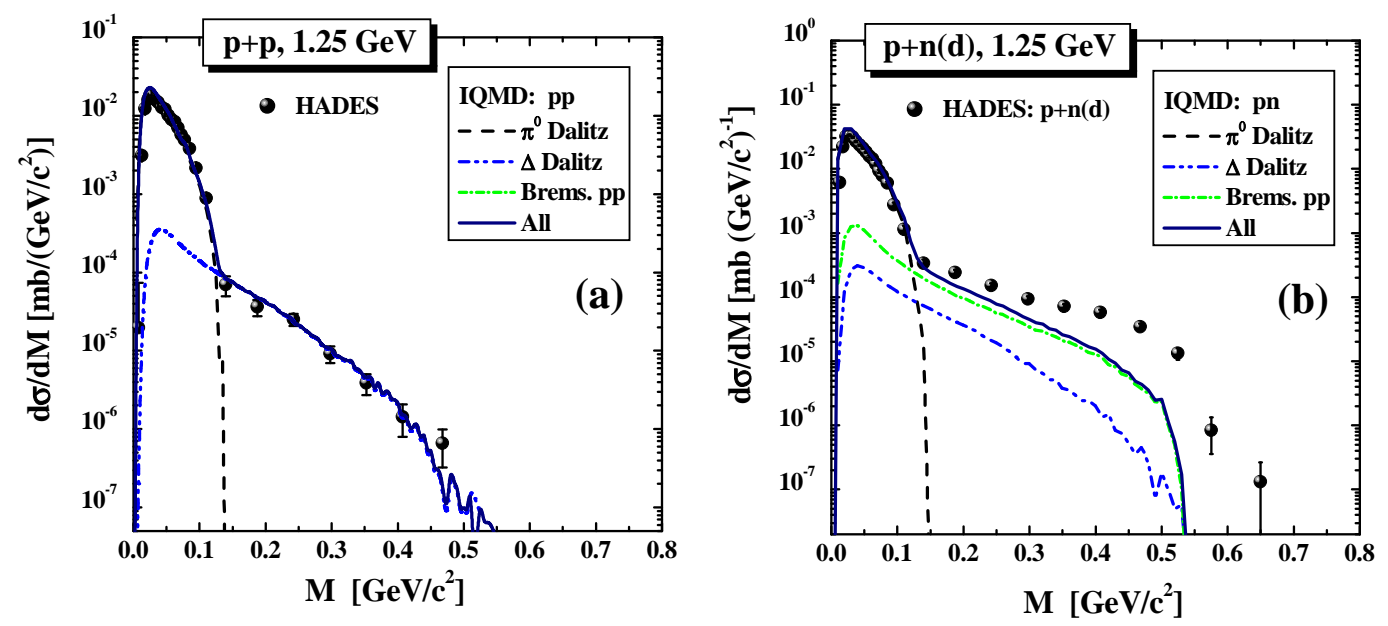

FIG. 7: (Color online) The IQMD results for the dilepton differential cross section $d \sigma / d M$ for $p p$ (left (a)) and $p n$ (right (b)) reactions at $1.25 \mathrm{GeV}$ in comparison to the experimental data for $p p$ (left) and quasi-free $p n(d)$ (right) reactions from the HADES collaboration [37, 38]. The individual colored lines display the contributions from the various channels in the IQMD calculations (see color coding in the legend). The theoretical calculations passed through the corresponding HADES acceptance filters and mass/momentum resolutions.

\section{B. Dilepton production in pp and pd at energies around $2.2 \mathrm{GeV}$}

The differential cross section $d \sigma / d M$ from HSD calculations for $e^{+} e^{-}$production in $p p$ reactions at bombarding energies of $2.2 \mathrm{GeV}$ in comparison to the HADES data 62] is presented in Fig. 9 (left). The right part of Fig. 9 shows for the same reaction the HSD results for the differential transverse momentum cross sections for $p p$ at $2.2 \mathrm{GeV}$ separated for different invariant mass bins: $M \leq 0.15 \mathrm{GeV}, 0.15 \leq M \leq 0.55 \mathrm{GeV}$ and $M \geq 0.55 \mathrm{GeV}$. Also at an energy of $2.2 \mathrm{GeV}$ we see a quite satisfying agreement between theory and experiment.

Fig. 10 shows the dilepton differential cross section $d \sigma / d M$ for the $p p$ (left plot) and $p d$ (right plot) at $2.09 \mathrm{GeV}$ from HSD calculations in comparison to the DLS data 24]. We see also here a good agreement and the fact that the DLS as well as the HADES data are reproduced with the same theory underlines the consistency of both data sets which have quite different acceptance cuts. 

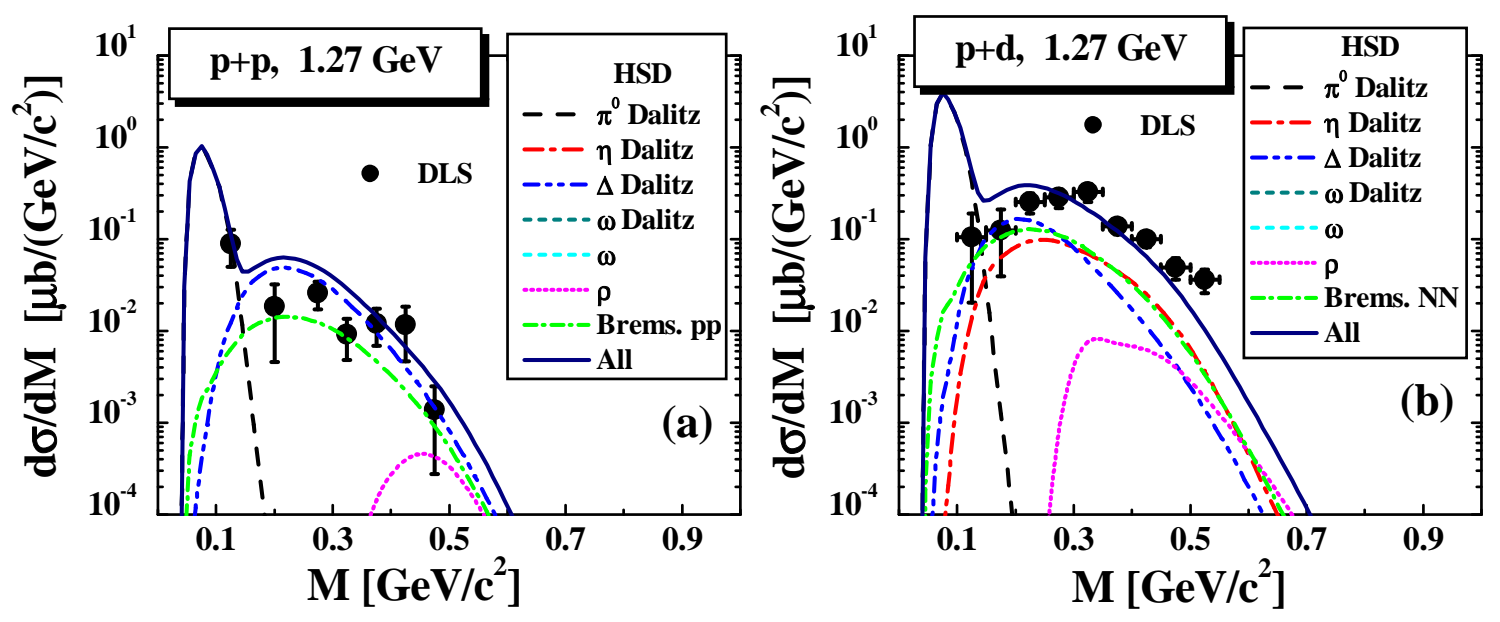

FIG. 8: (Color online) The dilepton differential cross section $d \sigma / d M$ for $p p$ (left plot (a)) and $p d$ (right plot (b)) at 1.27 GeV in comparison to the DLS data 24]. The HSD calculations passed through the corresponding DLS acceptance filter and mass resolution.
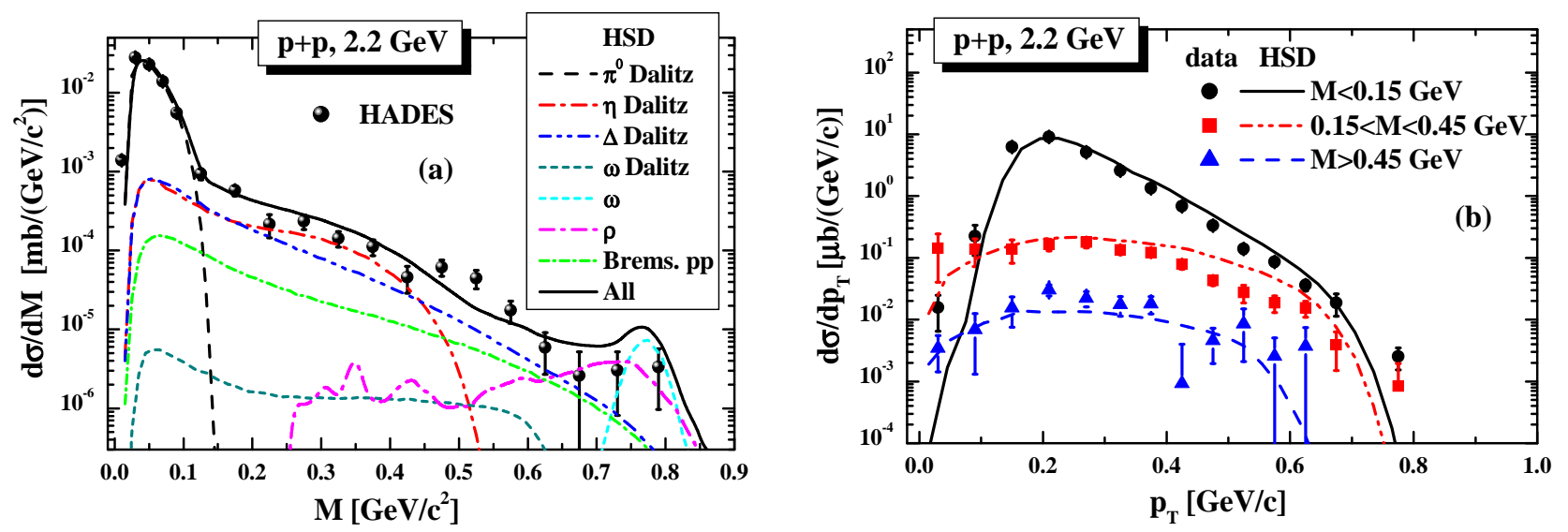

FIG. 9: (Color online) Left (a): the differential cross section $d \sigma / d M$ from HSD calculations for $e^{+} e^{-}$production in $p p$ reactions at a bombarding energy of $2.2 \mathrm{GeV}$ in comparison to the HADES data 62. The individual coloured lines display the contributions from the various channels in the HSD calculations (for the colour coding see legend). Right (b): HSD results for the differential dilepton transverse momentum cross section for $p p$ at $2.2 \mathrm{GeV}$ and for different mass bins: $M \leq 0.15 \mathrm{GeV}$, $0.15 \leq M \leq 0.55 \mathrm{GeV}$ and $M \geq 0.55 \mathrm{GeV}$. The theoretical calculations passed through the corresponding HADES acceptance filter and mass/momentum resolution.

\section{Dilepton production in pp at $3.5 \mathrm{GeV}$}

Finally we come to the HADES pp data at $3.5 \mathrm{GeV}$. Although HADES has not measured heavy ion collisions at this energy we include these results for completeness. Fig. 11] shows the differential cross section $d \sigma / d M$ from HSD calculations for dilepton production in $p p$ reactions at a bombarding energy of $3.5 \mathrm{GeV}$ in comparison to the HADES data [57]. We present the results including and excluding the bremsstrahlung contribution because at this energy there exist no solid bremsstrahlung calculations. The validity of our approach, to take the Kaptari and Kaempfer matrix element and to adjust only the phase space, as described in detail in Ref. [40], becomes questionable at such a high energy. The thick lines, labelled in the legend as "All wo Brems", show the sum of all channels (labelled as "All") without $p p$ bremsstrahlung. For the distribution of the invariant masses of the dileptons, bremsstrahlung does not play a major role at this energy in $p p$, as expected.

In Fig. 12 we compare the HSD results for $p p$ at $3.5 \mathrm{GeV}$ and for 4 different mass bins: $M \leq 0.15 \mathrm{GeV}, 0.15 \leq M \leq$ $0.47 \mathrm{GeV}, 0.47 \leq M \leq 0.7 \mathrm{GeV}$ and $M \geq 0.7 \mathrm{GeV}$ to the HADES data [57]. The upper 4 plots show the rapidity distribution and the lower 4 plots the transverse momentum spectra. As in Fig. 11 the thick lines, labelled in the legend as "All wo Brems", show the sum of all channels (labelled as "All") without pp bremsstrahlung. We observe that the rapidity distribution is well described except for invariant masses around the $\rho$ peak where we overpredict the 

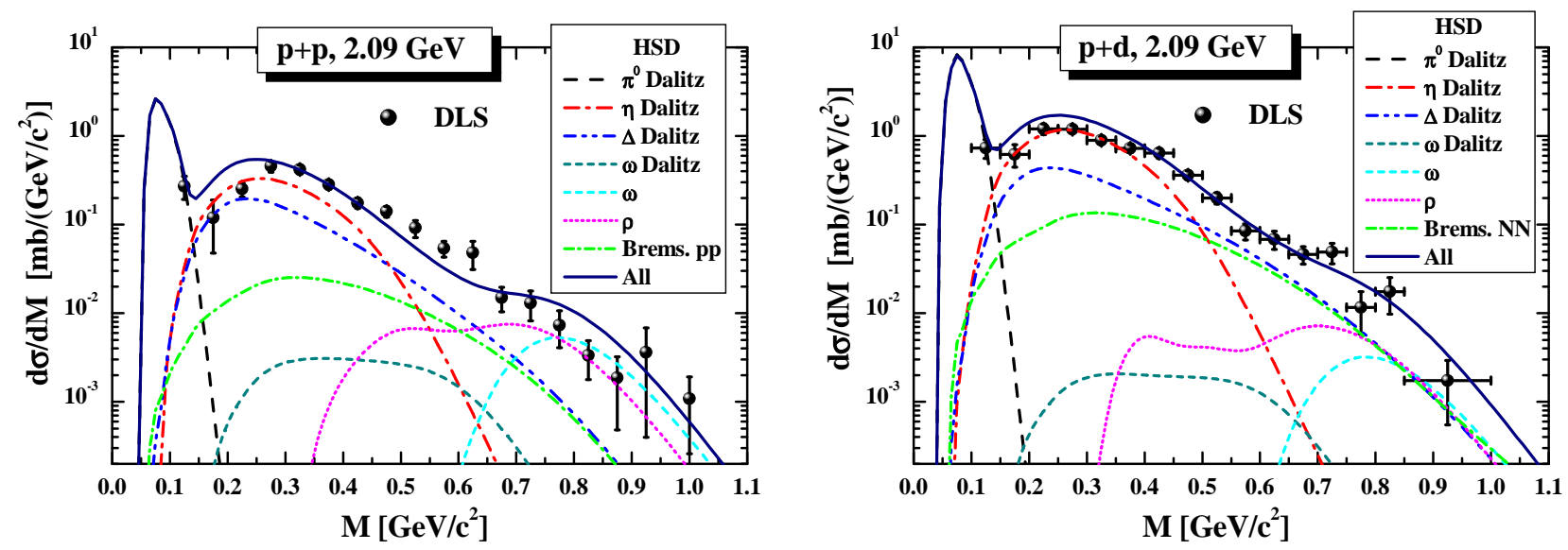

FIG. 10: (Color online) The dilepton differential cross section $d \sigma / d M$ for the $p p$ (left plot (a)) and $p d$ (right plot (b)) at 2.09 $\mathrm{GeV}$ in comparison to the DLS data 24]. The theoretical calculations passed through the corresponding acceptance filters and mass resolutions.

data by a constant factor. Also the transverse momentum distribution is well described by theory with the exception of a region around $M \approx 0.6 \mathrm{GeV}$ where our calculations overpredict the data.

We note that the present result is in a better agreement with the HADES $p_{T}$ data as compared to the early HSD predictions [40, 57] due to the following reasons: a lowering of the $\eta$ Dalitz dilepton contribution due to the reduction of the $\eta$ production cross section in line with the new HADES data (cf. discussion in Section II.A.1(1)); a lowering of the direct $\rho, \omega$ dilepton decay contributions due to the modification of the vector meson production cross section (cf. discussion in Section II.A.1(2)); a lowering of the $\Delta$ Dalitz dilepton contribution due to the adjustment of the isospin decomposition in the exclusive channel $N N \rightarrow \Delta^{++} n$ from FRITIOF (cf. discussion in Section II.A.1 (3)). The latter reduces the total (inclusive) $\Delta^{+}$production by a factor up to 1.4 at $3.5 \mathrm{GeV}$ and correspondingly the dilepton yield. This reduction is even larger (more then a factor of 3 ) for dileptons with high invariant masses and high $p_{T}$ since they stem dominantly from the Dalitz decay of exclusive $\Delta$ 's simply due to kinematical reasons - a lower amount of associated particles leaves more energy for the generation of high mass $\Delta$ 's. An addition reduction of the $\Delta$ dilepton yield stems from the different parametrizations used for the differential electromagnetic decay width of the $\Delta$ resonance (cf. discussion in Section VI): presently - "Wolf" 53 instead of the original "Ernst" description [30] with a coupling constant $g=3$ instead of $g=2.7$ which is consistent with the 'photon' $(M \rightarrow 0)$ limit. Without these modification the present HSD version reproduces the results of [40, 57].

We speculate that HSD produces slightly too many $\Delta$ at $3.5 \mathrm{GeV}$. Since the elementary cross section for inclusive $\Delta$ production in $p p$ reactions at this energy is not available, the repartition of the pion yield between $\Delta$ resonances (which produce dileptons) and other resonances (which do not produce dileptons) is not well known and may be the origin of the deviation obtained in the $p_{T}$ spectra. For the mass bin $0.47<M<0.7 \mathrm{GeV}$ we see that above $p_{T}=0.7$ $\mathrm{GeV} / c$ bremsstrahlung is the dominating source of dilepton production. We plot the sum of all contributions without bremsstrahlung as well.

\section{Dilepton production in pA collisions at $3.5 \mathrm{GeV}$}

We are coming now to $p+A$ reactions. Fig. 13 compares the differential cross section $d \sigma / d M$ from HSD calculations for $e^{+} e^{-}$production in $p+N b$ reaction at a bombarding energy of $3.5 \mathrm{GeV}$ to the HADES data [79]. The upper part shows the case of the 'free' vector-meson spectral functions while the lower part gives the result for the 'collisional broadening' scenario. Again the thick lines, labeled in the legend as "All wo Brems", show the sum of all channels (labeled as "All") without $N N$ bremsstrahlung. We display both cases since the treatment of bremsstrahlung using the extrapolation of the OBE model to such high energy is questionable, as discussed above. For the same reason the $\pi N$ bremsstrahlung presented Fig. in 13 has to be considered with care. The collisional broadening scenario comes closer to the experimental results in the region around the $\rho$ peak. We thus find a nice agreement between theory and experiment also for proton-nucleus collisions. 


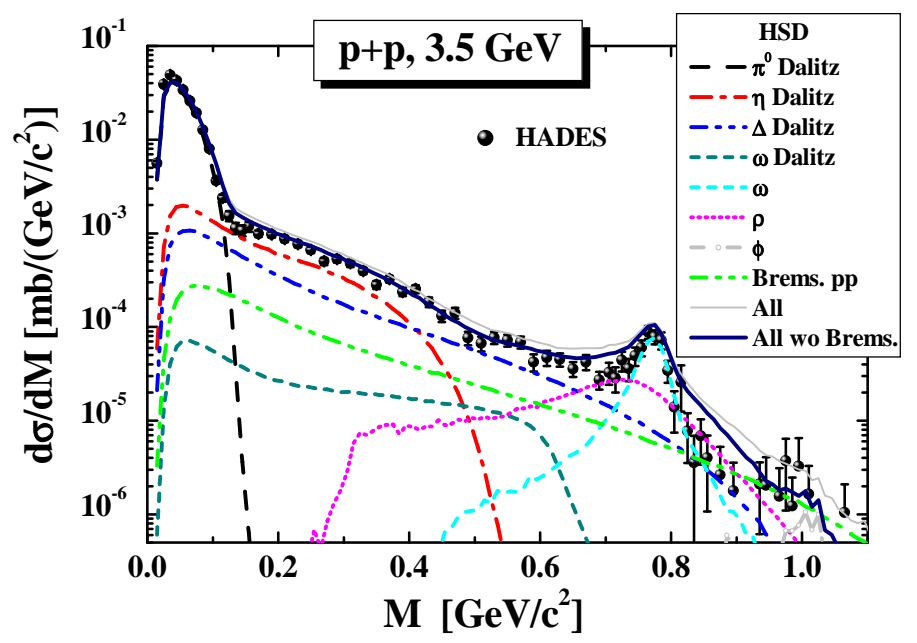

FIG. 11: (Color online) The differential cross section $d \sigma / d M$ from HSD calculations for $e^{+} e^{-}$production in $p p$ reactions at a bombarding energy of $3.5 \mathrm{GeV}$ in comparison to the HADES data [57]. The individual colored lines display the contributions from the various channels in the HSD calculations (see color coding in the legend). The thick line, labeled as "All wo Brems", shows the total sum of all channels (labeled as "All") without $p p$ Bremsstrahlung. The theoretical calculations passed through the corresponding HADES acceptance filters and mass/momentum resolutions. 

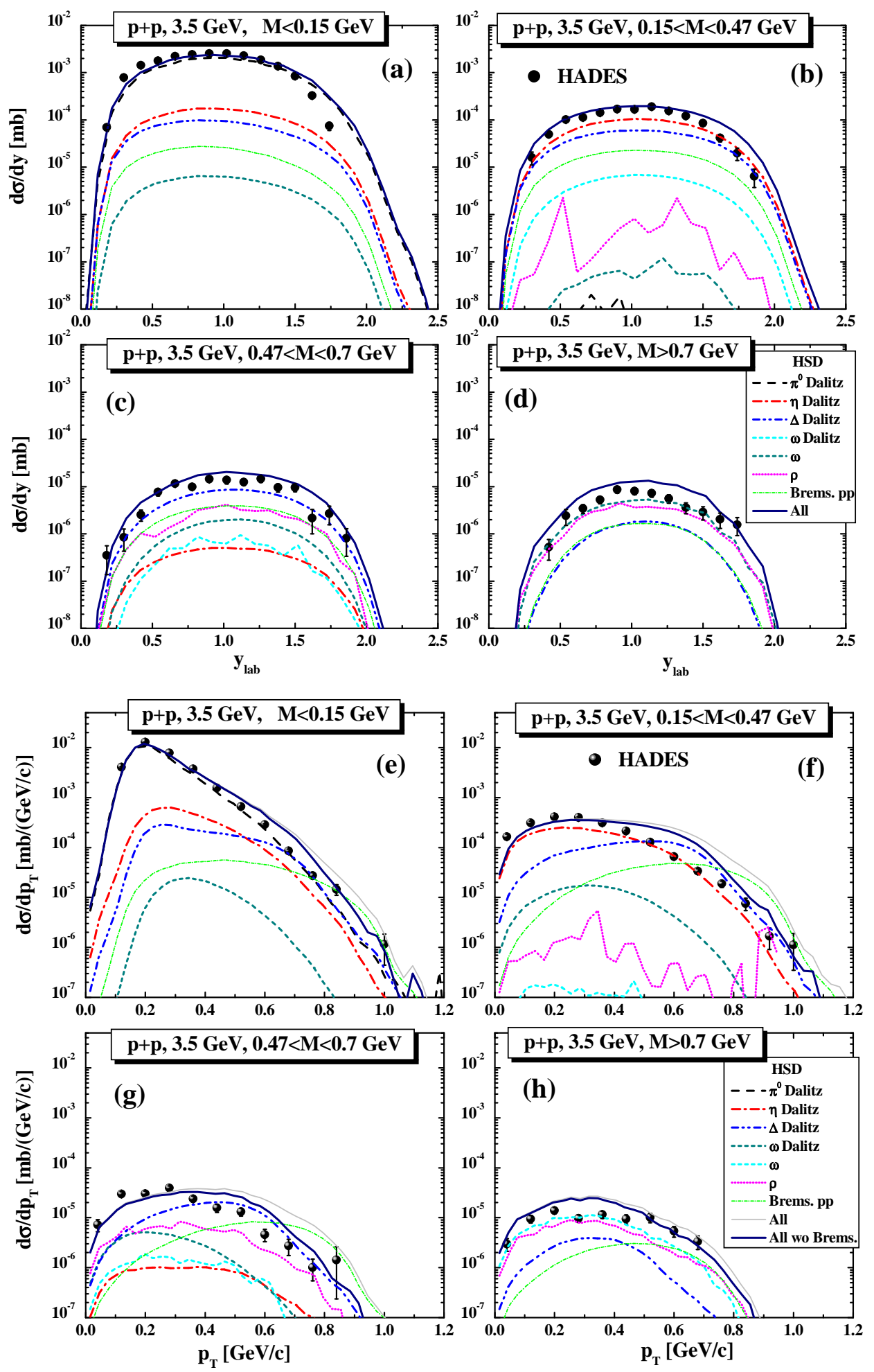

FIG. 12: (Color online) The HSD results for the rapidity distribution (upper 4 plot (a-d)) and the transverse momentum spectra (lower 4 plots $(\mathrm{c}-\mathrm{h})$ ) for $p p$ at $3.5 \mathrm{GeV}$ and for 4 different mass bins: $M \leq 0.15 \mathrm{GeV}, 0.15 \leq M \leq 0.47 \mathrm{GeV}, 0.47 \leq M \leq 0.7$ $\mathrm{GeV}$ and $M \geq 0.7 \mathrm{GeV}$ in comparison to the HADES data [57]. The individual coloured lines display the contributions from the various channels in the HSD calculations (see colour coding in the legend). The tick lines, labelled in the legend as "All wo Brems", show the sum of all channels (labelled as "All") without $p p$ Bremsstrahlung. The theoretical calculations passed through the corresponding HADES acceptance filters and mass/momentum resolutions. 

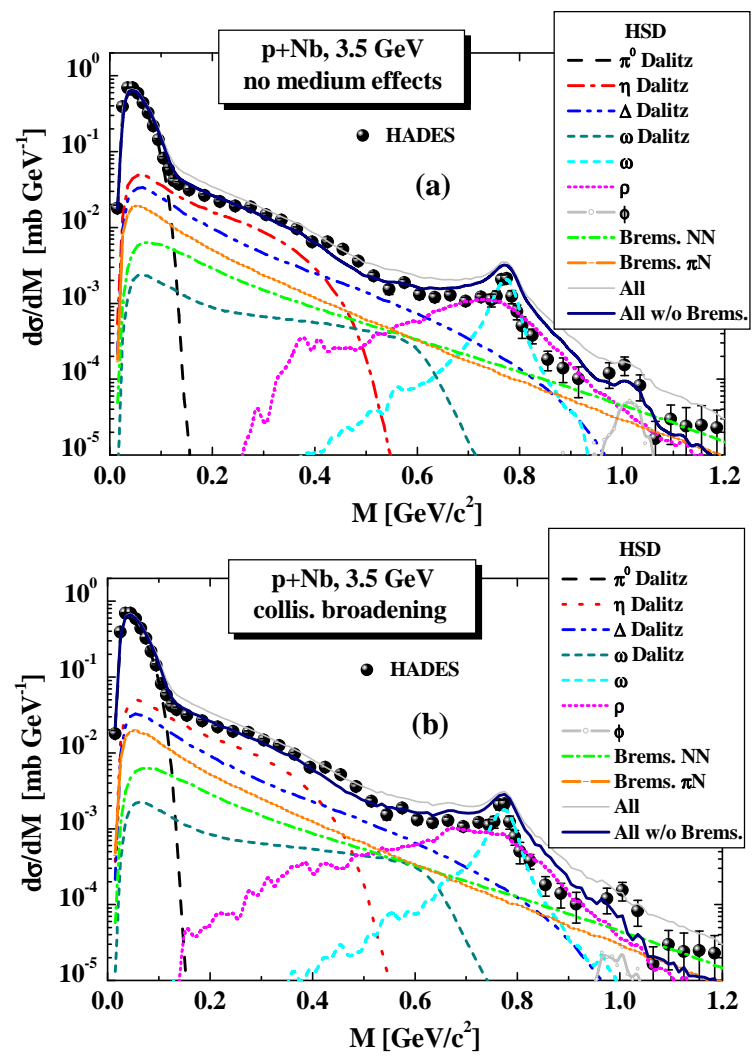

FIG. 13: (Color online) The differential cross section $d \sigma / d M$ from HSD calculations for $e^{+} e^{-}$production in the $p+N b$ reaction at a bombarding energy of $3.5 \mathrm{AGeV}$ in comparison to the HADES data [79]. The upper part (a) shows the case of 'free' vector-meson spectral functions while the lower part (b) gives the result for the 'collisional broadening' scenario. The individual coloured lines display the contributions from the various channels in the HSD calculations (see colour coding in the legend). The tick lines, labelled in legend as "All wo Brems", show the sum of all channels (labelled as "All") without $p p$ bremsstrahlung. The theoretical calculations passed through the corresponding HADES acceptance filters and mass/momentum resolutions. 


\section{DILEPTON PRODUCTION IN HEAVY-ION COLLISIONS}

\section{A. Dileptons from the HSD and IQMD models}

Now we come to the heavy-ion results and start with showing in Fig. 14 the mass differential dilepton spectra normalized to the $\pi^{0}$ multiplicity - of HSD calculations for $\mathrm{C}+\mathrm{C}$ at $1.0 \mathrm{AGeV}$ in comparison to the HADES data 34]. The HADES collaboration has obtained the $\pi^{0}$ multiplicity by the average of the multiplicity of charged pions 86 and we apply the same method for the theoretical calculations. The upper part displays the results for 'free' vector-meson spectral functions while the lower part shows the result for the 'collisional broadening' scenario. We note here, and this holds for all dilepton spectra normalized to the number of $\pi^{0}$ 's, that the normalization is done by the total number of $\pi^{0}$ 's in $4 \pi$, i.e. without applying an experimental acceptance. This allows for a direct comparison with the published HADES results.

The $\Delta$ Dalitz decay and bremsstrahlung contributions are the dominant channels and contribute with about the same weight to the invariant mass spectra. For invariant masses $M>0.3 \mathrm{GeV}$ also the subthreshold $\eta$ channel contributes in an important way. The different descriptions of the $\rho$ meson become important only at large invariant masses where no experimental data are available. The figure shows as well the contribution from direct $\rho$ decays when including the $N^{*}(1520)$ resonance which may enhance the $\rho$ meson production at sub-threshold energies as discussed in Section II.B. As seen in the figure, there is indeed a small contribution but not larger than the experimental error bars. At higher energies other channels dominate. Therefore the $N^{*}(1520)$ resonance is not an important source for dilepton production in heavy-ion reactions. Also the 'in-medium' effects due to the collisional broadening of the spectral functions for $\rho$ and $\omega$ mesons is not visible in the final spectra due to the strong contributions from other dilepton sources at low invariant masses where this effect is most pronounced and partly due to the limited experimental mass resolution at high invariant masses which smears out the spectra.

Fig. [15] shows the results of IQMD calculations, including acceptance in the same way as the HSD calculations. It is remarkable that the two quite sophisticated transport theories predict results which are that similar. Even the channel decomposition is very similar what is all but trivial because the invariant mass spectra depend on many details of the reaction. They include the $\Delta$ dynamics in a nucleus, which we will discuss in section $\mathrm{V}$ in more detail, the number of collisions and hence of the spatial distributions of the nucleons in the colliding nuclei, the Fermi momentum and the Pauli blocking of reactions if final state nucleons would be placed in already occupied phase space regions.

Fig. 16] shows the mass differential dilepton spectra - normalized to the $\pi^{0}$ multiplicity - from HSD calculations for $\mathrm{C}+\mathrm{C}$ - at $2 \mathrm{AGeV}$ in comparison to the HADES data [37]. The theoretical calculations passed through the corresponding HADES acceptance filters and mass/momentum resolutions which leads to a smearing of the spectra at high invariant mass and particularly in the $\omega$ peak region. The upper part shows again the case of 'free' vector-meson spectral functions while the lower part presents the result for the 'collisional broadening' scenario. Also here the difference between the in-medium scenarios is of minor importance, partly due to the limited mass resolution which smears out the spectra. Nevertheless, one can conclude that the 'free' calculations predict an enhancement in the region of the $\rho$ mass which is not seen in the experimental data, which are more in favor to the collisional broadening scenario.

Fig. 17 compares the same data with the results from IQMD calculations for $\mathrm{C}+\mathrm{C}-\mathrm{at} 2 A \mathrm{GeV}$ which have been acceptance corrected in the same way as the HSD data. Again we see a very good agreement between the two theoretical approaches. Only the different parametrizations of the $\omega$ cross section yield deviations at invariant masses around $0.77 \mathrm{GeV}$.

Fig. 18 displays the mass differential dilepton spectra - normalized to the $\pi^{0}$ multiplicity - from HSD calculations for $\mathrm{Ar}+\mathrm{KCl}$ at $1.76 \mathrm{AGeV}$ in comparison to the HADES data 39]. The upper part shows again the case of 'free' vector-meson spectral functions while the lower part gives the result for the 'collisional broadening' scenario. Also in this data set the enhancement around the $\rho$ mass is clearly visible. For this heavier system the 'collisional broadening' scenario shows a slightly better agreement with experiment than the 'free' result and we expect that for larger systems the difference between the two approaches increases.

Fig. 19. which presents the IQMD results for this reaction, shows that the agreement between both theories continues also for heavier systems. Again up to invariant masses of $0.7 \mathrm{GeV}$ both invariant mass spectra are almost identical and agree with data. Also the channel decomposition is rather similar. Here one can see again the overestimation of the dilepton yield by IQMD at $\rho$ /omega peak which is related to the enhance $\omega$ production cross section in elementary pn collisions relative to $p p$ collisions due to the isospin model used in IQMD (cf. Section II.C).

The transverse momentum spectra - normalized to the $\pi^{0}$ multiplicity - for $\mathrm{Ar}+\mathrm{KCl}$ at $1.75 A \mathrm{GeV}$ have been measured by the HADES collaboration for 5 different mass bins 39] : bin 1: $M \leq 0.15 \mathrm{GeV}$, bin 2 : $0.13 \leq M \leq$ $0.3 \mathrm{GeV}$, bin $3: 0.3 \leq M \leq 0.45 \mathrm{GeV}$ bin $4: 0.45 \leq M \leq 0.65 \mathrm{GeV}$ and bin $5: M \geq 0.65 \mathrm{GeV}$. Fig. 20 presents the HADES data in comparison with HSD calculations; on the top without medium effect, on the bottom for the dropping mass scenario. We see also here a good agreement between theory and experiment. Thus one can conclude 

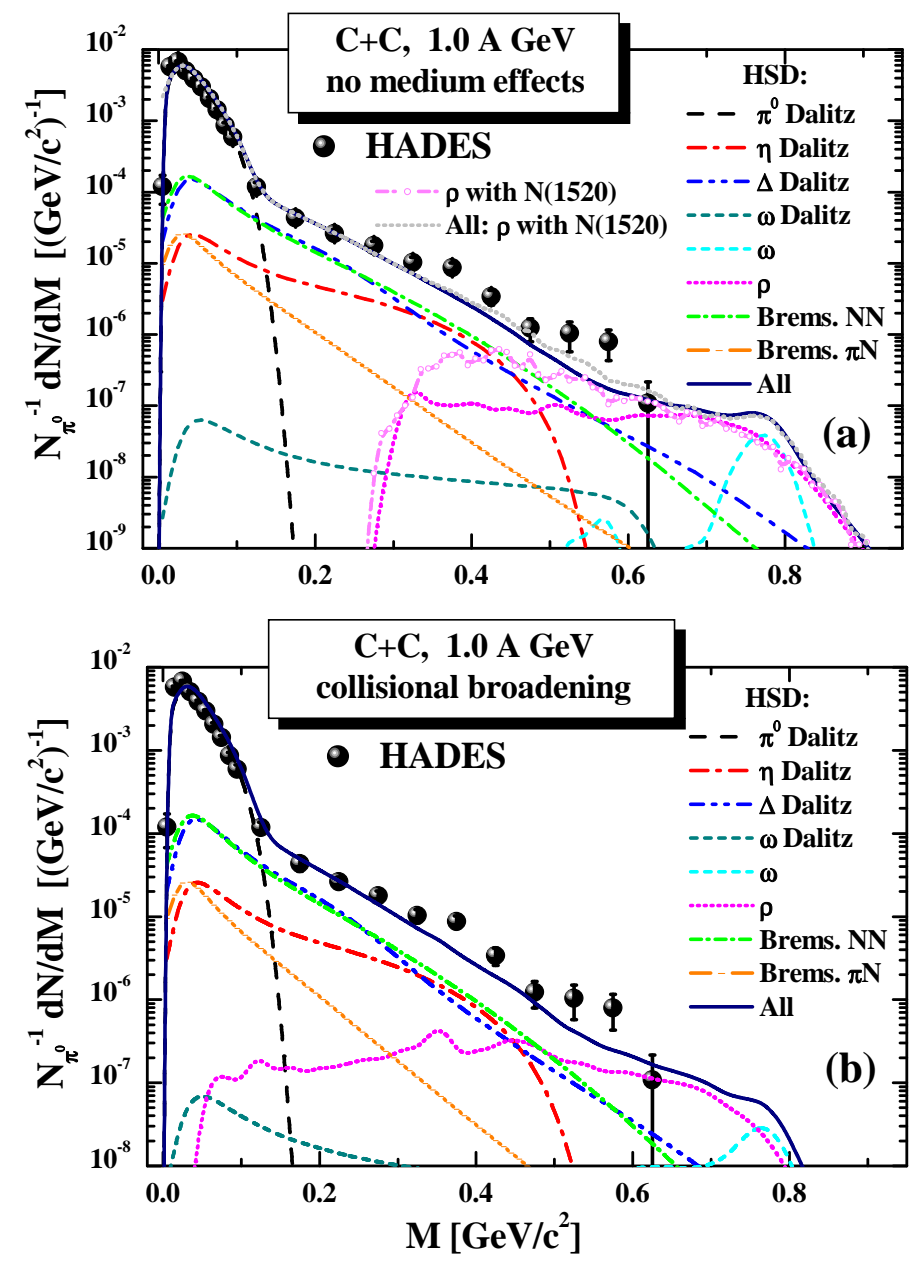

FIG. 14: (Color online) The results of the HSD transport calculation for the mass differential dilepton spectra - normalized to the $\pi^{0}$ multiplicity - for $\mathrm{C}+\mathrm{C}$ at $1.0 \mathrm{AGeV}$ in comparison to the HADES data 34]. The upper part (a) shows the case of 'free' vector-meson spectral functions while the lower part (b) gives the result for the 'collisional broadening' scenario. In both scenarios the HADES acceptance filter and mass/momentum resolution have been incorporated. The different color lines display individual channels in the transport calculation (see legend).

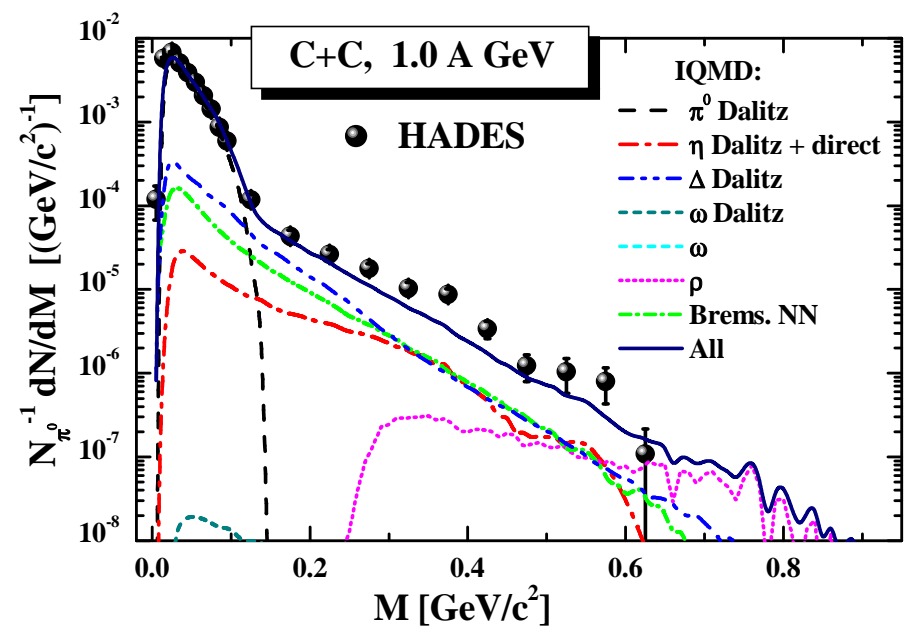

FIG. 15: (Color online) The mass differential dilepton spectra - normalized to the $\pi^{0}$ multiplicity - from IQMD calculations for $\mathrm{C}+\mathrm{C}$ - at $1 \mathrm{AGeV}$ in comparison to the HADES data 34]. The different colour lines display individual channels in the transport calculation (see legend). The theoretical calculations passed through the corresponding HADES acceptance filter and mass/momentum resolutions. 

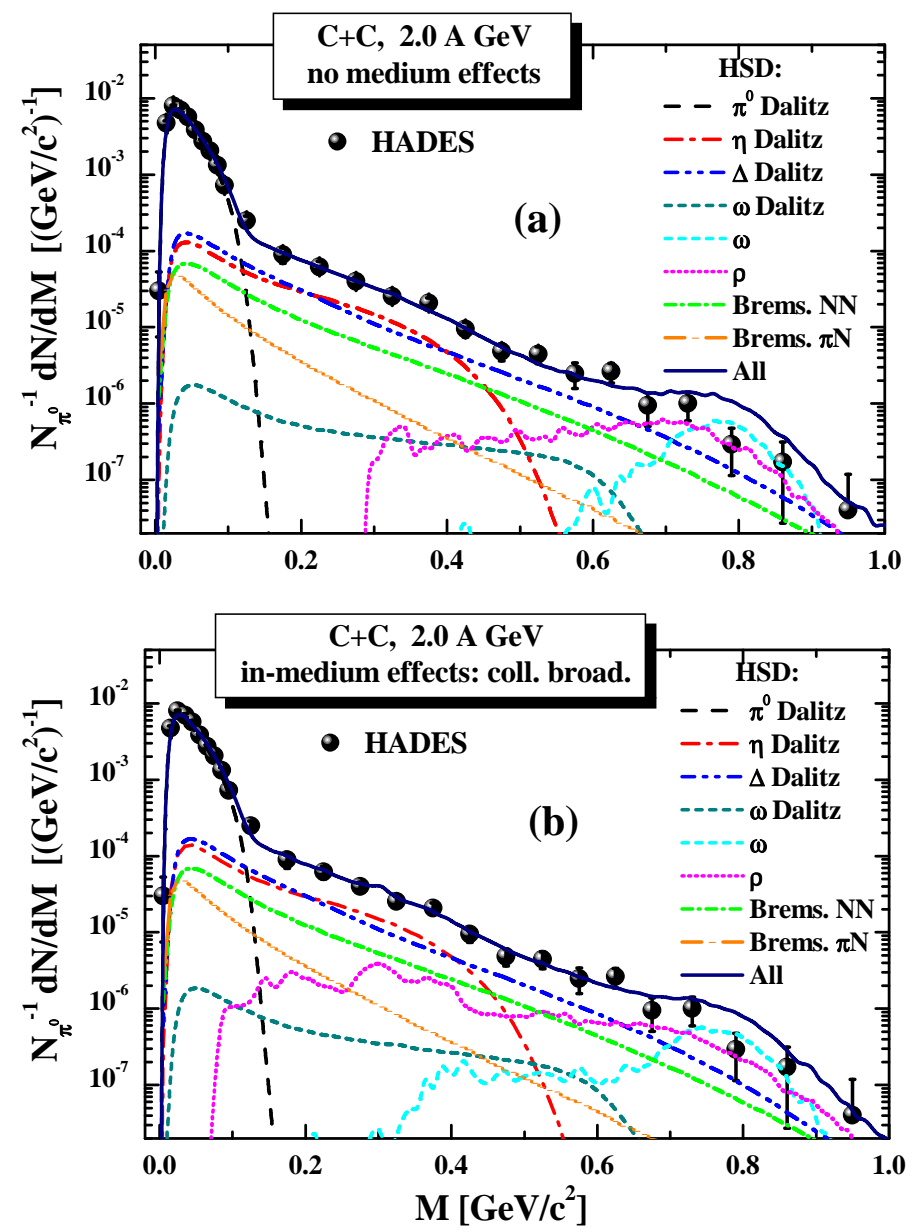

FIG. 16: (Color online) The mass differential dilepton spectra - normalized to the $\pi^{0}$ multiplicity - from HSD calculations for $\mathrm{C}+\mathrm{C}$ at $2 \mathrm{AGeV}$ in comparison to the HADES data [37]. The upper part (a) shows the case of 'free' vector-meson spectral functions while the lower part (b) gives the result for the 'collisional broadening' scenario. The different colour lines display individual channels in the transport calculation (see legend). The theoretical calculations passed through the corresponding HADES acceptance filter and mass/momentum resolutions.

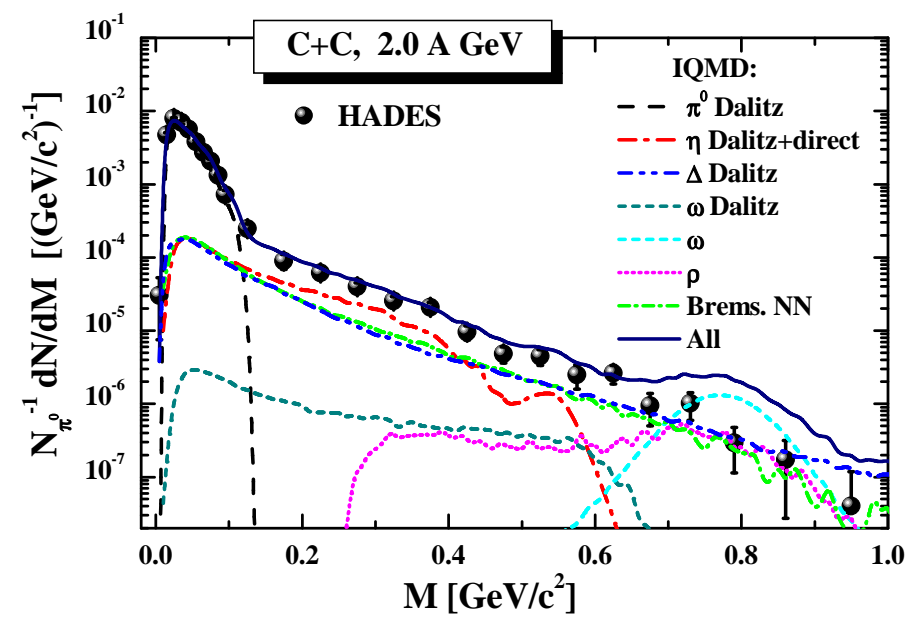

FIG. 17: (Color online) The mass differential dilepton spectra - normalized to the $\pi^{0}$ multiplicity - from IQMD for C+C at 2 $A \mathrm{GeV}$ in comparison to the HADES data 37]. The different colour lines display individual channels in the transport calculation (see legend). The theoretical calculations passed through the corresponding HADES acceptance filter and mass/momentum resolutions. 

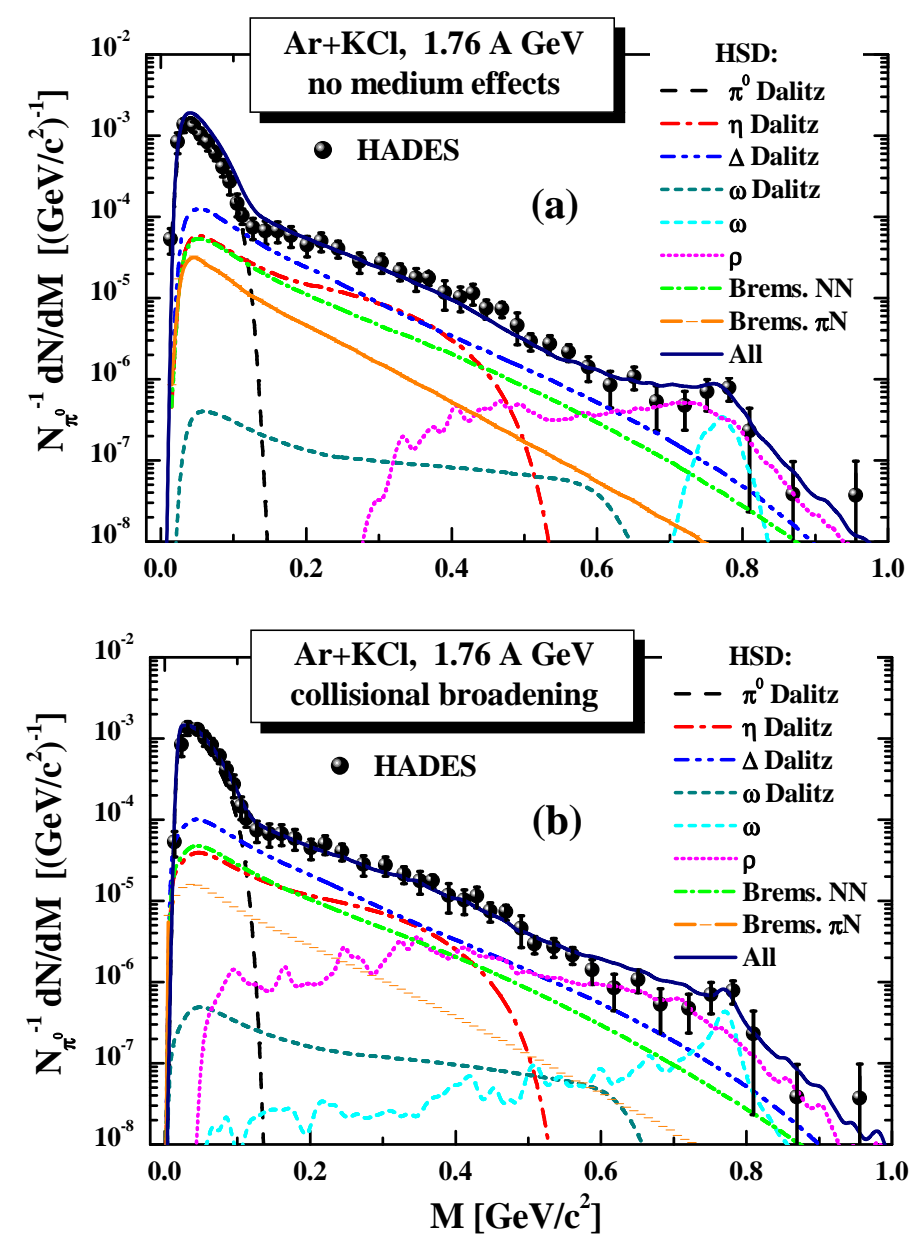

FIG. 18: (Color online) The mass differential dilepton spectra - normalized to the $\pi^{0}$ multiplicity - from $\mathrm{HSD}$ for Ar+KCl at 1.76 AGeV in comparison to the HADES data [39]. The upper part(a) shows the case of 'free' vector-meson spectral functions while the lower part (b) gives the result for the 'collisional broadening' scenario. The individual colored lines display the contributions from the various channels in the HSD calculations (see color coding in the legend). The theoretical calculations passed through the corresponding HADES acceptance filter and mass/momentum resolutions.

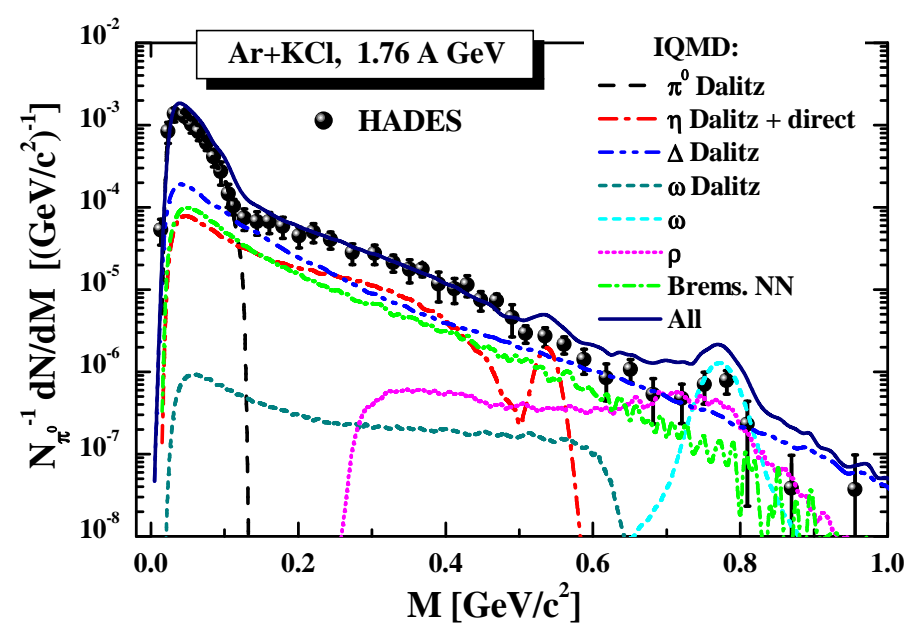

FIG. 19: (Color online) The mass differential dilepton spectra - normalized to the $\pi^{0}$ multiplicity - from IQMD for Ar+KCl at $1.76 \mathrm{AGeV}$ in comparison to the HADES data 39]. The individual colored lines display the contributions from the various channels in the IQMD calculations (see color coding in the legend). The theoretical calculations passed through the corresponding HADES acceptance filter and mass/momentum resolutions. 
that the agreement between theory and experiment ( Fig. 14- Fig. 20) up to $M \approx 0.5 \mathrm{GeV}$ is of such a quality that we can use the theory to study the physical processes involved.
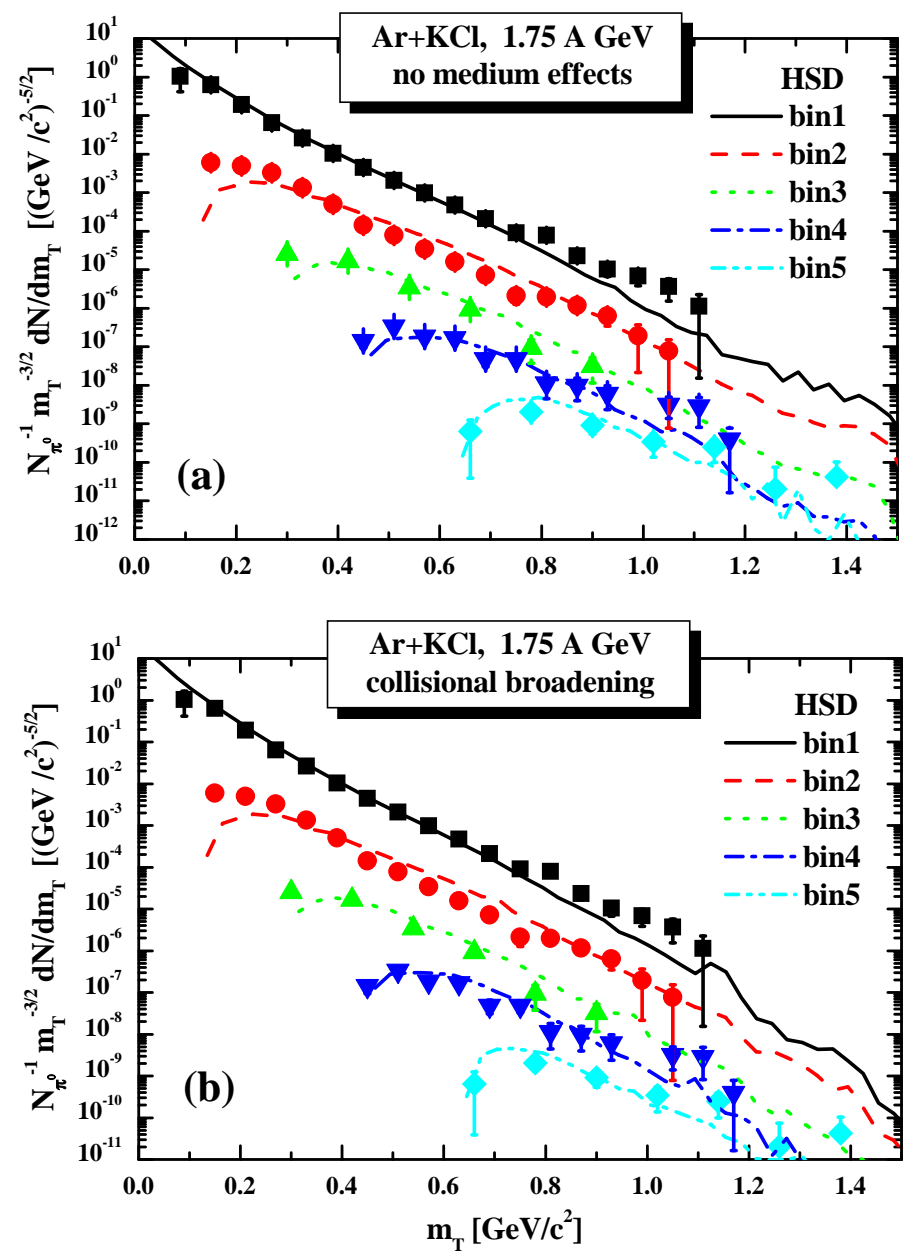

FIG. 20: (Color online) The HSD results for the transverse momentum spectra - normalized to the $\pi^{0}$ multiplicity - for Ar+KCl at $1.75 A \mathrm{GeV}$ for 5 different mass bins: bin $1: M \leq 0.15 \mathrm{GeV}$, bin 2 : $0.13 \leq M \leq 0.3 \mathrm{GeV}$, bin $3: 0.3 \leq M \leq 0.45 \mathrm{GeV}$ bin 4: $0.45 \leq M \leq 0.65 \mathrm{GeV}$ and bin $5: M \geq 0.65 \mathrm{GeV}$ in comparison to the HADES data [39]. The upper part (a) shows the case of 'free' vector-meson spectral functions while the lower part (b) gives the result for the 'collisional broadening' scenario. The individual coloured lines display the contributions from the various channels in the HSD calculations (see colour coding in the legend). The theoretical calculations passed through the corresponding HADES acceptance filter and mass/momentum resolutions.

The HADES collaboration has recently measured also the dilepton invariant mass spectra for the reaction $\mathrm{Au}+\mathrm{Au}$ at $1.25 \mathrm{AGeV}$. The analysis is not completed yet. Fig. 21 presents the HSD predictions for the mass differential dilepton spectra - normalized to the $\pi^{0}$ multiplicity - for this reaction. The upper part shows the case of 'free' vector-meson spectral functions while the lower part gives the result for the 'collisional broadening' scenario.

\section{B. Dileptons from the UrQMD model}

In this subsection we present the results from the UrQMD (v. 2.3) transport model [71, 72]. In this model the dilepton afterburner does not contain bremsstrahlung. It is, however, useful to verify whether it agrees with HSD and IQMD calculations as far as all hadronic dilepton sources are concerned. For the details of the dilepton treatment in UrQMD at SIS energies we refer the reader to Refs. [83, 84].

Fig. 22 shows the mass differential dilepton spectra - normalized to the $\pi^{0}$ multiplicity - from UrQMD calculations for $\mathrm{C}+\mathrm{C}$ - at $2 \mathrm{AGeV}$ in comparison to the HADES data [37] and Fig. 23] - for $\mathrm{Ar}+\mathrm{KCl}$ at $1.76 \mathrm{AGeV}$ in comparison to the HADES data [39]. As one can see from Figs. 222 and 23 the UrQMD v. 2.3 substantially overestimates the 

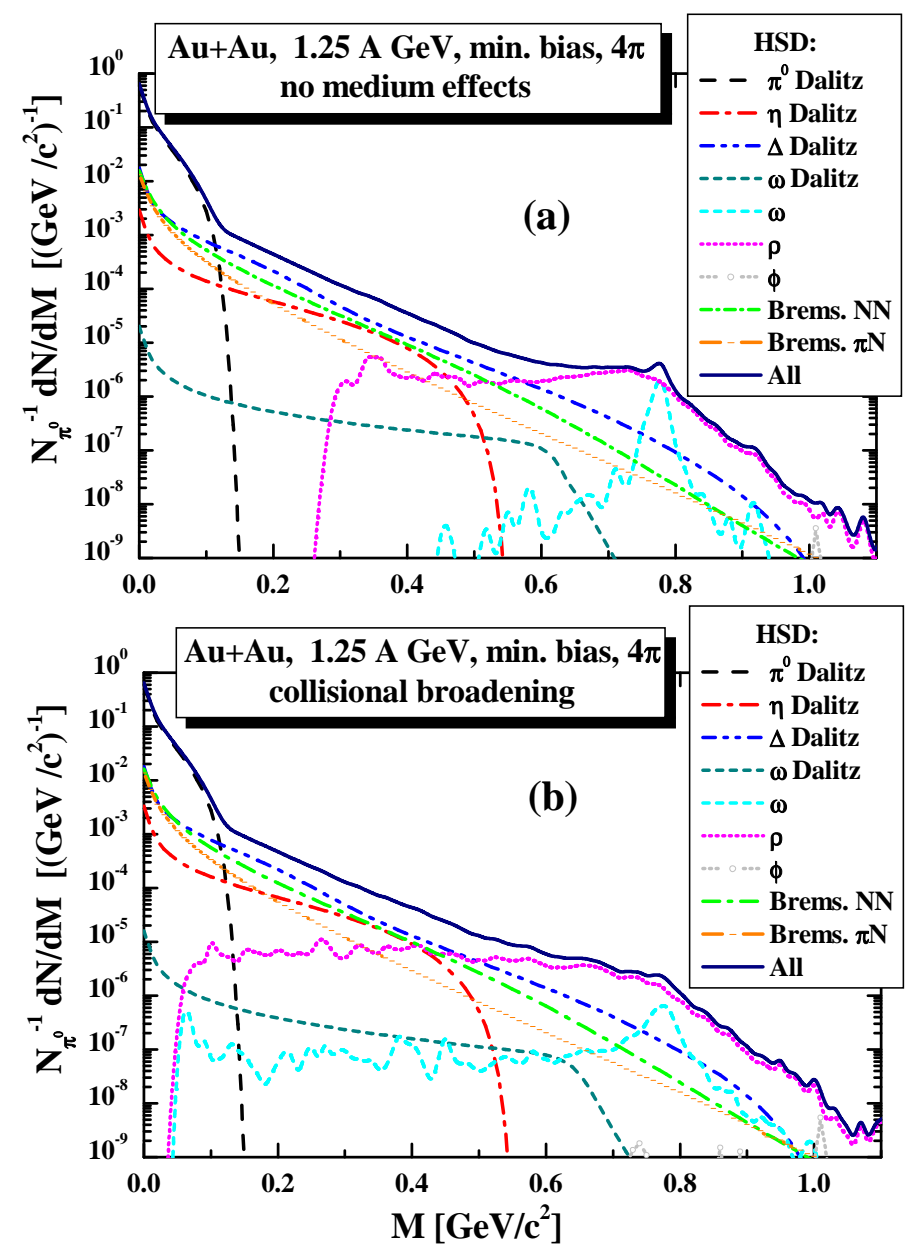

FIG. 21: (Color online) The mass differential dilepton spectra - normalized to the number of $\pi^{0}$ 's - from HSD for minimal bias $\mathrm{Au}+\mathrm{Au}$ collisions at $1.25 \mathrm{AGeV}$. The upper part (a) shows the case of 'free' vector-meson spectral functions while the lower part (b) gives the result for the 'collisional broadening' scenario. The different color lines display individual channels in the transport calculation (see legend).

dilepton yield from the vector mesons. The problem can be traced back to the description of $\rho$ production in elementary $N N$ collisions which proceeds via an excitation and decay of heavy baryonic resonances $N(1520), N(1770), \ldots$. Their coupling to the $\rho$ channel is not well known and may therefore be overestimated. On the other hand the dilepton yield at low invariant masses is underestimated for both systems. This is, first of all, due to the lack of the bremsstrahlung contributions but also due to an underprediction of the $\eta$ yield in UrQMD.

We note that the UrQMD model is presently under improvement and extension, updated results for the dileptons at SIS energies are expected soon [85]. 


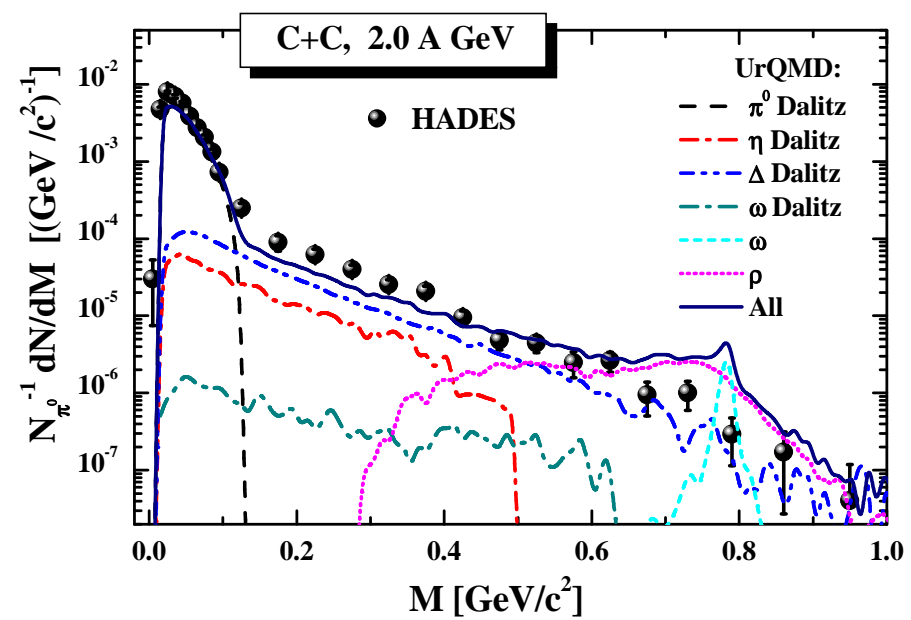

FIG. 22: (Color online) The mass differential dilepton spectra - normalized to the number of $\pi^{0}$ 's - from UrQMD for C+C - at $2 \mathrm{AGeV}$ in comparison to the HADES data [37]. The different colour lines display individual channels in the transport calculation (see legend). The theoretical calculations passed through the corresponding HADES acceptance filter including mass/momentum resolutions.

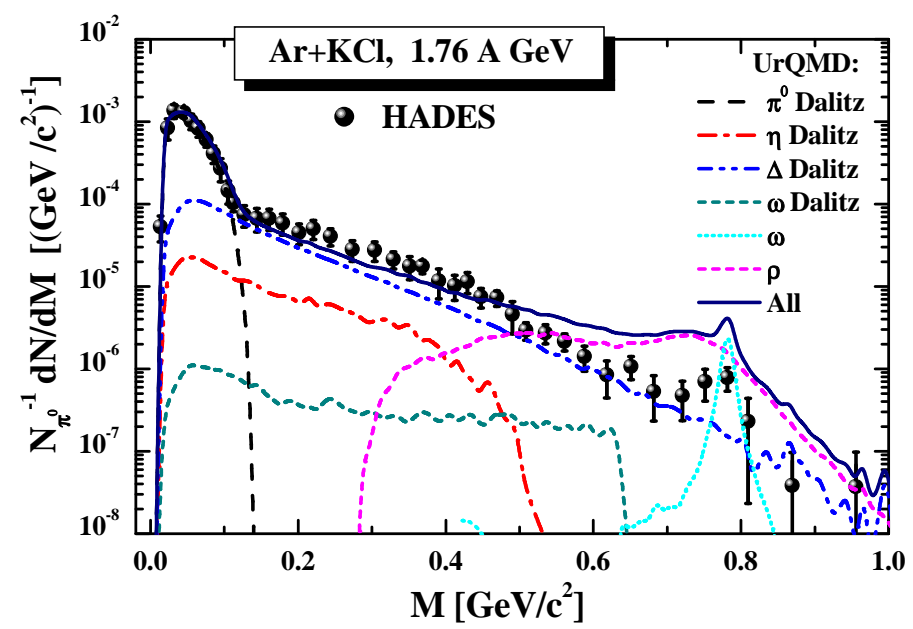

FIG. 23: (Color online) The mass differential dilepton spectra - normalized to the number of $\pi^{0}$ 's - from UrQMD for Ar+KCl at $1.76 \mathrm{AGeV}$ in comparison to the HADES data [39]. The individual colored lines display the contributions from the various channels in the HSD calculations (see color coding in the legend). The theoretical calculations passed through the corresponding HADES acceptance filter including mass/momentum resolutions.

\section{RATIOS OF DILEPTON YIELDS R(AA/NN)}

\section{A. Comparison with experimental data}

The primary interest of measuring dilepton production in heavy-ion collisions is to see whether it is a mere superposition of the production in elementary $(p p+p n(d))$ collisions. Of course in this threshold energy regime the Fermi motion of the nucleons inside a nucleus plays an important role and therefore the question has to be formulated more precisely: Is there an in medium enhancement beyond the Fermi motion? This question we will address in this section.

The HADES collaboration has measured the elementary reactions at different beam energies than the heavy-ion reactions, i.e. $p p$ and quasi-free $p n$ reactions at $1.25 \mathrm{GeV}$ whereas the $\mathrm{C}+\mathrm{C}$ collisions at 1.0 and $2.0 \mathrm{AGeV}$ and $\mathrm{Ar}+\mathrm{KCl}$ at $1.75 \mathrm{AGeV}$. Thus, a comparison of elementary reaction data with those of heavy ions at the same energy was not possible experimentally. Therefore, we also have to calculate the 'reference spectrum' $N N=(p p+p n) / 2$ at $1.25 \mathrm{GeV}$ in order to compare with experimental $A A / N N$ ratios. Then we show the sensitivity of the ratio $A A / N N$ to the energy selection of reference spectra $N N$ which finally might influence the interpretation of in-medium modifications in $A+A$ collisions relative to the $N N$. All calculations presented here have been performed with free vector meson 
spectral functions.

Fig. 24], left, shows the mass differential dilepton spectra - normalized to the multiplicity of $\pi^{0}$ 's and after $\eta$ Dalitz yield subtraction - from HSD calculations for $\mathrm{C}+\mathrm{C}$ at $1.0 \mathrm{AGeV}$ (solid line), for the isospin-averaged reference spectra $N N=(p p+p n) / 2$ at $1.25 \mathrm{GeV}$ (short dashed line) and at $1.0 \mathrm{GeV}$ (dashed line) as well as for $p d$ at $1.25 \mathrm{GeV}$ (dot-dashed line). These calculations are compared to the corresponding HADES data from Refs. 37] - for $\mathrm{C}+\mathrm{C}$ at $1.0 \mathrm{AGeV}$ and the 'reference' spectra taken as an averaged sum of $p p$ and quasi-free $p n(d)($ denoted as $(p p+p n(d)) / 2)$ measured at $1.25 \mathrm{GeV}$. The theoretical calculations passed through the HADES acceptance filter for $\mathrm{C}+\mathrm{C}$ at 1.0 $A \mathrm{GeV}$ (denoted as "acc:CC@1 AGeV") and mass/momentum resolutions which smears out the high mass region. The theoretical reference spectra is taken as the averaged sum of dilepton spectra from $p+p$ and free $p+n$ collisions. As seen from the figure there is no essential difference between our theoretical $p d$ and $N N$ spectra up to $M \approx 0.5 \mathrm{GeV}$ and only for larger invariant masses the enhanced 'open' phase space for $p d$ compared to $N N$ becomes important.

Fig. 24 (r.h.s.) shows the ratio of the dilepton differential spectra for $\mathrm{C}+\mathrm{C}$ at $1.0 \mathrm{AGeV}$ to the isospin-averaged $N N=(p p+p n) / 2$. Both spectra are normalized to the $\pi^{0}$ multiplicity and the $\eta$ Dalitz yield has been subtracted. The solid and short dashed line present the ratio of $\mathrm{C}+\mathrm{C}$ at $1.0 \mathrm{AGeV}$ to $N N$ at $1.25 \mathrm{GeV}$ in the acceptance region and in $4 \pi$, respectively. The dash-dotted and dashed lines are the corresponding ratios of $\mathrm{C}+\mathrm{C}$ at $1.0 \mathrm{AGeV}$ to $\mathrm{NN}$ at $1.0 \mathrm{GeV}$. If we divide the spectra of $\mathrm{C}+\mathrm{C}$ at $1.0 \mathrm{AGeV}$ by the $N N$ spectra at $1.25 \mathrm{GeV}$ the ratio is quite flat, as the experiments show as well. The enhancement in the theory at the upper end of the $\pi^{0}$ peak and hence around $\mathrm{M}=0.15 \mathrm{GeV}$ comes in about equal parts from bremsstrahlung and $\Delta$ Dalitz decay. We observe as well that the acceptance cuts do not change the enhancement. Therefore we can discuss it in the next section using $4 \pi$ yields. In this figure we display as well that the true enhancement, obtained by comparing $\mathrm{C}+\mathrm{C}$ and $N N$ at the same energy, is much larger. For $0.125 \mathrm{GeV}<M<0.3 \mathrm{GeV}$ it is about a factor of two.

We note that the HADES collaboration used $p p$ and quasi-free $p n(d)$ spectra at $1.25 \mathrm{GeV}$ as a reference $N N^{d}=$ $(p p+p n(d)) / 2$ spectrum for the ratios of the dilepton yields from $A A$ to $N N$. In order to avoid the additional uncertainties of dilepton production in $p d$ collision, a system which cannot be modeled reasonably good in semiclassical approaches, we use the reference spectra $N N=(p n+p p) / 2$. As Fig. 24 shows, both methods are equivalent up to invariant masses of $\mathrm{M}=0.4 \mathrm{GeV}$. Above this value the ratio increases very fast because in the elementary reactions the limitation due to phase space is more severe than in heavy-ion collisions, where the Fermi motion can provide larger invariant masses. These HSD results are confirmed by the IQMD calculations shown in Fig. 25 in a form equivalent to Fig. 24.

Now we step to the energy $2.0 \mathrm{AGeV}$. In order to compare the experimental data for $\mathrm{C}+\mathrm{C}$, measured at two different energies 1.0 and $2.0 \mathrm{AGeV}$, the HADES collaboration transformed the $\mathrm{C}+\mathrm{C}$ data measured at $2.0 \mathrm{AGeV}$ to the acceptance of $\mathrm{C}+\mathrm{C}$ at $1.0 \mathrm{AGeV}$ by using - due to lack of statistics - a one-dimensional transformation (see Ref. [37]). We denote this transformation as "1D-acc:CC@1AGeV" in order to distinguish it from the standard three dimensional filtering procedure using the "3D" (defined above as "acc:CC@1AGeV") experimental acceptance matrix (which depends on $M, p_{T}$ and $y$ ), provided by the HADES Collaboration [88] for the filtering of theoretical $4 \pi$ results.

Fig. 26 presents for $\mathrm{C}+\mathrm{C}$ at $2.0 \mathrm{AGeV}$ the same quantities as Fig. 24 for $\mathrm{C}+\mathrm{C}$ at $1.0 \mathrm{AGeV}$. The solid line on the left is the result of the HSD calculations, the short dashed line and the dashed dotted line are the isospinaveraged reference spectra $N N=(p p+p n) / 2$ at $1.25 \mathrm{GeV}$ and $p d$ at $1.25 \mathrm{GeV}$. The dashed line is the reference $N N$ spectrum at $2.0 \mathrm{GeV}$; the corresponding HADES data are taken from Refs. 37]. Note, that the simulated HSD mass distribution for $\mathrm{C}+\mathrm{C}$ at $2.0 \mathrm{AGeV}$ has been transformed to the corresponding acceptance in the same way as done for the experimental data using the "1D-acc:CC@1AGeV" transformation. Fluctuations introduced by this procedure result in part from the limited statistics of the relevant HADES $\mathrm{C}+\mathrm{C}$ data set and in part from the necessary re-binning of the latter.

The right part of Fig. 26] shows the ratio of the dilepton differential spectra - normalized to the $\pi^{0}$ multiplicity and after $\eta$ Dalitz yield subtraction - of $\mathrm{C}+\mathrm{C}$ at $2.0 \mathrm{AGeV}$ to the isospin-averaged reference spectra $N N=(p p+p n) / 2$ taken at $1.25 \mathrm{GeV}$, applying the $\mathrm{C}+\mathrm{C}$ at $2.0 \mathrm{AGeV}$ "1D - acc : CC@1 AGeV" experimental acceptance (solid line), and in $4 \pi$ result with the default "Wolf" differential electromagnetic width for $\Delta$ Dalitz decay (short dashed line) and "Krivoruchenko" width (dash-dot-dotted line) in order to demonstrate the model uncertainties (cf. discussion in Section VI). Also the $\mathrm{HSD}$ results for the ratio of $\mathrm{C}+\mathrm{C}$ at $2.0 \mathrm{AGeV}$ to the reference $N N$ spectra, taken at $2.0 \mathrm{GeV}$, are shown, including the full "3D"- experimental acceptance (dash-dotted line) and in $4 \pi$ (dashed line). These results show that the experimental data measured up to an invariant mass of $M \approx 0.5 \mathrm{GeV}$ are compatible with a ratio of one and hence with no in-medium enhancement. The theoretical results are more complicated. Up to an invariant mass of $M \approx 0.3 \mathrm{GeV}$ theory predicts a enhancement factor of about 1.8 for $4 \pi$. The ratio at the same nominal energy shows this enhancement even up to invariant masses of $M \approx 0.6 \mathrm{GeV}$ before the influence of the Fermi motion sets in.

The IQMD calculations for $\mathrm{C}+\mathrm{C}$ at $2.0 \mathrm{AGeV}$ are presented in Fig. 27 which shows the same quantities as Fig. 26. We see that the both model agree quite well and the form of the ratio is identical in both approaches.

Fig. 28 (l.h.s.) displays the mass differential dilepton spectra for $\mathrm{Ar}+\mathrm{KCl}$ at $1.76 \mathrm{AGeV}$ (solid line) - normalized 

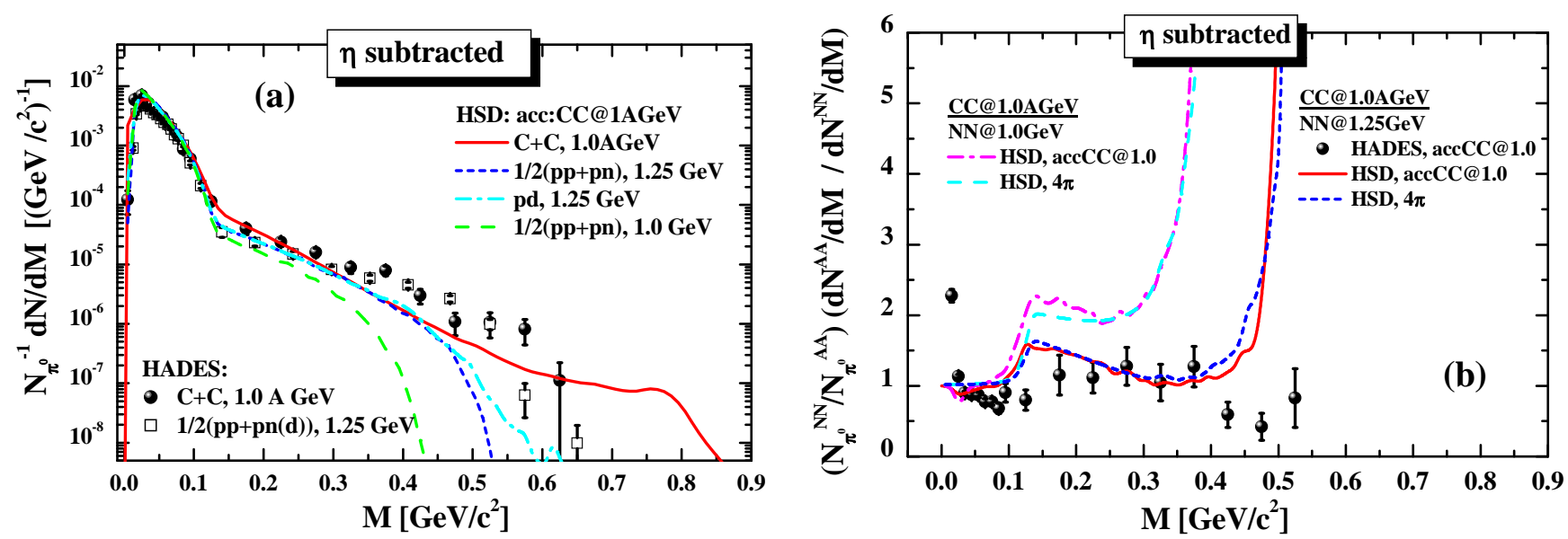

FIG. 24: (Color online) Left (a): The mass differential dilepton spectra - normalized to the $\pi^{0}$ multiplicity and after $\eta$ Dalitz yield subtraction - from HSD calculations for $\mathrm{C}+\mathrm{C}$ at $1.0 \mathrm{AGeV}$ (solid line), for the isospin-averaged reference spectra $N N=(p p+p n) / 2$ at $1.25 \mathrm{GeV}$ (short dashed line) and at $1.0 \mathrm{GeV}$ (dashed line) as well as for $p d$ at $1.25 \mathrm{GeV}$ (dot-dashed line) in comparison to the corresponding HADES data 37] - for $\mathrm{C}+\mathrm{C}$ at $1.0 \mathrm{AGeV}$ and the 'reference' spectra taken as an averaged sum of $p p$ and quasi-free $p n(d)$ (denoted as $(p p+p n(d)) / 2)$ measured at $1.25 \mathrm{GeV}$. The theoretical calculations passed through the HADES acceptance filter for $\mathrm{C}+\mathrm{C}$ at $1.0 \mathrm{AGeV}$ (denoted as "acc:CC@1AGeV") and mass/momentum resolutions. Right (b): Ratio of the dilepton differential spectra - normalized to the $\pi^{0}$ multiplicity and after $\eta$ Dalitz yield subtraction to the isospin-averaged reference spectra $N N=(p p+p n) / 2$ taken at $1.25 \mathrm{GeV}$ employing $\mathrm{C}+\mathrm{C}$ at $1.0 \mathrm{AGeV}$ experimental ("acc:CC@1AGeV") acceptance (solid line) and in $4 \pi$ (short dashed line). Also the HSD results for the ratio of C+C at $1.0 \mathrm{AGeV}$ to the reference $N N$ spectra at $1.0 \mathrm{GeV}$ are shown with experimental ("acc:CC@1AGeV") acceptance corrections (dash-dotted line) and in $4 \pi$ (dashed line).
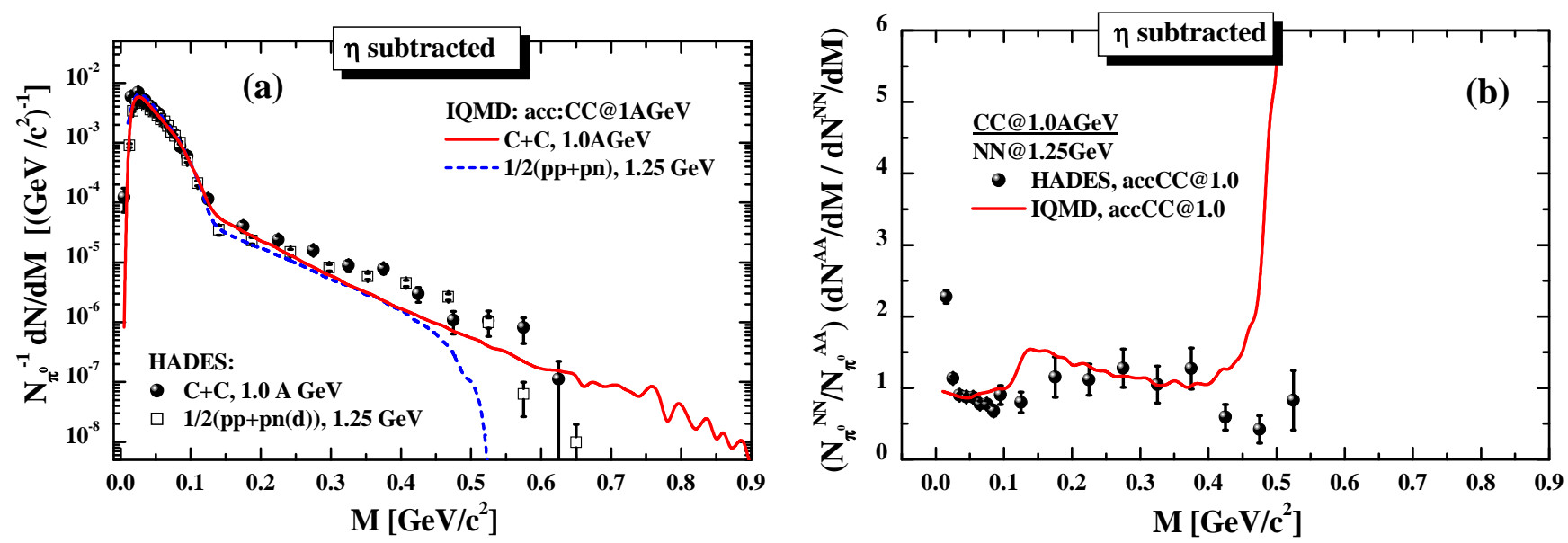

FIG. 25: (Color online) Left (a): The mass differential dilepton spectra - normalized to the $\pi^{0}$ multiplicity and after $\eta$ Dalitz yield subtraction - from IQMD calculations for $\mathrm{C}+\mathrm{C}$ at $1.0 \mathrm{AGeV}$ (solid line), for the isospin-averaged reference spectra $N N=(p p+p n) / 2$ at $1.25 \mathrm{GeV}$ (short dashed line) in comparison to the HADES data 37]. The theoretical calculations passed through the HADES acceptance filter for $\mathrm{C}+\mathrm{C}$ at $1.0 \mathrm{AGeV}$ and mass/momentum resolutions. Right (b): Ratio of the dilepton differential spectra - normalized to the $\pi^{0}$ multiplicity and after $\eta$ Dalitz yield subtraction - of $\mathrm{C}+\mathrm{C}$ at $1.0 A \mathrm{GeV}$ (employing $\mathrm{C}+\mathrm{C}$ at $1.0 \mathrm{AGeV}$ experimental ("acc:CC@1 $\mathrm{AGeV}$ ") acceptance) to the isospin-averaged reference spectra $N N=(p p+p n) / 2$ taken at $1.25 \mathrm{GeV}$.

to the $\pi^{0}$ multiplicity and after $\eta$ Dalitz yield subtraction. We compare HSD calculations for $\mathrm{Ar}+\mathrm{KCl}$ at $1.76 A \mathrm{GeV}$, for the isospin-averaged reference spectra $N N=(p p+p n) / 2$ at $1.25 \mathrm{GeV}$ (short dashed line) and at $1.76 \mathrm{GeV}$ (dashed line) as well as for $p d$ at $1.25 \mathrm{GeV}$ (dot-dashed line) to the corresponding HADES data, taken from Ref. [39]. The theoretical calculations for $\mathrm{Ar}+\mathrm{KCl}$ and for $N N$ passed through the HADES acceptance filter for $\mathrm{Ar}+\mathrm{KCl}$ and mass/momentum resolutions. The right part of Fig. 28 shows the ratio of the dilepton differential spectra - 

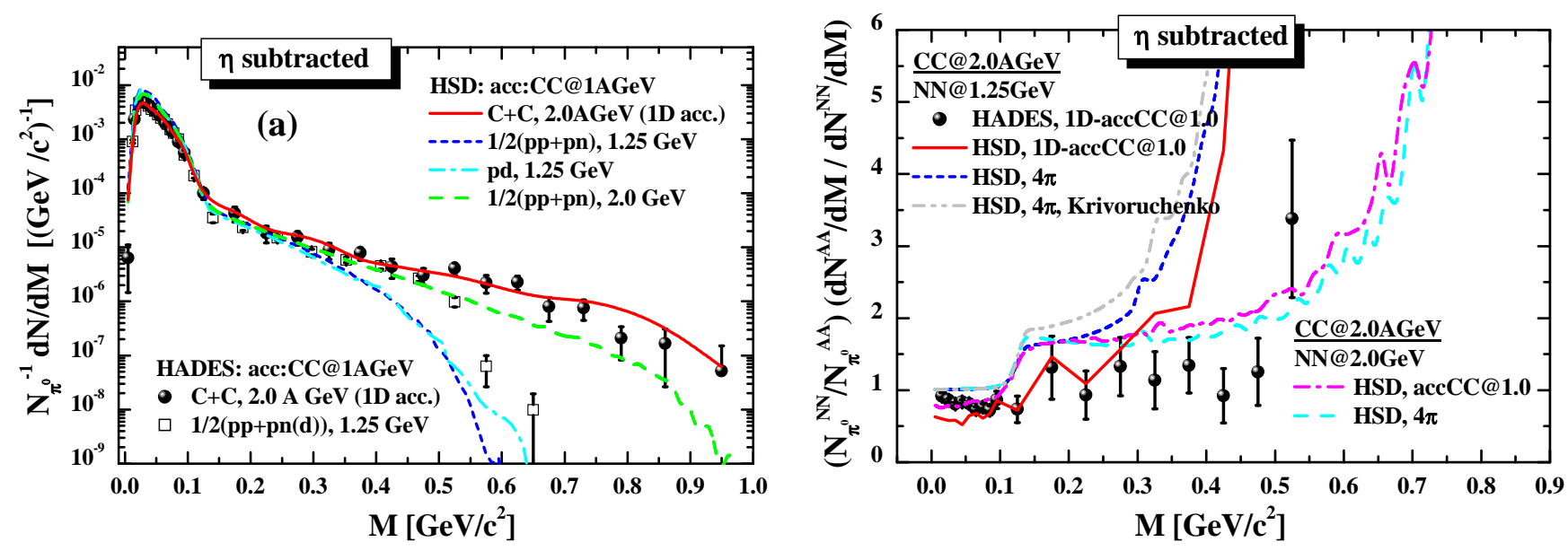

FIG. 26: (Color online) Left (a): The mass differential dilepton spectra - normalized to the $\pi^{0}$ multiplicity and after $\eta$ Dalitz yield subtraction - from HSD calculations for $\mathrm{C}+\mathrm{C}$ at $2.0 \mathrm{AGeV}$ (solid line) and for the isospin-averaged reference spectra $N N=(p p+p n) / 2$ at $1.25 \mathrm{GeV}$ (short dashed line) and at $2.0 \mathrm{GeV}$ (dashed line) as well as for $p d$ at $1.25 \mathrm{GeV}$ (dot-dashed line) in comparison to the HADES data 37] - for $\mathrm{C}+\mathrm{C}$ measured at $2.0 \mathrm{AGeV}$ and $(p p+p n(d)) / 2$ at $1.25 \mathrm{GeV}$ and transformed to the acceptance for $\mathrm{C}+\mathrm{C}$ at $1.0 \mathrm{AGeV}$ (see the discussion in the text). The theoretical calculations passed through the HADES acceptance filter for $\mathrm{C}+\mathrm{C}$ at $1.0 \mathrm{AGeV}$ ("1D-acc:CC@1AGeV") and mass/momentum resolutions. Right (b): Ratio of the dilepton differential spectra of $\mathrm{C}+\mathrm{C}$ at $2.0 \mathrm{AGeV}$ - normalized to the $\pi^{0}$ multiplicity and after $\eta$ Dalitz yield subtraction - to the isospin-averaged reference spectra $N N=(p p+p n) / 2$ at $1.25 \mathrm{GeV}$ with experimental ("1D-acc:CC@1AGeV") acceptance (solid line) and in $4 \pi$ result with the default "Wolf" differential electromagnetic width for $\Delta$ Dalitz decay (short dashed line) and "Krivoruchenko" width (dash-dot-dotted line). Also the HSD results for the ratio of C+C at $2 A G e V$ to the reference $N N$ spectra at $2.0 \mathrm{GeV}$ are shown: with experimental ("acc:CC@1AGeV") acceptance for C+C at 1.0 AGeV (dash-dotted line) and in $4 \pi$ (dashed line).
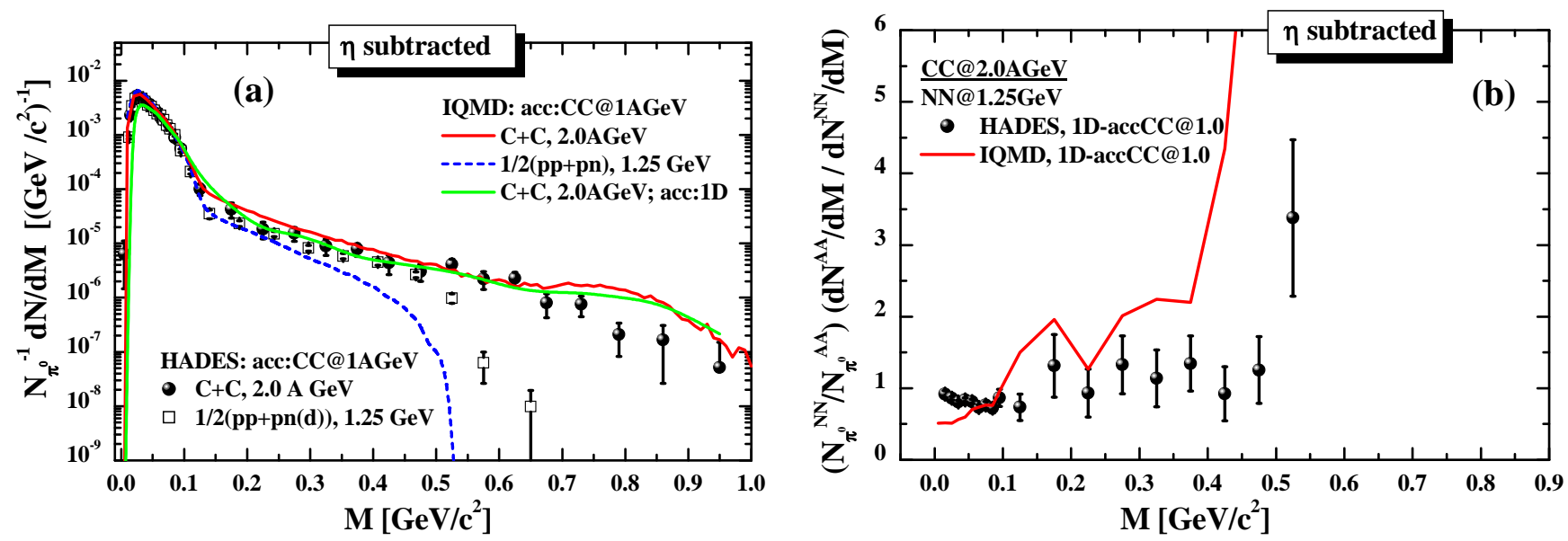

FIG. 27: (Color online) Left (a): The mass differential dilepton spectra - normalized to the $\pi^{0}$ multiplicity and after $\eta$ Dalitz yield subtraction - from IQMD calculations for $\mathrm{C}+\mathrm{C}$ at $2.0 \mathrm{AGeV}$ (solid line) and for the isospin-averaged reference spectra $N N=$ $(p p+p n) / 2$ at $1.25 \mathrm{GeV}$ (short dashed line) in comparison to the HADES data [37]. The theoretical calculations passed through the corresponding HADES acceptance filter for C+C at 1.0 AGeV ("1D-acc:CC@1AGeV") and mass/momentum resolutions (see the discussion in the text). Right (b): Ratio of the dilepton differential spectra for $\mathrm{C}+\mathrm{C}$ at $2.0 A \mathrm{GeV}-$ normalized to the $\pi^{0}$ multiplicity and after $\eta$ Dalitz yield subtraction - to the isospin-averaged reference spectra $N N=(p p+p n) / 2$ taken at 1.25 $\mathrm{GeV}$ with experimental ("1D-acc:CC@1AGeV") acceptance for C+C at $1.0 \mathrm{AGeV}$ (solid line).

normalized to the $\pi^{0}$ multiplicity and after $\eta$ Dalitz yield subtraction - to the isospin-averaged reference spectra $N N=(p p+p n) / 2$ taken at $1.25 \mathrm{GeV}$ and employing the $\mathrm{Ar}+\mathrm{KCl}$ experimental acceptance (solid line) and in $4 \pi$ (short dashed line). We display as well the $\mathrm{HSD}$ results for the ratio of $\mathrm{Ar}+\mathrm{KCl}$ at $1.76 \mathrm{AGeV}$ to the reference $N N$ 

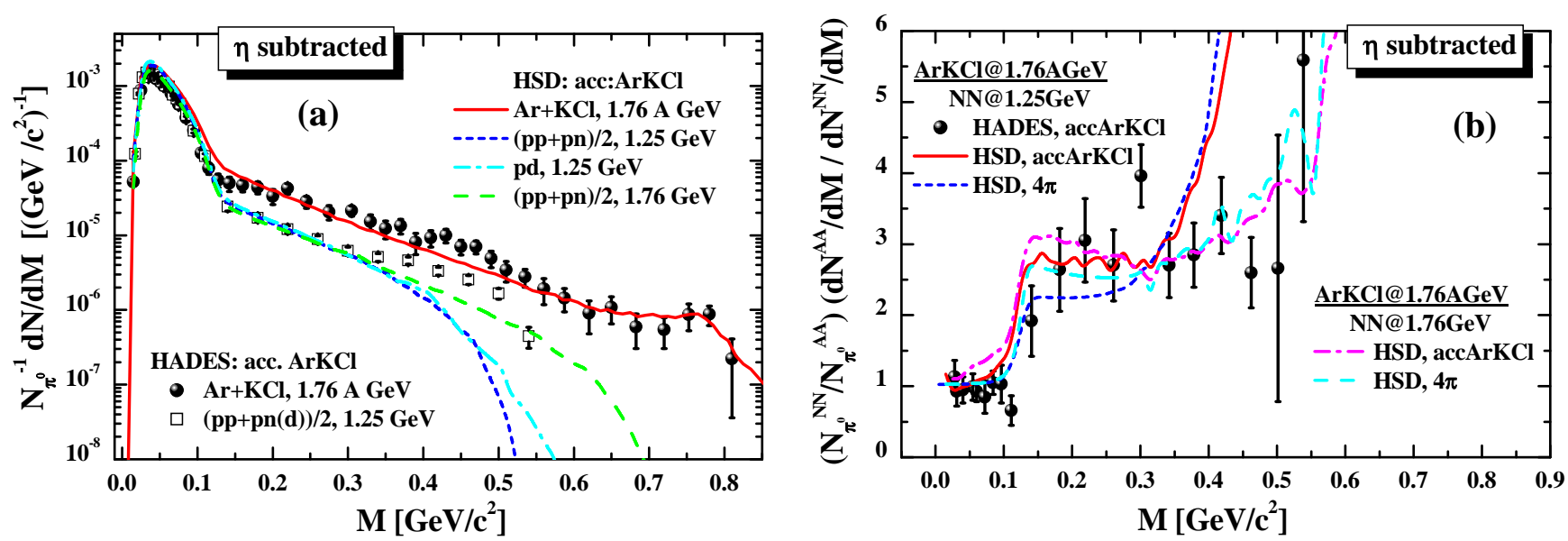

FIG. 28: (Color online) Left (a): The mass differential dilepton spectra - normalized to the $\pi^{0}$ multiplicity and after $\eta$ Dalitz yield subtraction - from HSD calculations for $\mathrm{Ar}+\mathrm{KCl}$ at $1.76 \mathrm{AGeV}$ (solid line) and for the isospin-averaged reference spectra $N N=(p p+p n) / 2$ at $1.25 \mathrm{GeV}$ (short dashed line) and at $1.76 \mathrm{GeV}$ (dashed line) as well as for $p d$ at $1.25 \mathrm{GeV}$ (dot-dashed line) in comparison to the corresponding HADES data [39]. The theoretical calculations for $\mathrm{Ar}+\mathrm{KCl}$ and for $\mathrm{NN}$ passed through the HADES acceptance filter for $\mathrm{Ar}+\mathrm{KCl}$ and mass/momentum resolutions. Right (b): Ratio of the dilepton differential spectra - normalized to the $\pi^{0}$ multiplicity and after $\eta$ Dalitz yield subtraction - to the isospin-averaged reference spectra $N N=(p p+p n) / 2$ taken at $1.25 \mathrm{GeV}$, involving $\mathrm{Ar}+\mathrm{KCl}$ experimental acceptance (solid line) and for $4 \pi$ (short dashed line). Also the HSD results for the ratio to the reference $N N$ spectra taken at $1.76 \mathrm{GeV}$ are shown, with the Ar+KCl experimental acceptance (dash-dotted line) and in $4 \pi$ (dashed line).
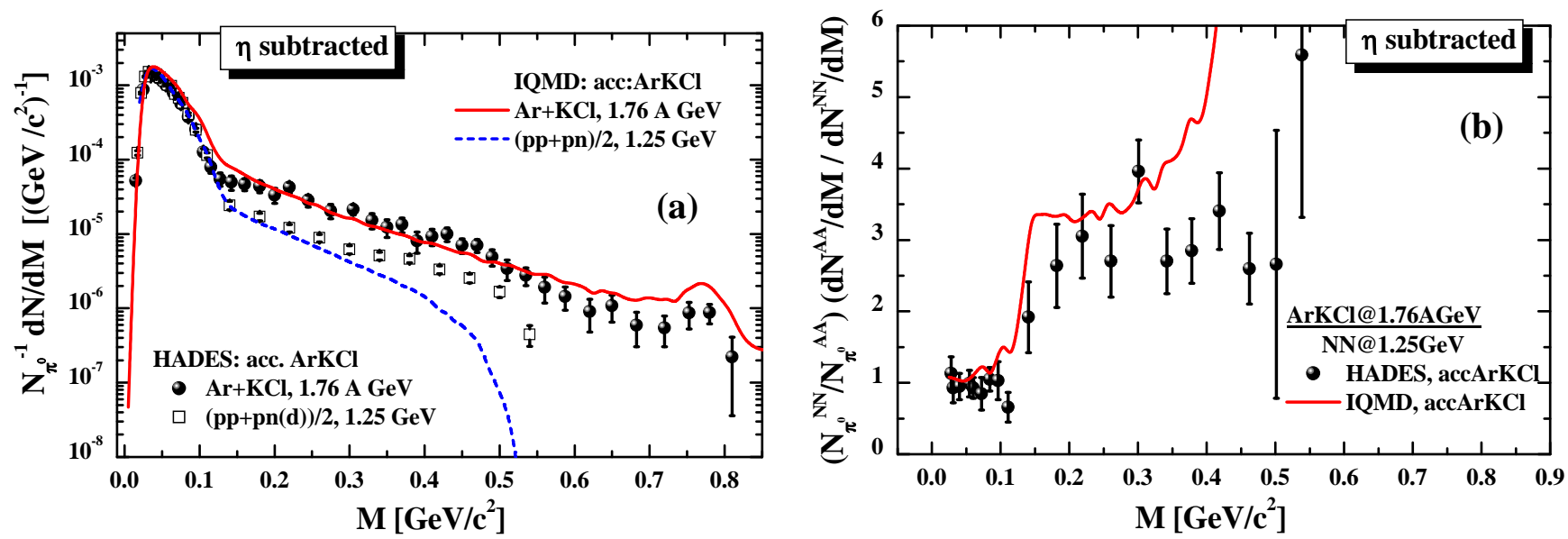

FIG. 29: (Color online) Left (a): The mass differential dilepton spectra - normalized to the $\pi^{0}$ multiplicity and after $\eta$ Dalitz yield subtraction - from IQMD calculations for of $\mathrm{Ar}+\mathrm{KCl}$ at $1.76 \mathrm{AGeV}$ (solid line) and for the isospin-averaged reference spectra $N N=(p p+p n) / 2$ at $1.25 \mathrm{GeV}$ (short dashed line) in comparison to the corresponding HADES data 39]. The theoretical calculations for $\mathrm{Ar}+\mathrm{KCl}$ and for $N N$ passed through the HADES acceptance filter for $\mathrm{Ar}+\mathrm{KCl}$ and mass/momentum resolutions. Right (b): Ratio of the dilepton differential spectra - normalized to the $\pi^{0}$ multiplicity and after $\eta$ Dalitz yield subtraction - to the isospin-averaged reference spectra $N N=(p p+p n) / 2$, taken at $1.25 \mathrm{GeV}$, employing the $\mathrm{Ar}+\mathrm{KCl}$ experimental acceptance (solid line).

spectrum at the same energy, including the experimental $\mathrm{Ar}+\mathrm{KCl}$ acceptance (dash-dotted line) and in $4 \pi$ (dashed line). These results show clearly that for invariant masses of $0.1 \mathrm{GeV}<M<0.35 \mathrm{GeV}$ the data as well as theory are not a mere superposition of the elementary spectra. The comparison also excludes that this enhancement, observed in heavy-ion collisions, is due to acceptance since the results with acceptance and in $4 \pi$ are very similar. At larger invariant masses theory and data do not agree because of the bump at the invariant masses around $M \approx 0.5 \mathrm{GeV}$, seen in the experimental $p d$ reactions, is not reproduced by theory. Taking the reference spectra at the same nominal 
energy theory predicts that this enhancement is constant up to energies of $M \approx 0.5 \mathrm{GeV}$. Then the Fermi motion becomes important and yields a strong increase of the ratio.

Consequently, the experimental ratios of the invariant mass spectra measured in heavy-ion collisions to the isospinaveraged reference spectra $N N=(p p+p n) / 2$ taken at $1.25 \mathrm{GeV}$ reveals an in-medium enhancement in $\mathrm{Ar}+\mathrm{KCl}$ collisions at $1.75 A \mathrm{GeV}$ whereas in $C+C$ collisions at $2 A \mathrm{GeV}$ this ratio is compatible with one and therefore no in-medium enhancement is seen. The transport models show an enhancement in all heavy-ion reactions when the reference spectrum is taken at the same energy. It shows as well that acceptance cuts do not modify this enhancement. The origin of this enhancement will be discussed in the next subsection.

In Fig. 29 we display the same quantities as in Fig. 28 but for IQMD calculations. The enhancement of the experimental ratio is confirmed by IQMD calculations, which are in quantitative agreement with the HSD results.

\section{B. Energy and system size dependence of the dilepton yield}

In this section we present the energy and system size dependence of the dilepton yield in $4 \pi$ as predicted by the HSD calculations in order to study the question of a possible in medium enhancement and to identify eventually its physical origin.
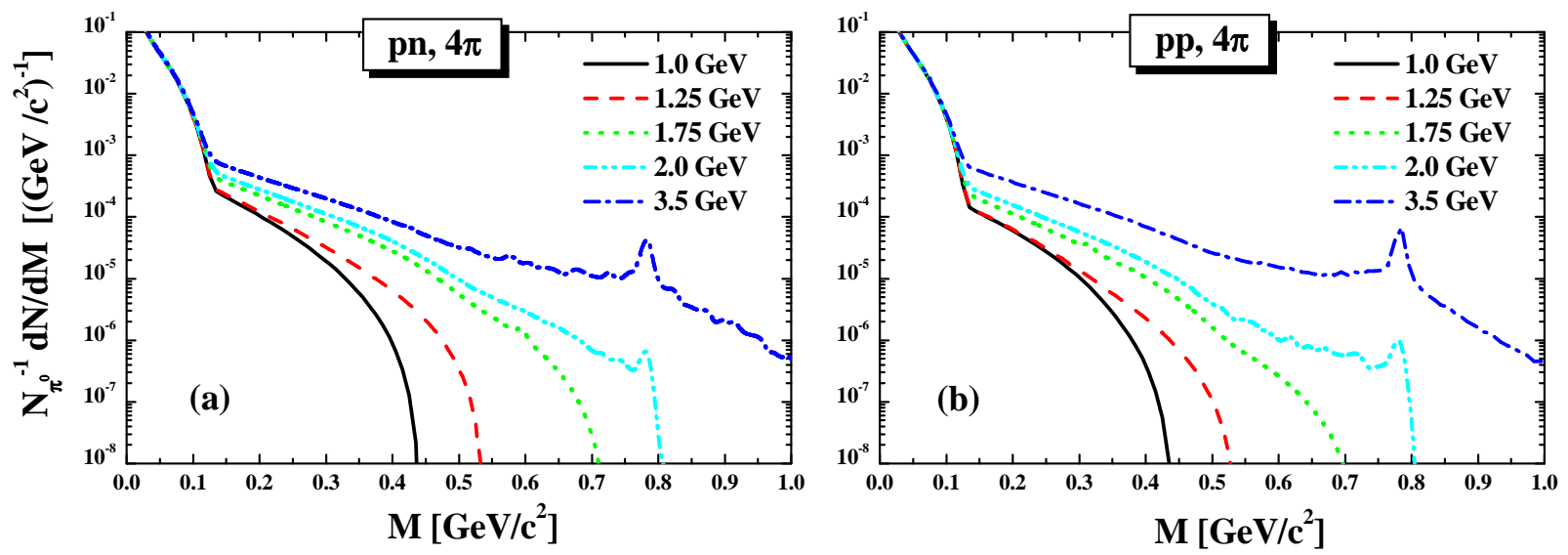

FIG. 30: (Color online) The $4 \pi$ mass differential dilepton spectra - normalized to the $\pi^{0}$ multiplicity - obtained in HSD calculations for $p n$ (left (a)) and $p p$ (right (b)) collisions at 1.0, 1.25, 1.75, 2.0 and $3.5 \mathrm{GeV}$.

Fig. 30 shows the HSD calculations for the mass differential dilepton spectra - normalized to the $\pi^{0}$ multiplicity for $p n$ (left) and $p p$ (right) collisions at 1.0, 1.25, 1.75, 2.0 and $3.5 \mathrm{GeV}$ in $4 \pi$ acceptance. Whereas the normalization renders the low invariant mass part to one, independent of the beam energy, the spectra at high invariant masses show a strong beam energy dependence, as expected. Bremsstrahlung is not coupled to the number of pions (or the number of participants which is often assumed to be proportional to the number of $\pi$ 's) but to the number of collisions. Also the production of heavier mesons increases at these energies close to the meson thresholds, either because it becomes easier to produce them directly or because the baryonic resonances which decay into these resonances are more frequently populated. Last but not least, the phase space limitation of the invariant mass changes with energy which makes ratios between invariant mass spectra at different energies complicated. Due to the isospin dependence of different processes the $p p$ and $p n$ invariant mass spectra differ in detail but are generally determined by phase space. We can conclude from Fig. 30] that the comparison of dilepton data of heavy-ions and of elementary reactions suffer substantially if both are measured at different energies. This renders quantitative conclusions difficult.

Fig. 31 displays the results of HSD calculations for the $4 \pi$ mass differential dilepton spectra - normalized to the $\pi^{0}$ multiplicity - for the minimal bias symmetric heavy-ion collisions as compared to the isospin-averaged reference spectra $N N=(p n+p p) / 2$. We display calculations for $\mathrm{C}+\mathrm{C}, \mathrm{Ar}+\mathrm{KCl}, \mathrm{Au}+\mathrm{Au}$ at 1.0, 1.25, 1.75, 2.0 AGeV . The upper plot corresponds to the total dilepton $A+A$ spectra whereas the lower plot shows the dilepton spectra after $\eta$ Dalitz yield subtraction. The thick lines on the lower plot stand for the $A+A$ dilepton yields whereas the thin lines show the $N N$ spectra at the same energies. We see clearly that the dilepton spectra do not scale with the $\pi^{0}$ multiplicity for invariant masses $M>0.11 \mathrm{GeV}$. There is a strong energy and system size dependence of this invariant mass region due to the complicated dynamics of baryon resonances and mesons. Generally the invariant mass spectra in $A+A$ collisions are smoother due to the Fermi motion. 

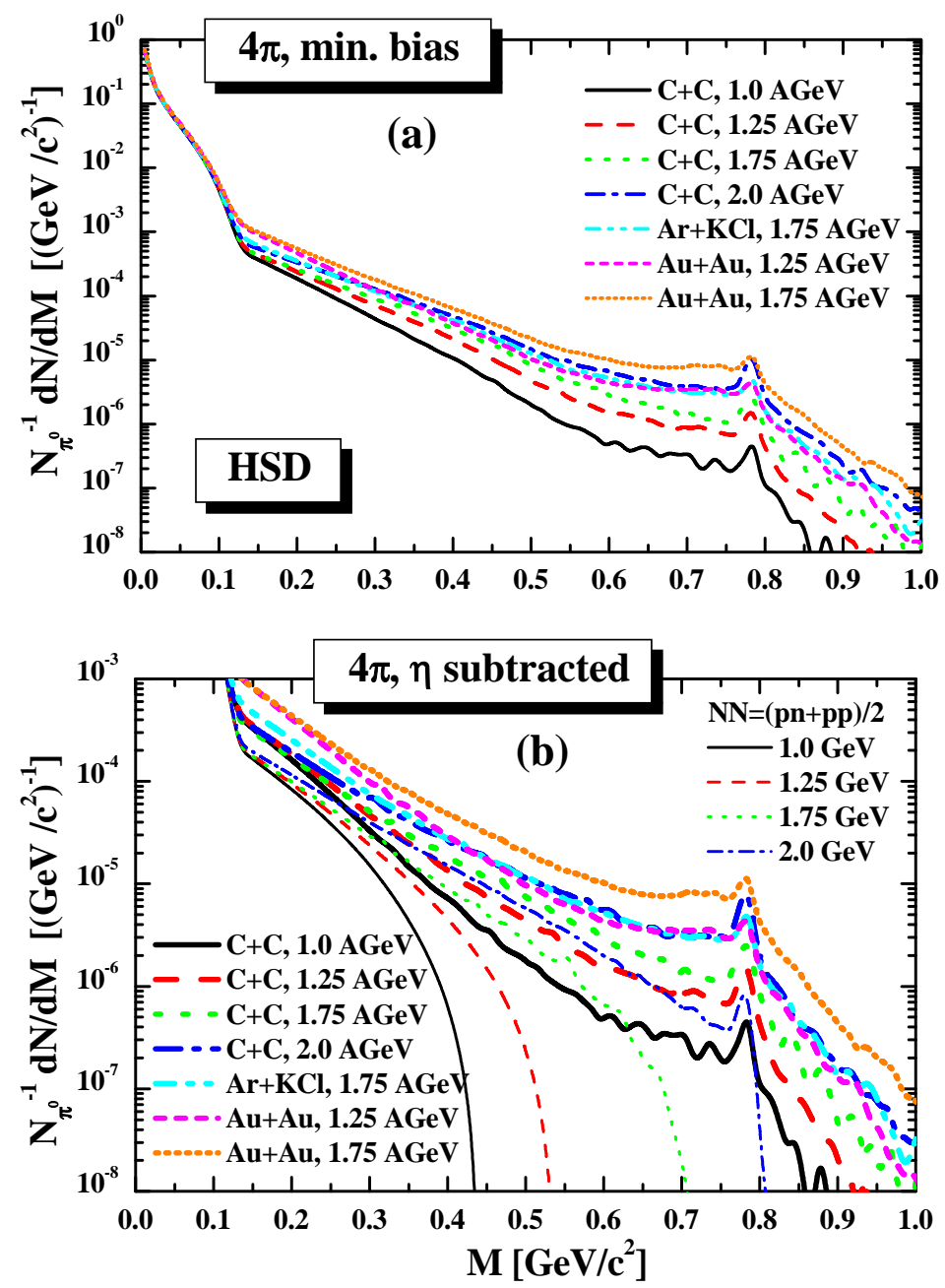

FIG. 31: (Color online) The invariant mass differential dilepton spectra - normalized to the $\pi^{0}$ multiplicity- obtained in HSD calculations for the minimal bias $\mathrm{C}+\mathrm{C}, \mathrm{Ar}+\mathrm{KCl}, \mathrm{Au}+\mathrm{Au}$ collisions and for the isospin-averaged reference spectra $N N=$ $(p n+p p) / 2$ at 1.0, 1.25, 1.75, $2.0 A \mathrm{GeV}$ in $4 \pi$ acceptance. The upper plot (a) corresponds to the total dilepton $A+A$ spectra whereas the lower plot (b) shows the dilepton spectra after $\eta$ Dalitz yield subtraction. The thick lines on the lower plot stand for the $A+A$ dilepton yields whereas the thin lines show the $N N$ spectra at the same energy.

Fig. 32 presents the ratio $\left(1 / N_{\pi^{0}}^{A A} d N^{A A} / d M\right) /\left(1 / N_{\pi^{0}}^{N N} d N^{N N} / d M\right)$ of the mass differential dilepton spectra normalized to the $\pi^{0}$ multiplicities - obtained in HSD calculations. Displayed are the ratios of minimal bias C+C, $\mathrm{Ar}+\mathrm{KCl}, \mathrm{Au}+\mathrm{Au}$ collisions and of the isospin-averaged reference spectra $N N=(p n+p p) / 2$ at the same energy. The lower plot depicts the same ratios but for the dilepton spectra after $\eta$ Dalitz yield subtraction. Clearly we see a quite complex structure. We start with the energy dependence of the ratio which decreases with energy. Including the $\eta$ production this can be clearly seen by comparing the Au+Au collisions at 1.75 and at $1.25 A \mathrm{GeV}$ as well as by comparing the $\mathrm{C}+\mathrm{C}$ system at different energies; $\eta$ subtraction modifies some details but does not change the tendency. It is also obvious that the ratio increases with the system size. The ratio for $\mathrm{Au}+\mathrm{Au}$ at $1.25 \mathrm{AGeV}$ is about 4.5, that of $\mathrm{C}+\mathrm{C}$ at the same energy around 2.5. We study now the origin of this enhancement in detail.

In Fig. 33 we display the enhancement factor in heavy-ion collisions for two different processes: Bremsstrahlung and $\Delta$ Dalitz decay. We show the ratio $\left(1 / N_{\pi^{0}}^{A A} d N^{A A} / d M\right) /\left(1 / N_{\pi^{0}}^{N N} d N^{N N} / d M\right)$ of the dilepton yield from HSD calculations of the minimal bias $A+A$ collisions: $\mathrm{C}+\mathrm{C}, \mathrm{Ar}+\mathrm{KCl}, \mathrm{Au}+\mathrm{Au}$ and of the isospin-averaged reference spectra $N N=(p n+p p) / 2$ at the same energy. The upper part shows the contribution from bremsstrahlung, the lower part that from the $\Delta$ Dalitz decay.

We do not expect that bremsstrahlung, one of the dominant sources at beam energies around $1 \mathrm{AGeV}$, scales with the number of pions; therefore the ratio should deviate from one. It has to be systematically larger than one due to multiple collisions of incoming nucleons in heavy-ion collisions. We see that the ratio depends on the mass but little 

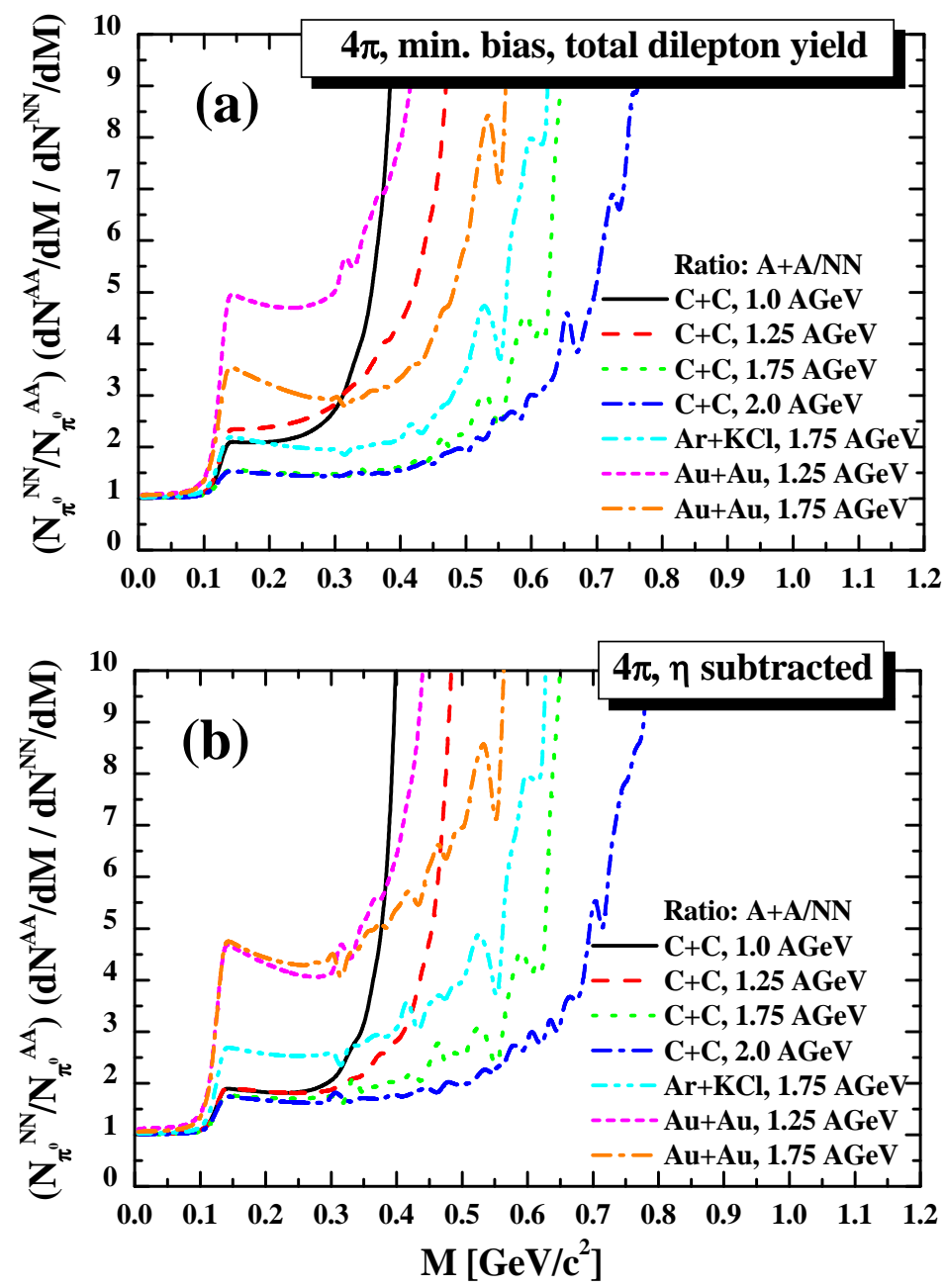

FIG. 32: (Color online) Upper plot (a): The ratio $\left(1 / N_{\pi^{0}}^{A A} d N^{A A} / d M\right) /\left(1 / N_{\pi^{0}}^{N N} d N^{N N} / d M\right)$ of the invariant mass differential dilepton $4 \pi$ spectra - normalized to the $\pi^{0}$ multiplicity - from HSD calculations for minimal bias $A+A$ collisions: We display $\mathrm{C}+\mathrm{C}, \mathrm{Ar}+\mathrm{KCl}, \mathrm{Au}+\mathrm{Au}$ collisions in comparison to the isospin-averaged reference spectra $N N=(p n+p p) / 2$ at $1.0,1.25,1.75$, $2.0 \mathrm{AGeV}$. Lower plot (b): the same ratios but for the dilepton spectra after $\eta$ Dalitz yield subtraction.

on the energy of the system. In $\mathrm{Au}+\mathrm{Au}$ collisions where the number of elementary collisions is large the enhancement can reach a factor of 3 . At higher energies the bremsstrahlung contribution is not really settled because there are no reliable calculations for the elastic and inelastic elementary channels.

The other dominant source for dilepton production at beam energies around $1 A \mathrm{GeV}$ is $\Delta$ Dalitz decay. One may assume that the $\Delta$ Dalitz decay scales with the number of pions because the relative ratio is given by the branching ratio but this is not the case. First of all, we are here in a threshold region where the Fermi momentum only can lead to a substantial enhancement of the production. Secondly, pions from $\Delta$ decay can be reabsorbed by nucleons and can form again a $\Delta$ which may later disappear in a $\Delta N \rightarrow N N$ collisions. This process is even important in systems as small as $\mathrm{C}+\mathrm{C}$. Dileptons, on the contrary, cannot be reabsorbed and are seen in the detector. Table I shows quantitatively the consequences of these processes for reactions at $1 A \mathrm{GeV}$. We compare there the pion and dilepton yield for $\mathrm{C}+\mathrm{C}$ and $\mathrm{Au}+\mathrm{Au}$ for different conditions. If there is neither a Fermi momentum (Fermi m.) nor meson absorption on baryons $\left(\mathrm{mB}\right.$ col.) the ratio of $\pi^{0}$ 's to dileptions corresponds to the branching ratios and the enhancement factor (last column) is one, independent of the system size of the heavy-ion reaction. The Fermi motion alone increases the pion yield ( $6^{\text {th }}$ column) as well as the dilepton yield ( $7^{\text {th }}$ column) by almost a factor of two. Because in the ratio displayed in Fig. 33 one divides by the number of pions this ratio remains one for small invariant masses whereas the Fermi motion makes the ratio explode for invariant masses close to the phase space boundary. Meson-baryon interactions ( $\mathrm{mB}$ coll) lower the number of pions in heavy-ion collisions, by $15 \%$ in $\mathrm{C}+\mathrm{C}$ collisions 
TABLE I: Ratio of $\pi^{0}$ mesons and the integrated dilepton yield $\left(N\left(\Delta \rightarrow e^{+} e^{-}\right)=\int d M \frac{d N\left(\Delta \rightarrow e^{+} e^{-}\right)}{d M}\right)$ from $\Delta$ Dalitz decays for $\mathrm{C}+\mathrm{C}$ and $\mathrm{Au}+\mathrm{Au}$ at $\mathrm{b}=0.5 \mathrm{fm}$ and $1 \mathrm{AGeV}$ and that from the 'elementary' $N N$ reactions for different scenarios: with/without Fermi motion ('Fermi m.'), with/without secondary meson-baryon collisions ('mB col.')

\begin{tabular}{|c|c|c|c|c|c|c|c|}
\hline 1 & 2 & 3 & 4 & 5 & 6 & 7 & 8 \\
\hline Fermi m. & $\mathrm{mB}$ col. & system & $N\left(\pi^{0}\right)$ & $N\left(\Delta \rightarrow e^{+} e^{-}\right)$ & $R\left(\pi^{0}\right)=\frac{N^{A A}\left(\pi^{0}\right)}{N^{N N}\left(\pi^{0}\right)}$ & $R\left(e^{+} e^{-}\right)=\frac{N^{A A}\left(\Delta \rightarrow e^{+} e^{-}\right)}{N^{N N}\left(\Delta \rightarrow e^{+} e^{-}\right)}$ & $\frac{R\left(e^{+} e^{-}\right)}{R\left(\pi^{0}\right)}=\frac{(7)}{(6)}$ \\
\hline- & - & $\mathrm{CC}$ & 0.743 & $0.565 \times 10^{-4}$ & 6.74 & 5.56 & 0.83 \\
- & - & $\mathrm{AuAu}$ & 18.76 & $1.688 \times 10^{-3}$ & 170.08 & 166.3 & 0.98 \\
\hline+ & - & $\mathrm{CC}$ & 1.407 & $1.16 \times 10^{-4}$ & 12.76 & 11.42 & 0.89 \\
+ & - & $\mathrm{AuAu}$ & 31.07 & $2.75 \times 10^{-3}$ & 281.69 & 270.93 & 0.97 \\
\hline- & + & $\mathrm{CC}$ & 0.633 & $0.86 \times 10^{-4}$ & 5.74 & 8.47 & 1.47 \\
- & + & $\mathrm{AuAu}$ & 10.75 & $3.45 \times 10^{-3}$ & 97.46 & 339.8 & 3.49 \\
\hline+ & + & $\mathrm{CC}$ & 1.07 & $1.77 \times 10^{-4}$ & 9.70 & 17.44 & 1.80 \\
+ & + & $\mathrm{AuAu}$ & 16.62 & $6.32 \times 10^{-3}$ & 150.68 & 622.66 & 4.13 \\
\hline
\end{tabular}

and by $47 \%$ in $\mathrm{Au}+\mathrm{Au}$ collisions because they can lead to a disappearance of the pions if the $\pi N \rightarrow \Delta$ collision is followed by a $\Delta N \rightarrow N N$ collision. At the same time they enhance the dilepton yield because dileptons do not get reabsorbed and therefore every $\Delta$ which is produced contributes to the dilepton yield. The meson-baryon interactions are therefore the reason that dileptons behave differently than pions. This cycle of $\Delta$ production, $\Delta$ decay and $\pi$ reabsorption in $\pi N \rightarrow \Delta$ collisions, which leads in heavy system to the creation of several generations of $\Delta$ 's, has been studied already 20 years ago as one of the key elements to the pion dynamics in heavy-ion collision which allows the pions to equilibrate with the system and to serve as a measure of the number of participants [87]. The last two lines of Table I show that the pion absorption enhances the dilepton production as compared to the pions by a factor of about 1.5-1.7 in $\mathrm{C}+\mathrm{C}$ collisions and by a factor of 3.5-4.1 for $\mathrm{Au}+\mathrm{Au}$ collisions, i.e. the enhancement grows with the size of the system.

The system size effect is demonstrated explicitly in Fig. 34 the left plot shows the ratio of the mass differential dilepton spectra $\left(1 / N_{\pi^{0}}^{A A} d N^{A A} / d M\right) /\left(1 / N_{\pi^{0}}^{N N} d N^{N N} / d M\right)$ - normalized to the $\pi^{0}$ multiplicity and after $\eta$ Dalitz yield subtraction - from HSD calculations for the minimal bias $\mathrm{C}+\mathrm{C}, \mathrm{Ar}+\mathrm{KCl}, \mathrm{Cr}+\mathrm{Cr}, \mathrm{Ti}+\mathrm{Pb}, \mathrm{Au}+\mathrm{Au}$ collisions and of the isospin-averaged reference spectra $N N=(p n+p p) / 2$ at $1.75 \mathrm{AGeV}$. The right plot shows the same but for the $\Delta$ Dalitz decay contributions only. We see also here that the different ratios are separated by a factor which is (almost) independent of invariant mass and depends basically on the size of the colliding nuclei since the effect of multiple $\Delta$ regeneration increases with the atomic number of the colliding ions.

Thus, the dilepton enhancement observed in Fig. 32 (and hence also in the experimental spectra) is due to bremsstrahlung and due to the $\Delta$ dynamics in the medium. Both are not related to collective effects like the inmedium modifications of spectral functions but are a mere consequence of the presence of other nucleons in the nuclei. They also appear if no potential but only collisional interactions between the nucleons exist. This effect grows with the nuclear size which is directly related to an increase of the high baryon density phase from light to heavy-ion collisions. This is demonstrated in Fig. 35 which shows the time evolution of the baryon density from HSD in the central cell $\rho(0,0,0, t)$ in units of the normal nuclear density $\rho_{0}=0.168 \mathrm{fm}^{-3}$ for central $(b=0 \mathrm{fm}) \mathrm{Au}+\mathrm{Au}$ (left plot) and $\mathrm{Ar}+\mathrm{KCl}$ (right plot) at different energies - 1.25, 1.7, 2.0 and 3.5 AGeV. By comparing the $\mathrm{Ar}+\mathrm{KCl}$ and $\mathrm{Au}+\mathrm{Au}$ density profiles one sees that the maximum density reached in the central cell is approximately the same in both cases - up to $3 \rho_{0}$ and only slightly grows with increasing energy. However, the high baryon density phase for the heavy $\mathrm{Au}+\mathrm{Au}$ nuclei collisions is much longer than for the intermediate $\mathrm{Ar}+\mathrm{KCl}$ system which implies a longer reaction time and a stronger influence of secondary reactions on observables as discussed above.

\section{In-medium effects in vector meson production}

Now we come to the question - how the in-medium effects in vector meson production can influence the ratios. The dilepton spectra for $p+N b$ at $3.5 \mathrm{AGeV}, \mathrm{C}+\mathrm{C}$ at 1.0,2.0 $\mathrm{AGeV}$ and for $\mathrm{Ar}+\mathrm{KCl}$ at $1.75 \mathrm{AGeV}$ within the collisional broadening scenario for the vector meson spectral functions have been already presented in Sections III and IV (cf. Figs. 13]14[16]18) in comparison to the HADES data as well as our predictions for $\mathrm{Au}+\mathrm{Au}$ at $1.25 \mathrm{AGeV}$ (cf. Fig. 21).

In Fig. 36 we display for reactions at $1.70 A \mathrm{GeV}$ the system size dependence of the $4 \pi$ mass differential dilepton spectra - normalized to the $\pi^{0}$ multiplicity - from HSD calculations for minimal bias $A+A$ reactions. We display 

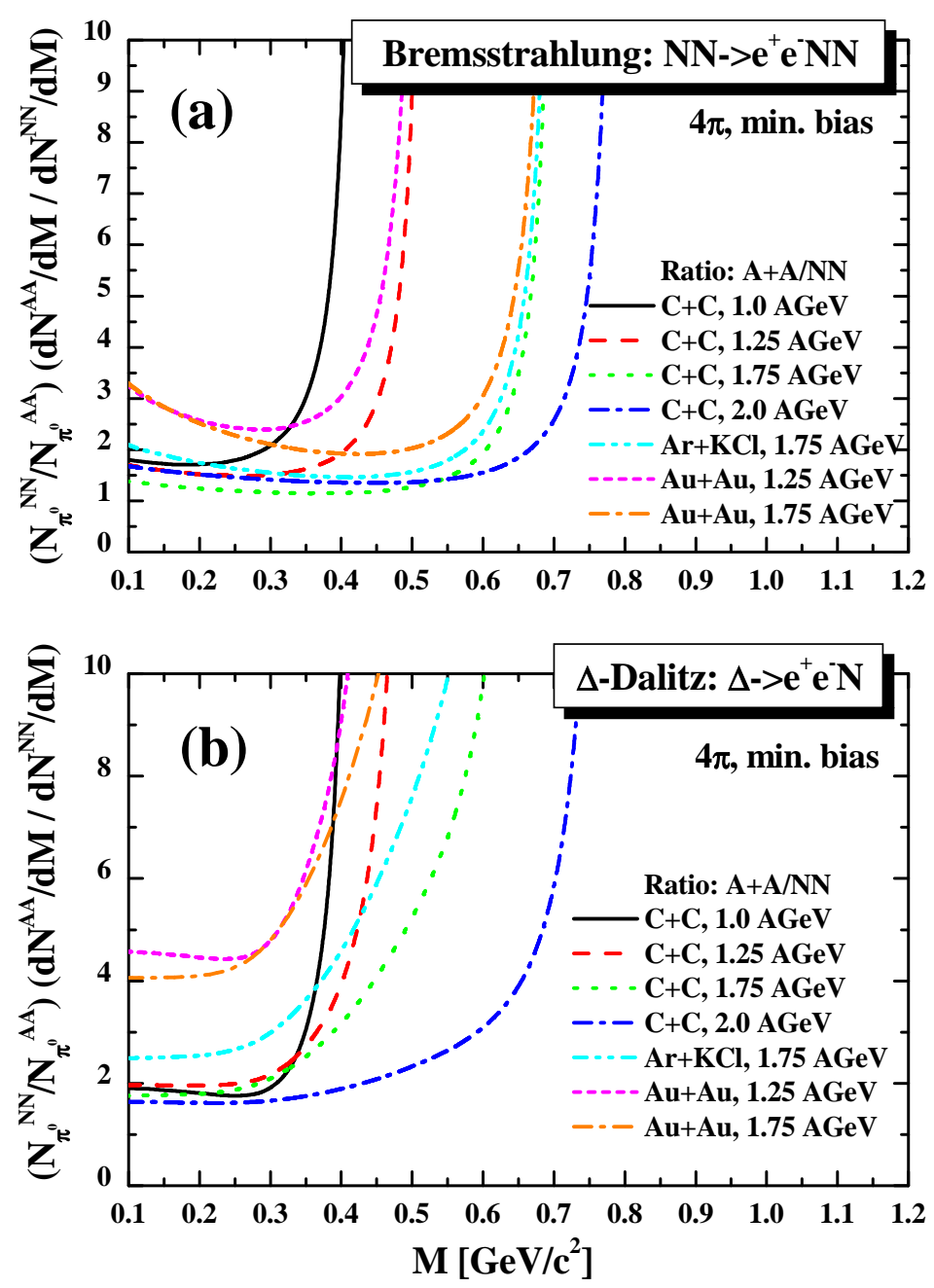

FIG. 33: (Color online) The ratio $\left(1 / N_{\pi^{0}}^{A A} d N^{A A} / d M\right) /\left(1 / N_{\pi^{0}}^{N N} d N^{N N} / d M\right)$ of the dilepton yield from the bremsstrahlung channel (upper part (a)) and $\Delta$ Dalitz decay (lower part (b)) - normalized to the multiplicity of $\pi^{0}$. We display HSD calculations for the ratio of the minimal bias $\mathrm{C}+\mathrm{C}, \mathrm{Ar}+\mathrm{KCl}, \mathrm{Au}+\mathrm{Au}$ collisions and the isospin-averaged reference spectra $N N=(p n+p p) / 2$ at the same energy.

the result for the symmetric $\mathrm{Cr}+\mathrm{Cr}$ and $\mathrm{Au}+\mathrm{Au}$ systems as well as for the asymmetric $\mathrm{Ti}+\mathrm{Pb}$ system. The solid lines stand for the 'no medium effects' scenario whereas the dashed lines show the dilepton yield for the 'collisional broadening' scenario. The lower plot is a 'zoom' of the upper one for the mass range $0.4<M<1.0 \mathrm{GeV}$. First of all we note the growth of the dilepton yield for $0.15 \leq M \leq 0.6 \mathrm{GeV}$ when going from the intermediate $\mathrm{Cr}+\mathrm{Cr}$ to the heavy system $\mathrm{Au}+\mathrm{Au}$. The larger the system mass, the more important is the aforementioned $\Delta$ reaction cycle and the more the dilepton production is enhanced as compared to pion production. As we have discussed already in Sections III and IV, for the collisional broadening scenario one sees clearly the influence of the larger width of the vector meson resonances (the peaks get smaller and broader).

What would be the consequence of this in-medium effect on the dilepton ratio of $A A$ spectra to the 'reference spectrum'? Would this observable yield information on the underlying dynamical processes? Previously we concentrated on the ratio $R(A A / N N)$ where the 'reference spectrum' is constructed as an average of $p p$ and $p n$ yields: $N N=(p p+p n) / 2$. However, such a ratio would not be well suited for studying in-medium effects in the vector meson mass region due to the limited open phase space in $N N$ collisions relative to $A A$ collisions - taken at the same energies - since the Fermi motion in $A A$ extends the kinematical limits, which leads to a fast rise of $R(A A / N N)$ at larger invariant masses $M$. Moreover, as has been discussed in Section III.A, there is a general problem with $N N$ as a 'reference spectrum' since experimentally $p n$ are usually quasi-free $p d$ reactions. For the beam energies discussed here, in the interesting invariant mass region, $M>0.5 \mathrm{GeV}$ there are no 'quasi-free' $p n$ collisions anymore but genuine 

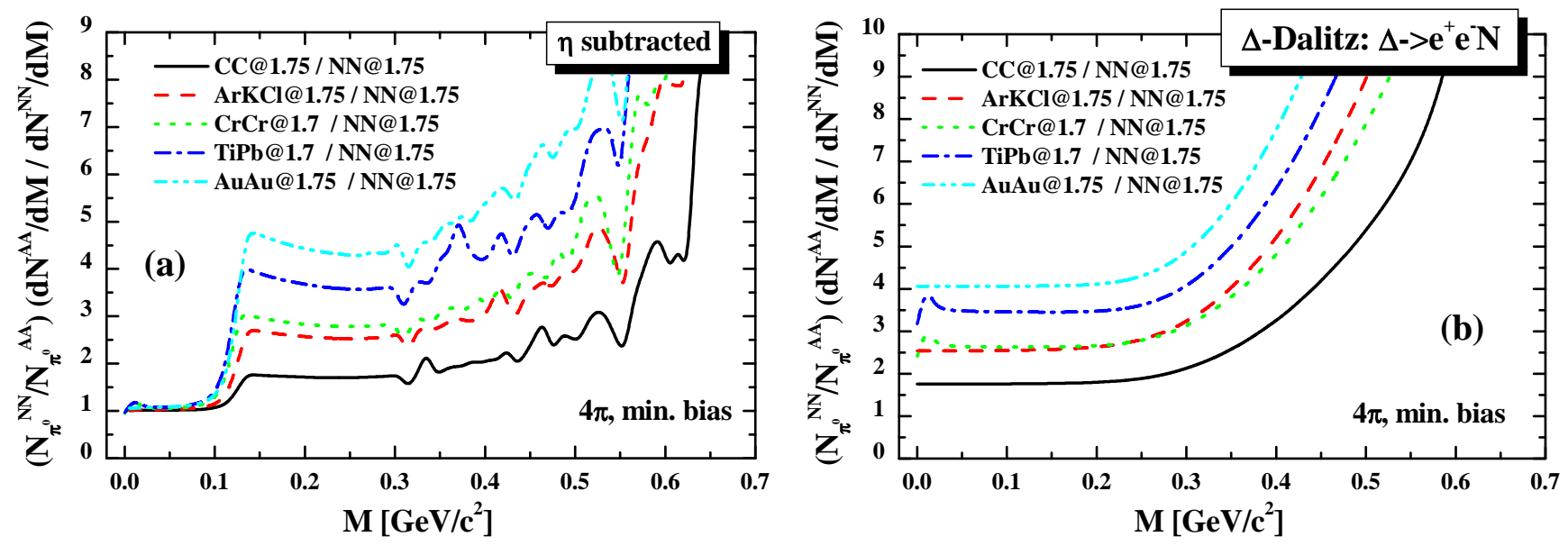

FIG. 34: (Color online) Left (a): The $4 \pi$ ratio $\left(1 / N_{\pi^{0}}^{A A} d N^{A A} / d M\right) /\left(1 / N_{\pi^{0}}^{N N} d N^{N N} / d M\right)$ of the mass differential dilepton spectra - normalized to the $\pi^{0}$ multiplicity and after $\eta$ Dalitz yield subtraction - from HSD calculations for the minimal bias $\mathrm{C}+\mathrm{C}, \mathrm{Ar}+\mathrm{KCl}, \mathrm{Cr}+\mathrm{Cr}, \mathrm{Ti}+\mathrm{Pb}$ and $\mathrm{Au}+\mathrm{Au}$ collisions to the isospin-averaged reference spectra $N N=(p n+p p) / 2$ at 1.75 $A \mathrm{GeV}$. Right (b): same as the left plot but for the $\Delta$ Dalitz decay contributions, only.
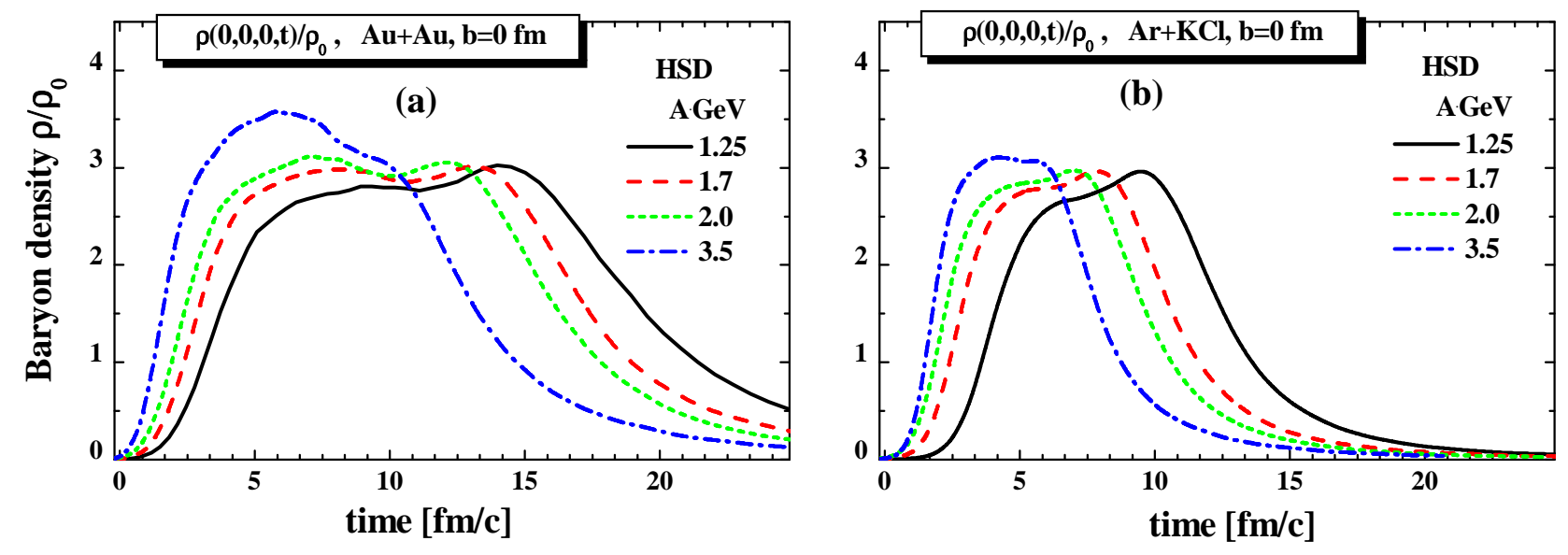

FIG. 35: (Color online) The time evolution of the baryon density from HSD in the central cell $\rho(0,0,0, t)$ in units of the normal nuclear density $\rho_{0}=0.168 \mathrm{fm}^{-3}$ for central $(b=0 \mathrm{fm}) \mathrm{Au}+\mathrm{Au}$ (left plot $\left.(\mathrm{a})\right)$ and $\mathrm{Ar}+\mathrm{KCl}$ (right plot (b)) at different energies - 1.25, 1.7, 2.0 and $3.5 \mathrm{AGeV}$.

three-body $p d$ collisions.

Alternatively, the in-medium enhancement can be studied by comparing the yield of a heavy system to that of a light system. Fig 37 displays for a beam energy of $1.7 A \mathrm{GeV}$ the ratio of the invariant mass differential dilepton spectra for intermediate $\mathrm{Cr}+\mathrm{Cr}$ and heavy $\mathrm{Au}+\mathrm{Au}$ nuclei and of the light nuclei $\mathrm{C}+\mathrm{C}$, which is chosen as a 'reference spectrum'. We study two scenarios - the 'no medium effects' and the 'collisional broading' scenario. One clearly sees that the enhancement for $M \leq 0.5 \mathrm{GeV}$ due to the multiple $\Delta$ production and bremsstrahlung persists when one compares collisions of heavy and light nuclei and can become as large as a factor of two. Thus, $\mathrm{C}+\mathrm{C}$ collisions can also be used as 'reference spectra' to study such nuclear effects. Moreover, we observe as well that the difference between the two scenarios is small for low invariant masses and becomes only noticeable at invariant masses close to the $\rho$ mass. However, even there the differences remain moderate. Therefore high precision data are required to study the question whether vector mesons are modified by the strongly interacting medium in this energy region. On the other hand the ratio for $\mathrm{AuAu} / \mathrm{CC}$ grows much faster (for both - the no medium and the in-medium scenario) than for $\mathrm{CrCr} / \mathrm{CC}$. This is due to the enhancement of the vector meson productions by secondary meson-baryon and meson-meson interactions in heavy system relative to the light system. This effect is hence easy to observe experimentally. 

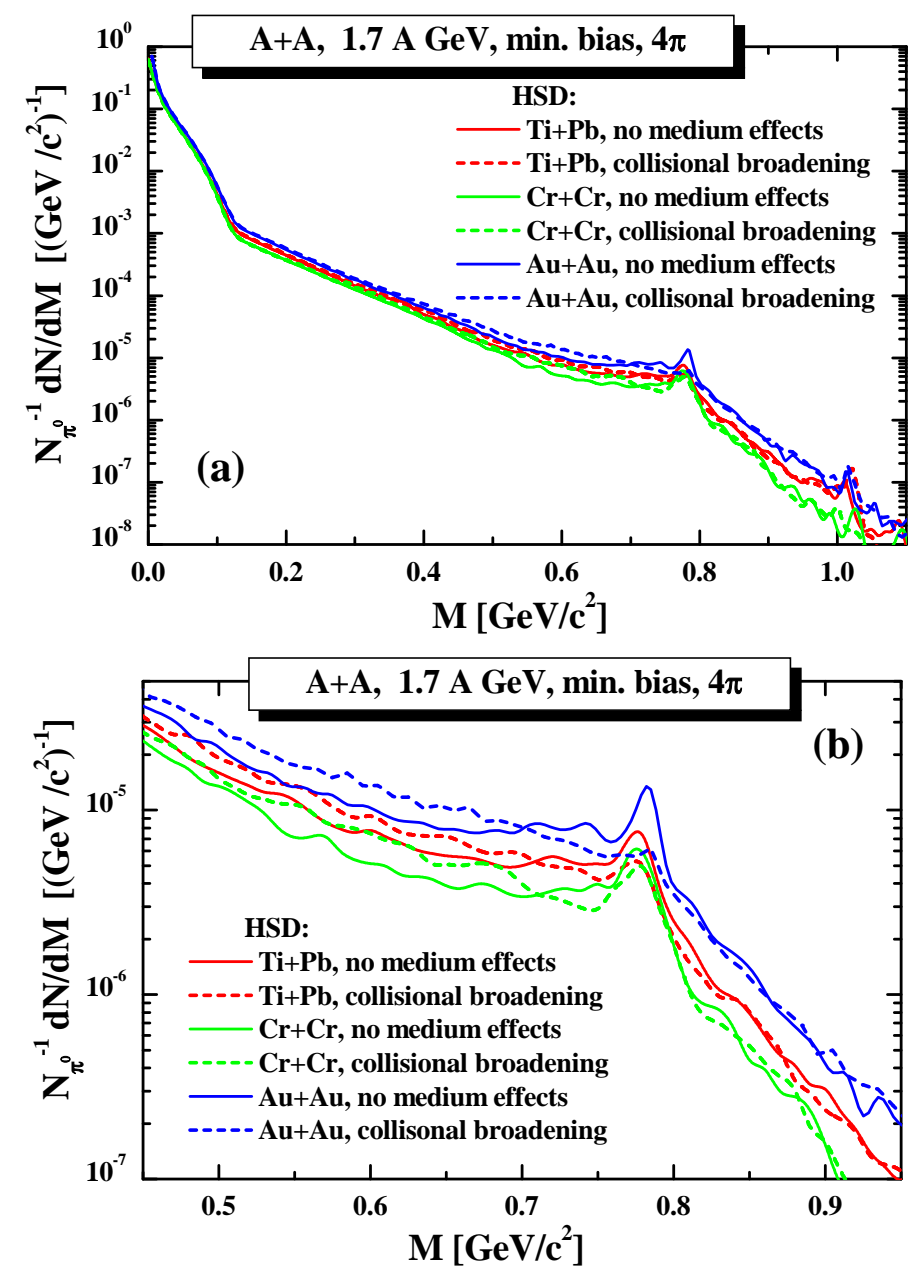

FIG. 36: (Color online) The $4 \pi$ mass differential dilepton spectra - normalized to the $\pi^{0}$ multiplicity - from HSD calculations for minimal bias $\mathrm{Ti}+\mathrm{Pb}, \mathrm{Cr}+\mathrm{Cr}$ and $\mathrm{Au}+\mathrm{Au}$ collisions at $1.7 \mathrm{AGeV}$. The solid lines stand for the 'no medium effects' scenario whereas the dashed lines show the dilepton yield for the 'collisional broadening' scenario. The lower plot (b) is a 'zoom' of the upper plot (a) for the mass range $0.4<M<1.0 \mathrm{GeV}$.

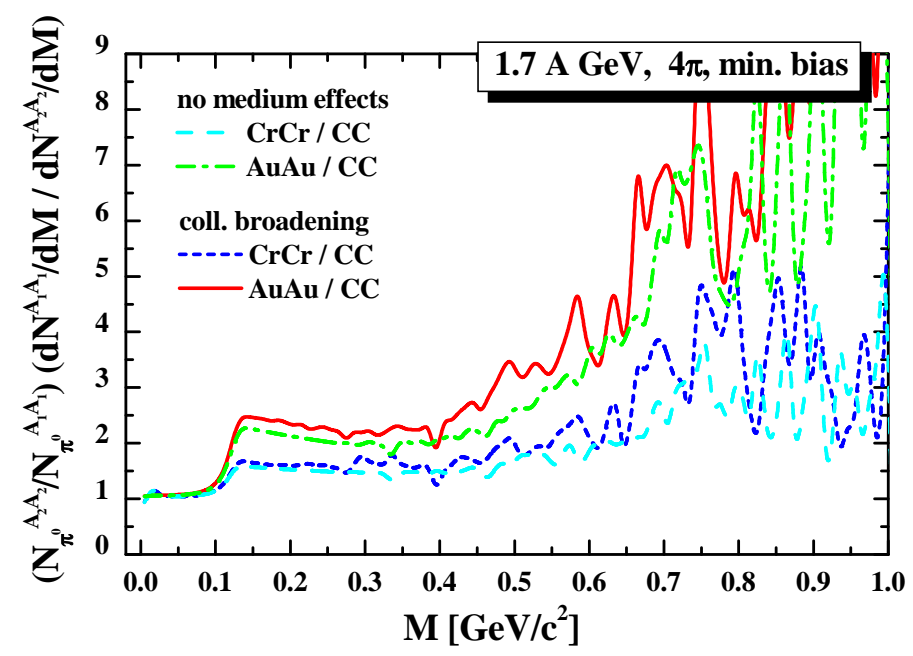

FIG. 37: (Color online) The $4 \pi$ ratio $\left(1 / N_{\pi^{0}}^{A-2 A_{2}} d N^{A_{2} A-2} / d M\right) /\left(1 / N_{\pi^{0}}^{A_{1} A_{1}} d N^{A_{1} A_{1}} / d M\right)$ of the mass differential dilepton spectra - normalized to the $\pi^{0}$ multiplicity - from HSD calculations for minimal bias $\mathrm{Au}+\mathrm{Au}(\mathrm{Cr}+\mathrm{Cr})$ collisions and $\mathrm{C}+\mathrm{C}$ collisions. This ratio is displaed for the 'no medium effects' and for the 'collisional broadening' scenario. 


\section{UNCERTAINTIES DUE TO DIFFERENT ASSUMPTIONS IN THE TRANSPORT MODELS}

In this section we discuss the different assumptions in different transport approaches due to the lack of experimental information and theoretical knowledge and the consequences for the prediction of these approaches. The uncertainties related to the production cross sections in elementary reactions have been addressed already in Section II. There are, however, other sources of uncertainties, in particular for the dilepton production by $\Delta$ Dalitz decay - the lack of knowledge of the electromagnetic decay width of the $\Delta$ resonance, of the mass distribution of the $\Delta$ resonance in elementary $N N$ collisions and of its total decay width as well as different assumptions on how the total decay width is related to the $\Delta$ life time.

\section{A. Electromagnetic decay width of $\Delta$ resonance}

The differential electromagnetic decay width of a $\Delta$ resonance into dileptons of an invariant mass $\mathrm{M}, \Delta \rightarrow N e^{+} e^{-}$, can be related to the Delta decay into a nucleon and a virtual photon, $\Delta \rightarrow N \gamma^{*}$, by (cf. [53]):

$$
\frac{d \Gamma}{d M}^{\Delta \rightarrow N l+l-}(M)=\frac{2 \alpha}{3 \pi} \frac{\Gamma^{\Delta \rightarrow N \gamma^{*}}\left(M, M_{\Delta}\right)}{M},
$$

where $\alpha=1 / 137$ and $M_{\Delta}$ is the current mass of the $\Delta$-resonance. Unfortunately there is no direct measurement of the $\Delta \rightarrow N \gamma^{*}$ width and starting from the pioneering work of Jones and Scadron [89] there is a series of different models [30, 53, 90, 91]. In the present versions of the HSD, IQMD and UrQMD transport approaches the "Wolf" model is employed for the electromagnetic decay width [53] :

$$
\begin{aligned}
& \Gamma^{\Delta \rightarrow N \gamma^{*}}\left(M, M_{\Delta}\right)=\frac{\lambda^{1 / 2}\left(M^{2}, m_{N}^{2}, M_{\Delta}^{2}\right)}{16 \pi M_{\Delta}^{2}} \cdot m_{N} \cdot\left[2 m_{T}\left(M, M_{\Delta}\right)+m_{L}\left(M, M_{\Delta}\right)\right] \\
& m_{L}\left(M, M_{\Delta}\right)=(e f g)^{2} \frac{M_{\Delta}^{2}}{9 m_{N}} M^{2} \cdot 4\left(M_{\Delta}-m_{N}-q_{0}\right), \quad e^{2}=4 \pi \alpha, \quad g=5.44 \\
& m_{T}\left(M, M_{\Delta}\right)=(e f g)^{2} \frac{M_{\Delta}^{2}}{9 m_{N}}\left[q_{0}^{2}\left(5 M_{\Delta}-3\left(q_{0}+m_{N}\right)\right)-M^{2}\left(M_{\Delta}+m_{N}+q_{0}\right)\right] \\
& f=-1.5 \frac{M_{\Delta}+m_{N}}{m_{N}\left(\left(m_{N}+M_{\Delta}\right)^{2}-M^{2}\right)} \\
& q_{0}=\left(M^{2}+p_{f}^{2}\right)^{1 / 2} \\
& p_{f}^{2}=\frac{\left(M_{\Delta}^{2}-\left(m_{N}+M^{2}\right)\left(M_{\Delta}^{2}-\left(m_{N}-M\right)^{2}\right)\right.}{4 M_{\Delta}^{2}} \\
& \lambda\left(M^{2}, m_{N}^{2}, M_{\Delta}^{2}\right)=M^{4}+m_{N}^{4}+M_{\Delta}^{4}-2\left(M^{2} m_{N}^{2}+M^{2} M_{\Delta}^{2}+m_{N}^{2} M_{\Delta}^{2}\right) .
\end{aligned}
$$

There is a variety of models for the electromagnetic decay width of $\Delta$ resonance to dileptons $\Delta \rightarrow N e^{+} e^{-}-$cf. [30, 53, 90, 91]. Figure 38 shows $\Gamma^{\Delta \rightarrow N}$ e+e- $\left(M, M_{\Delta}\right)$ for different models denoted as "Wolf" [53], "Zetenyi" [91], "Ernst" [30] and "Krivoruchenko" [90] and for three different Delta masses - $1.232 \mathrm{GeV}$ (a), $1.5 \mathrm{GeV}$ (b) and 1.8 $\mathrm{GeV}$ (c). One can see that in the low mass region, i.e. around the $\Delta$ pole mass 1.232 , all approaches give similar results whereas with increasing $M_{\Delta}$ the differences grow. The models "Wolf" and "Ernst" lead to a similar dilepton yield which is, however, up to a factor of 3 higher then that from the models "Krivoruchenko" and "Zatenyi". This introduces a systematic error for the prediction of the dilepton yield for large mass dileptons.

\section{B. Total decay width and the life-time of $\Delta$ resonance}

The population of high mass Delta's in NN reactions depends on the shape of the differential mass distribution which is given by the $\Delta$ spectral function. The spectral function of a $\Delta$ resonance of mass $M_{\Delta}$ is usually assumed to be of the relativistic Breit-Wigner form:

$$
A_{\Delta}\left(M_{\Delta}\right)=C_{1} \cdot \frac{2}{\pi} \frac{M_{\Delta}^{2} \Gamma_{\Delta}^{t o t}\left(M_{\Delta}\right)}{\left(M_{\Delta}^{2}-M_{\Delta 0}^{2}\right)^{2}+\left(M_{\Delta} \Gamma_{\Delta}^{t o t}\left(M_{\Delta}\right)\right)^{2}} .
$$



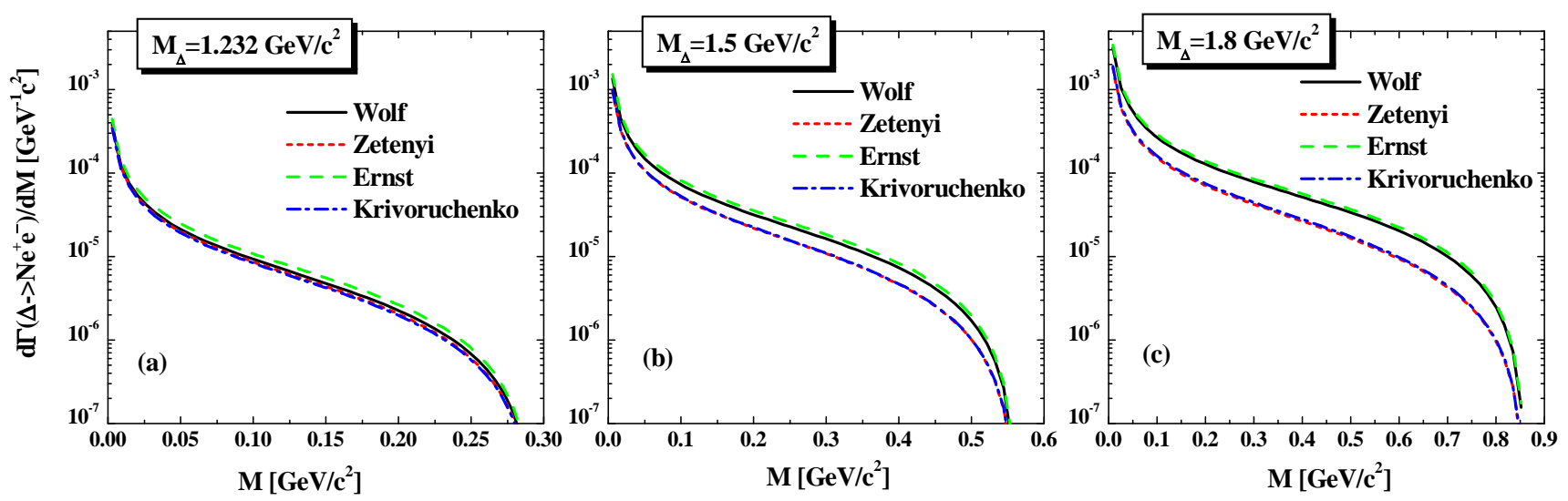

FIG. 38: (Color online) The electromagnetic decay width of $\Delta$ resonance to dileptons $\Delta \rightarrow N e^{+} e^{-}$for different models denoted as "Wolf" [53], "Zetenyi" [91], "Ernst" 30] and "Krivoruchenko" 90] for different Delta masses of 1.232 GeV (a), $1.5 \mathrm{GeV}$ (b) and $1.8 \mathrm{GeV}(\mathrm{c})$.

with $M_{\Delta 0}$ being the pole mass of the $\Delta$. The factor $C_{1}$ is fixed by the normalization condition:

$$
\int_{M_{\text {min }}}^{M_{l i m}} A_{\Delta}\left(M_{\Delta}\right) d M_{\Delta}=1
$$

where $M_{l i m}=2 \mathrm{GeV}$ is chosen as an upper limit for the numerical integration. The lower limit for the vacuum spectral function corresponds to the nucleon-pion decay, $M_{\min }=m_{\pi}+m_{N}$. In $N N$ collisions the Deltas can be populated up to the $M_{\max }=\sqrt{s}-m_{N}$ and hence the available part of spectral function is defined by the beam energy.

The shape of spectral function (and correspondingly the production of high mass Delta's) depends strongly on the total width $\Gamma_{\Delta}^{t o t}$. Due to the lack of experimental information this total width has to be assumed and different parametrizations exist.

For the present HSD calculations we adopt the "Monitz" model [92] (cf. also Ref. [53]) :

$$
\begin{aligned}
\Gamma_{\Delta}^{t o t}\left(M_{\Delta}\right)= & \Gamma_{R} \frac{M_{\Delta 0}}{M_{\Delta}} \cdot\left(\frac{q}{q_{r}}\right)^{3} \cdot F^{2}(q) \\
& q^{2}=\frac{\left(M_{\Delta}^{2}-\left(m_{N}+m_{\pi}\right)^{2}\right)\left(M_{\Delta}^{2}-\left(m_{N}-m_{\pi}\right)^{2}\right)}{4 M_{\Delta}^{2}} \\
& \Gamma_{R}=0.11 \mathrm{GeV}, \quad M_{\Delta 0}=1.232 \mathrm{GeV} \\
& F(q)=\frac{\beta_{r}^{2}+q_{r}^{2}}{\beta_{r}^{2}+q^{2}} \\
& q_{r}^{2}=0.051936, \quad \beta_{r}^{2}=0.09
\end{aligned}
$$

In the UrQMD model one employs the "Bass" parametrization 71] which differs from the "Monitz" model (12) by the formfactor (13):

$$
F_{B}(q)=1.2 \frac{\tilde{\beta}_{r}^{2}}{\tilde{\beta}_{r}^{2}+q^{2}}, \quad \tilde{\beta}_{r}^{2}=q_{r}^{2} / 0.2
$$

In the left part (a) of Fig. 39 we show the mass dependence of the total width $\Gamma_{\Delta}^{\text {tot }}\left(M_{\Delta}\right)$ from different models: "Const" - a constant width $\Gamma_{\Delta}^{\text {tot }}=0.12 \mathrm{GeV}$, "Monitz" - from Eq. 12 (cf. [92]), "Bass" - from [14 (cf. 71]) as well as the parametrization used in the IQMD model [73, 74] denoted as "IQMD". We observe substantial differences between the models, especially for large mass $\Delta$. These differences become more important at higher energies. For lower energies, especially for the $1 \mathrm{AGeV}$ data, phase space limits the $\Delta$ masses to $M_{\Delta}<1.4 \mathrm{GeV}$.

The total decay width is related to the life time of the resonances - an other important quantity for the transport approaches - by

$$
\tau_{\Delta}\left(M_{\Delta}\right)=\frac{\hbar c}{\Gamma_{\Delta}^{t o t}\left(M_{\Delta}\right)}
$$



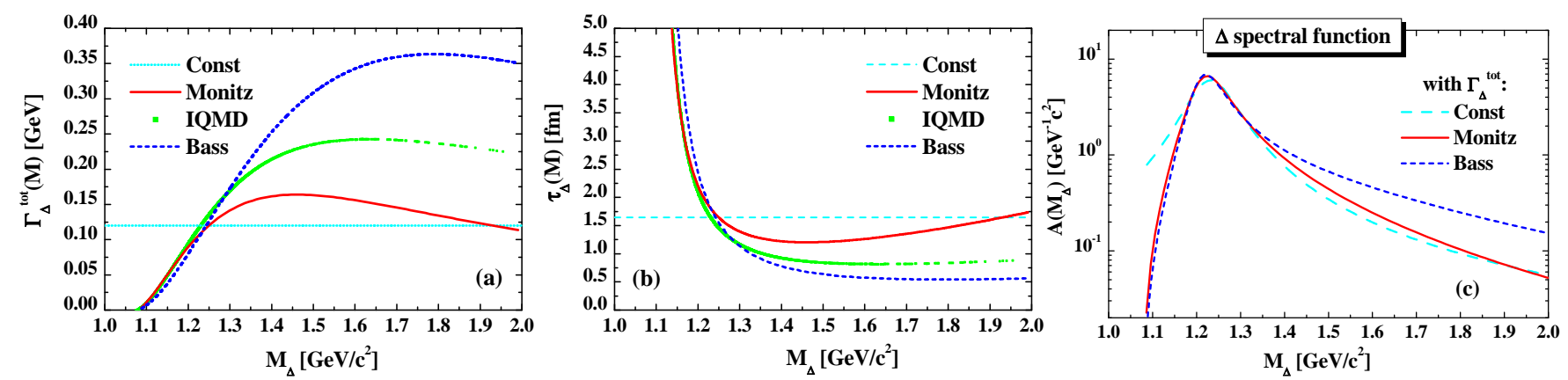

FIG. 39: (Color online) The mass dependence of the total width $\Gamma_{\Delta}^{\text {tot }}\left(M_{\Delta}\right)$ (left plot (a)), life time (middle plot (b)) and the spectral function (right plot (c)) from different models: "Const" - the constant width $\Gamma_{\Delta 0}^{t o t}=0.12 \mathrm{GeV}$, "Monitz" - from Eq. 12 (cf. [92]), "Bass" - from 14 (cf. 71]), "IQMD" - from [73, 74].

The lifetime as a function of $M_{\Delta}$ is illustrated in the middle part (b) of Fig. 39, The lifetime of large mass $\Delta$ is in the "Bass" parametrization up to three times lower than in the Monitz parametrization. $\Delta$ 's of such a high mass are rare, however, as can be see from the right part (c) of Fig. 39 which shows the mass dependence of the spectral function for different parametrizations of the width.

\section{Consequences for the dilepton yield}

Now we show how the uncertainties in the modelling of the total $\Delta$ width and of the electromagnetic decay width affect the final results for the dilepton yield.

\section{Convolution model}

We start out with a simple example: the dilepton yield from the $\Delta$ Dalitz decay is a convolution of the mass distribution of the $\Delta$ resonances - which we take for our model study to be defined by the spectral function $A_{\Delta}\left(M_{\Delta}\right)$ (Eq.(10) - and the $\Delta$ mass dependent branching ratio for the electromagnetic decay into dileptons which is defined as a ratio of electromagnetic partial width $\frac{d \Gamma}{d M}^{\Delta \rightarrow N e^{+} e^{-}}\left(M, M_{\Delta}\right)$ and the total width $\Gamma_{\Delta}^{t o t}\left(M_{\Delta}\right)$ :

$$
\begin{aligned}
& \frac{d N^{e^{+} e^{-}}}{d M}(M)=\int d M_{\Delta} \cdot A_{\Delta}\left(M_{\Delta}\right) \cdot \frac{d \Gamma}{d M} \stackrel{\Delta \rightarrow N e^{+} e^{-}}{\left(M, M_{\Delta}\right)} \cdot \frac{1}{\Gamma_{\Delta}^{t o t}\left(M_{\Delta}\right)} \\
& =\int d M_{\Delta} \cdot A_{\Delta}\left(M_{\Delta}\right) \cdot \frac{d \Gamma}{d M} \stackrel{\Delta \rightarrow N e^{+} e^{-}}{\left(M, M_{\Delta}\right) \cdot \tau_{\Delta}\left(M_{\Delta}\right) .}
\end{aligned}
$$

where the expression (16) has been re-written in terms of the $\Delta$ life time using relation (15).

In the left side (a) of Fig. 40 we show the dilepton yield for two different assumptions for $\Gamma_{\Delta}^{t o t}\left(M_{\Delta}\right)$ and for $\left.\frac{d \Gamma}{d M}{ }^{\Delta \rightarrow N e^{+} e^{-}}\left(M, M_{\Delta}\right): 1\right)$ solid line: total width - "Bass", electromagnetic - "Wolf"; 2) dot-dashed line: total width - "Bass", electromagnetic - "Krivoruchenko"; 3) dashed line: total width - "Const", electromagnetic - "Wolf"; 4) dot-dot-dashed line: total width - "Const", electromagnetic - "Krivoruchenko". The variation of $\Gamma_{\Delta}^{t o t}\left(M_{\Delta}\right)$ changes the dilepton yield only marginally as long as the same electromagnetic decay width is used - cases 1),3) and 2),4). The reason can easily be seen from Eq. (16) : the total width $\Gamma_{\Delta}^{t o t}\left(M_{\Delta}\right)$ enters in the numerator of spectral function (Eq. 10) and in the denominator of the branching ratio and thus cancels. The only remaining dependence comes from the denominator of Eq. (10) but far from the pole mass $\Gamma_{\Delta}^{t o t}\left(M_{\Delta}\right)$ this term is small as compared to the other part of the denominator. Oppositely, for a fixed total width $\Gamma_{\Delta}^{t o t}\left(M_{\Delta}\right)$ the variation of $\frac{d \Gamma}{d M}{ }^{\Delta \rightarrow N e^{+} e^{-}}\left(M, M_{\Delta}\right)$ leads to differences of the dilepton yield up to the factor of 3 for high invariant masses - cases 1),2) and 3),4).

Thus we can conclude that different assumptions on the total width $\Gamma_{\Delta}^{t o t}\left(M_{\Delta}\right)$ have little influence on the invariant mass distribution of dileptons whereas the lack of knowledge of $\frac{d \Gamma}{d M}{ }^{\Delta \rightarrow N e^{+} e^{-}}\left(M, M_{\Delta}\right)$ introduces an uncertainty of up to a factor of three for the dilepton yield from $\Delta$ decay at large invariant masses.

Different assumptions have been made of how to relate $\Gamma_{\Delta}^{t o t}\left(M_{\Delta}\right)$ to the lifetime of the $\Delta$. We do not discuss here the rational behind the different approaches. Rather we concentrate on the consequences for the dilepton yield. In 

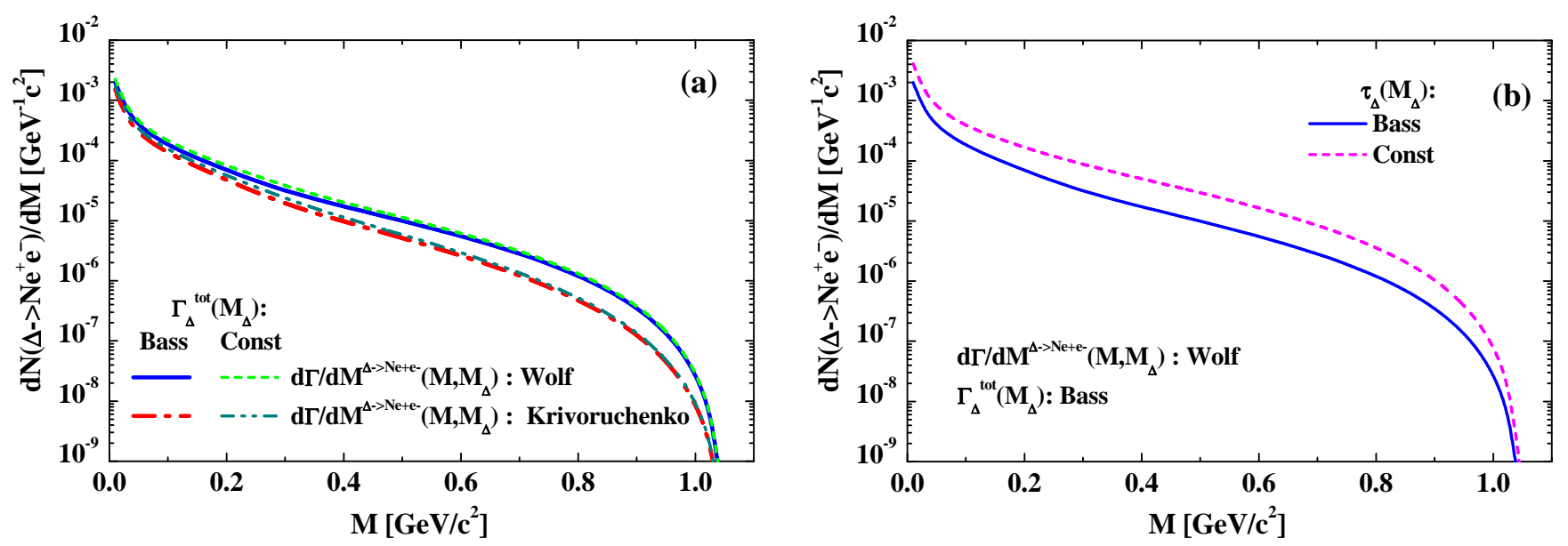

FIG. 40: (Color online) Left(a): the dilepton yield as a function of invariant dilepton mass for the 2 parametrization of the total width $\Gamma_{\Delta}^{t o t}\left(M_{\Delta}\right) 2$ models for the partial electromagnetic decay width $\left.\frac{d \Gamma}{d M}{ }^{\Delta \rightarrow N e^{+} e^{-}}\left(M, M_{\Delta}\right): 1\right)$ solid line: total width - "Bass", electromagnetic - "Wolf"; 2) dot-dashed line: total width - "Bass", electromagnetic - "Krivoruchenko" 3) dashed line: total width - "Const", electromagnetic - "Wolf" 4) dot-dot-dashed line: total width - "Const", electromagnetic - "Krivoruchenko". Right(b): the dilepton yield as a function of invariant dilepton mass for the 2 assumptions of the life time $\tau_{\Delta}\left(M_{\Delta}\right)$ : solid line "Bass", dashed line - "Const" life time, while using the "Bass" total width for spectral function and the "Wolf" model for the partial electromagnetic decay width in both cases.

HSD the total width for the $\Delta$ production (i.e. that which enters the spectral function $A_{\Delta}\left(M_{\Delta}\right)$ ) is the same as the width used to determine the life time Eq. (15). In this case, we have a cancellation of the total width in Eq. (16) as discussed above, which leads to the low sensitivity of dilepton spectra to different $\Gamma_{\Delta}^{t o t}\left(M_{\Delta}\right)$. In the UrQMD model (cf. e.g. the corresponding discussion in Ref. 71], Section 3.3.4), on the contrary, the width used for the $\Delta$ production differs from that in the life time definition (15), so there is no cancellation of the widths any more, rather the ratios of the two widths enters the Eq. (16).

In the right part (b) of Fig. 40 we demonstrate the consequences of the different life time definitions. We employ in all cases the "Wolf" parametrization of $\frac{d \Gamma}{d M}{ }^{\Delta \rightarrow N e^{+} e^{-}}\left(M, M_{\Delta}\right)$ and $\Gamma_{\Delta}^{t o t}\left(M_{\Delta}\right)$ of "Bass" but vary the description of the life time. The full blue line shows the dilepton yield under the assumption that $\Gamma_{\Delta}^{t o t}\left(M_{\Delta}\right)$ of "Bass" determines the lifetime (Eq. (15)) whereas the dashed red line shows the result assuming that for the calculation of the life time (Eq. (15)) a constant width of $120 \mathrm{MeV}$ is employed. For a constant width we observe a strong enhancement which is mainly related to the large contribution of the high mass $\Delta$ 's to the dilepton yield. This is illustrated in Fig. 41 where we show the contribution of $\Delta$ 's from different mass ranges to the dilepton yield. The sum of all 4 bins gives the solid curve of fig. 40. One has to keep in mind, however, that in real $N N$ collisions at low energies the high mass tail of the $\Delta$ distribution is strongly suppressed due to the limitation of the phase space.

\section{2. $p p$ and heavy-ion collisions}

Now we extend our study of systematic errors to $p p$ and heavy-ion calculations. For this purpose we use the HSD model.

The $\Delta$ resonances can be produced dominantly in $N N$ or $\pi N$ collisions. The mass distribution of the produced $\Delta$ 's $-d N / d M_{\Delta}\left(s, M_{\Delta}\right)$ is defined by the spectral function $A_{\Delta}\left(M_{\Delta}\right)$ (Eq.(10) ) integrated over the corresponding phase space which depends on the invariant energy $\sqrt{s}$ of the $N N$ or $\pi N$ collisions and the masses of the final associated particles $M_{X}$ (e.g. $N N \rightarrow \Delta+X$ ). At low energies the phase space leads to the suppression of high mass $\Delta$ 's.

We start with the time integrated $\Delta$ mass distribution $d N / d M_{\Delta}$. It is shown in Fig. 42 for central $\mathrm{C}+\mathrm{C}$ collisions at $2 A \mathrm{GeV}$. We display the mass distribution for 2 choices for the total width: The solid line displays the calculation for the "Monitz" width, the short dashed line that for the "Bass" width. For comparison we also show the spectral function $A_{\Delta}\left(M_{\Delta}\right)$ (scaled to the maximum of $d N / d M_{\Delta}$ ) for the both widths. Due to the limited available energy in low energy heavy-ion collisions only a part of the full spectral function can be explored (the absorption and rescattering effects for $\mathrm{C}+\mathrm{C}$ collisions do not distort the initial production shape of $\Delta$ mass distribution too much). This lowers the uncertainties of the predicted dilepton yields related to the very high mass tail of the distribution. Going to higher energies the phase space opens more and more and the high mass tail of spectral function can be populated. This is 


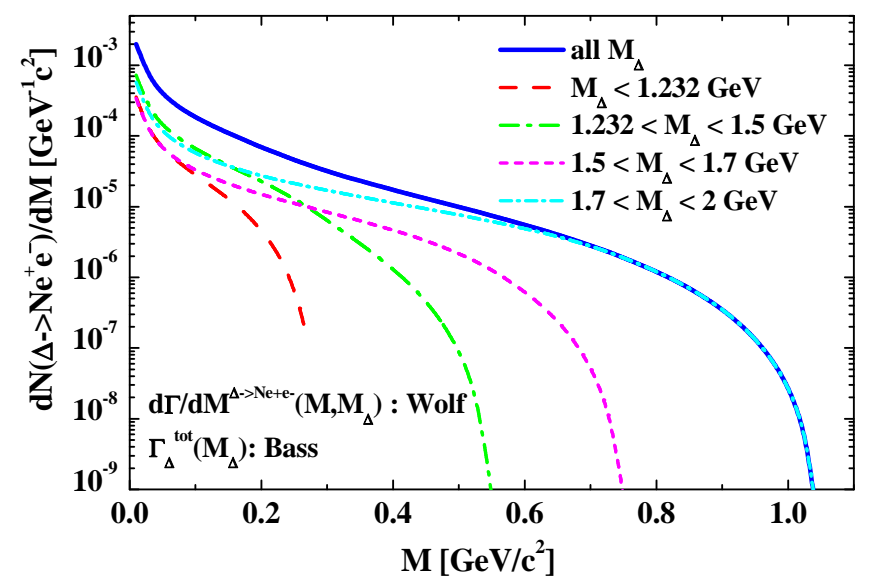

FIG. 41: (Color online) The contribution of $\Delta$ 's from the 4 mass bins to the dilepton yield $d N / d M\left(\Delta \rightarrow N e^{+} e^{-}\right)$: 1 ) $M_{\Delta} \leq 1.232 \mathrm{GeV}$, 2) $1.232 \leq M_{\Delta} \leq 1.5 \mathrm{GeV}$, 3) $1.5 \leq M_{\Delta} \leq 1.7 \mathrm{GeV}$, 4) $1.7 \leq M_{\Delta} \leq 2.0 \mathrm{GeV}$. Calculations are done using the "Bass" total width for spectral function and "Wolf" model for the partial electromagnetic decay width.

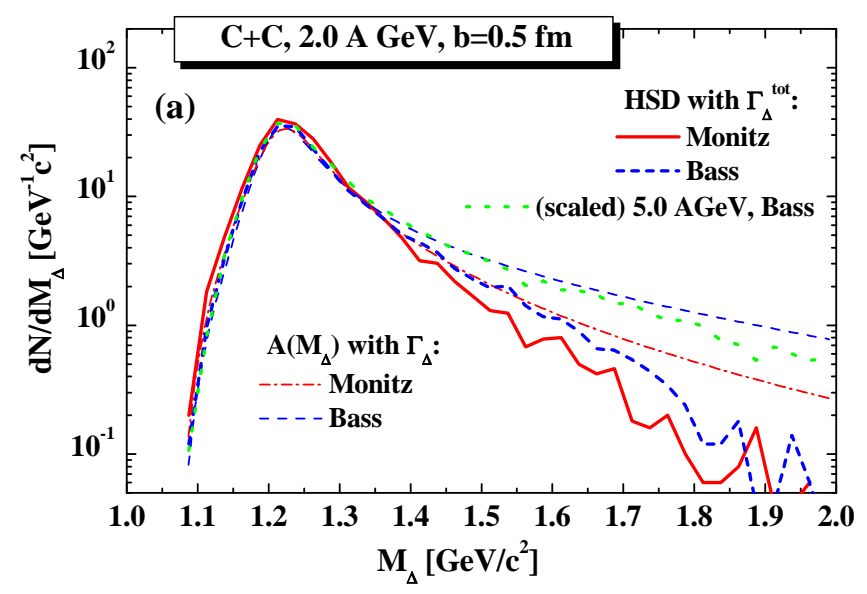

FIG. 42: (Color online) The $\Delta$ mass distribution from HSD for the central C+C collisions at $2 A$ GeV for the 2 model cases for the total width: solid line - "Monitz", short dashed - "Bass" width. The thin dash-dotted and dashed lines show the spectral function $A_{\Delta}\left(M_{\Delta}\right)$ calculated with "Monitz" and "Bass" widths, correspondingly. The dotted line stands for $d N / d M_{\Delta}$ from HSD for C+C collisions at $5.0 A \mathrm{GeV}$ with "Bass" width (scaled to the maximum of $d N / d M_{\Delta}$ at $2 A \mathrm{GeV}$ for easy comparison of the shape of mass distributions)

shown by the dotted line which displays $d N / d M_{\Delta}$ for $\mathrm{C}+\mathrm{C}$ collisions at $5.0 \mathrm{AGeV}$ employing the "Bass" width which we scaled to the maximum of $d N / d M_{\Delta}$ for $\mathrm{C}+\mathrm{C}$ at $2 A \mathrm{GeV}$ for easy comparison of the shape of corresponding mass distributions.

Similar to the left (a) part of Fig. 40 we demonstrate in Fig. 43 the consequences of the variation of the electromagnetic decay width on the differential cross section $d \sigma / d M$ (left (a)) and the transverse momentum spectra (right (b-e)) for 4 different mass bins $(M \leq 0.15 \mathrm{GeV}, 0.15 \leq M \leq 0.47 \mathrm{GeV}, 0.47 \leq M \leq 0.7 \mathrm{GeV}$ and $M \geq 0.7 \mathrm{GeV}$ ) from HSD calculations for $e^{+} e^{-}$production in $p p$ reactions at a bombarding energy of $3.5 \mathrm{GeV}$. The dash-dot-dotted and the dashed lines show the Delta Dalitz contribution and the corresponding total sum of all channels without $p p$ Bremsstrahlung as in Figs. 11/12 - for the "Wolf" electromagnetic decay width. The dash-dotted line stands for the parametrization using "Krivoruchenko" width, the solid line is the corresponding sum. We point out that we have selected the $p p$ reaction at $3.5 \mathrm{GeV}$ here since at this high energy the open phase space is large enough to populate the high mass $\Delta$ 's. Thus, one expects a large deviation in the dilepton mass spectra coming from the high mass tail of the $\Delta$ spectral function - as follows from Figs. 38 and 40(left) - compared to the low energy reactions where the available energy limits the production of heavy $\Delta$ 's. Furthermore, Figure 43 (right) demonstrates the sensitivity of $p_{T}$ distribution to the form of the electromagnetic decay width. In spite that the deviation is bigger for the bin with the largest dilepton masses $(M>0.7 \mathrm{GeV})$, this effect is not visible in the final $p_{T}$ spectra due to the dominant con- 

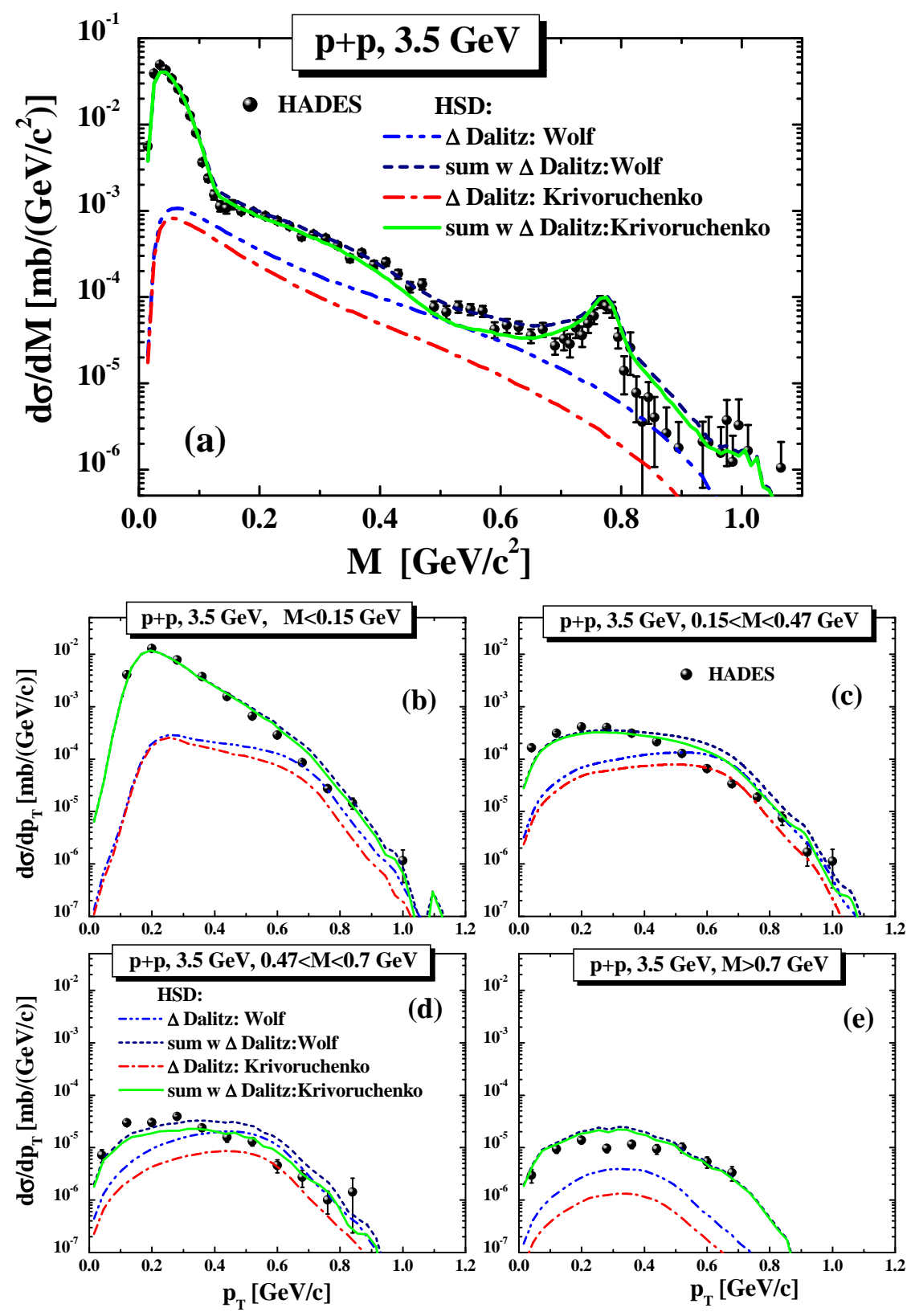

FIG. 43: (Color online) Left (a): The differential cross section $d \sigma / d M$ from HSD calculations for $e^{+} e^{-}$production in $p p$ reactions at a bombarding energy of $3.5 \mathrm{GeV}$ in comparison to the HADES data [57]. Right(b-e): The HSD results for the transverse momentum spectra for $p p$ at $3.5 \mathrm{GeV}$ and for 4 different mass bins: $M \leq 0.15 \mathrm{GeV}, 0.15 \leq M \leq 0.47 \mathrm{GeV}, 0.47$ $\leq M \leq 0.7 \mathrm{GeV}$ and $M \geq 0.7 \mathrm{GeV}$ in comparison to the HADES data [57]. The individual lines similar to the left part. The dash-dot-dotted and the dashed lines shows the Delta Dalitz contribution and the corresponding total sum of all channels without $p p$ Bremsstrahlung as in Figs. 1112 - for the "Wolf" electromagnetic decay width. The dash-dotted line stands for the parametrization using "Krivoruchenko" width, the solid line is the corresponding sum.

tributions from the direct decay of vector mesons. For the lower mass bins $(0.15 \leq M \leq 0.47 \mathrm{GeV}$, and $0.47 \leq M \leq$ $0.7 \mathrm{GeV}$ ) the difference is better observed in the final $p_{T}$ spectra. Thus, the measurement of the $p_{T}$ distributions at various mass bins can help in distinguishing of different models.

We continue with the comparison of the final mass differential dilepton spectra for central $\mathrm{C}+\mathrm{C}$ collision at $2 A \mathrm{GeV}$ - Fig. 44 (left (a)). The legend for the individual lines is the same as in Fig. 43, We observe similar deviations as obtained within the "convolution" model - see the left (a) part of Fig. 40.

Thus, we conclude that the uncertainty of the electromagnetic decay width of the $\Delta$ resonance translates to an uncertainty of about a factor of 1.5 in the dilepton yield from $\Delta$ decays in heavy ion collisions. For large invariant 

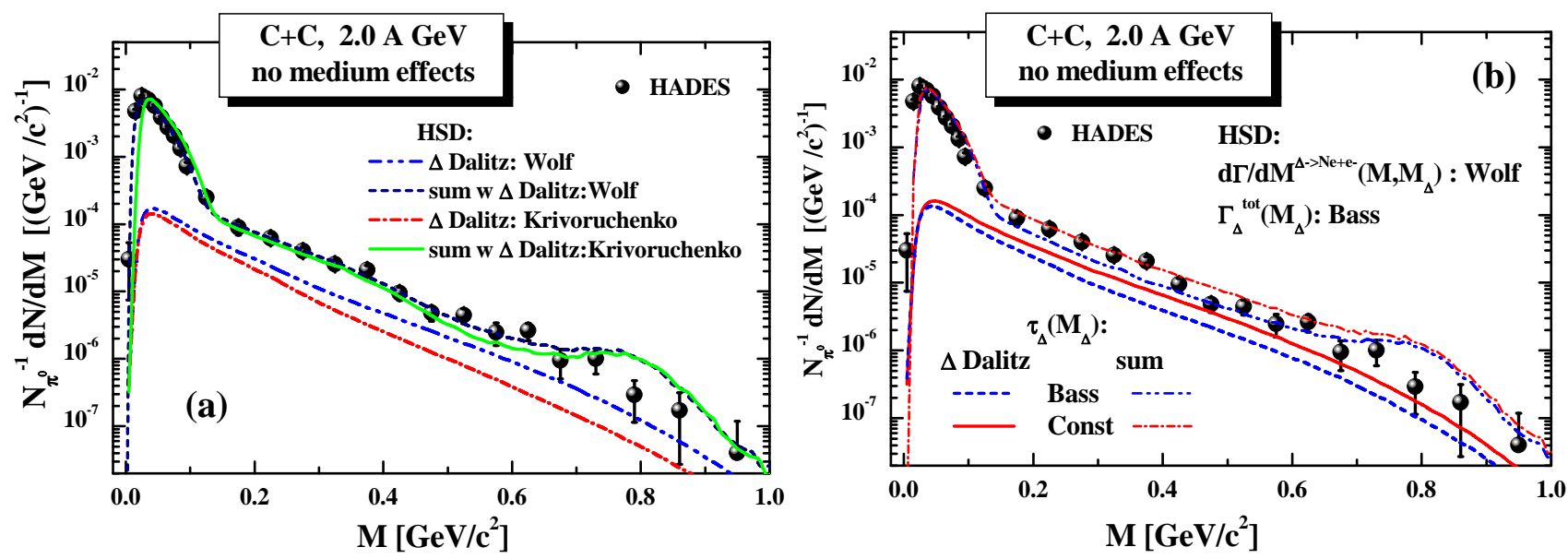

FIG. 44: (Color online) Left (a): The mass differential dilepton spectra - normalized to the $\pi^{0}$ multiplicity - from HSD calculations for central $(\mathrm{b}=0.5 \mathrm{fm}) \mathrm{C}+\mathrm{C}$ collisions at $2 \mathrm{AGeV}$ in comparison to the HADES data 37]. The dash-dot-dotted and the dashed lines shows the Delta Dalitz contribution and the corresponding total sum of all channels as in Fig. 16. for the "Wolf" electromagnetic decay width. The dash-dotted line stands for the parametrization using "Krivoruchenko" width, the solid line is the corresponding sum. Right(b): similar to the left part - the mass differential dilepton spectra as a function of invariant dilepton mass for the 2 model cases of life time $\tau_{\Delta}\left(M_{\Delta}\right)$ : solid line - "Const", dashed line - "Bass" life time, while using the "Bass" total width for the $\Delta$ production and dynamics and "Wolf" model for the partial electromagnetic decay width in both cases.

masses of the $\Delta$ this uncertainty reaches even a factor of 3 . However, these large invariant masses are only populated at beam energies at which $\eta$ production becomes important with the consequence that the $\eta$ Dalitz decay and bremsstrahlung are the dominant sources for dilepton production. Therefore the uncertainty of the dilepton yield from large mass $\Delta$ decay has little influence on the measured total dilepton yield at large invariant masses as well as on the ratio $R(A A / N N)$ - cf. dash-dot-dotted line in Fig. 26 (b).

The simulations for the different assumptions about the life time for the central $\mathrm{C}+\mathrm{C}$ at $2 A \mathrm{GeV}$ are presented in the right part (b) of Fig. 44. The assumptions correspond to that of the right (b) part of Fig. 40. The solid line - "Const" - displays the results assuming a constant life time whereas the dashed line show the result assuming the "Bass" life time. In both cases the "Bass" total width has been employed for the $\Delta$ spectral function and the "Wolf" model has been used for the partial electromagnetic decay width. One can see that the two assumptions about the lifetime yield an uncertainty of a factor of 2 , slightly less than the factor we obtained for elementary reactions. (Fig. 421).

We would like to stress here that the uncertainties in the electromagnetic decay width of $\Delta$ resonance as well as that of total width/life time of the $\Delta$ can be reduced by measuring the dilepton yield in $\pi N$ reactions at different energies. Such a measurement would allow for preciser predictions than presently possible.

\section{Electromagnetic $\Delta-N$ transition formfactor}

The introduction of the electromagnetic $\Delta-N$ transition formfactor $F_{\Delta N}$ for the $\Delta$ Dalitz decay has been studied in Ref. [82] within the GiBUU transport model for $N N$ and $p N b$ reactions. The model from Refs. 94 has been choosen for the $\Delta-N$ transition formfactor which is based on the Vector Dominance Model (VDM) model assuming that the virtual photon is converted first to a $\rho_{0}$ meson, i.e. the transition $\Delta \rightarrow \gamma * N \rightarrow N e^{+} e^{-}$can be considered as $\Delta \rightarrow \gamma^{*} N \rightarrow \rho_{0} N \rightarrow e^{+} e^{-} N$. For that one needs to extrapolate the $\Delta-N$ transition formfactor from the space-like region to the time-like region where its strength is unknown experimentally.

In Fig. 45 we demonstrate the effect of the electromagnetic $\Delta-N$ transition formfactor: the left plot (a) shows the electromagnetic decay width of the $\Delta$ resonance into dileptons $\Delta \rightarrow N e^{+} e^{-}$using the "Krivoruchenko" model for different $\Delta$ masses of $1.232 \mathrm{GeV}$ (a), $1.5 \mathrm{GeV}$ (b) and $1.8 \mathrm{GeV}$ (c): the thick (thin) lines - with (without) the $\Delta-N$ formfactor $F_{\Delta N}$ from Ref. [94]. The right plot (b) displays the dilepton yield using the "convolution" model (Eq. (16)) with the "Bass" total width. One can see that the inclusion of the formfactor leads to an enhancement of the dilepton yield up to a factor of 10 at $M \sim 0.6 \mathrm{GeV}$.

We have investigated the consequences of the electromagnetic $\Delta-N$ transition formfactor for heavy-ion collisions for $\mathrm{C}+\mathrm{C}$ reactions at $2 \mathrm{AGeV}$ where the $\Delta$ channel is one of the dominant channels. Fig. 46 shows the results of 

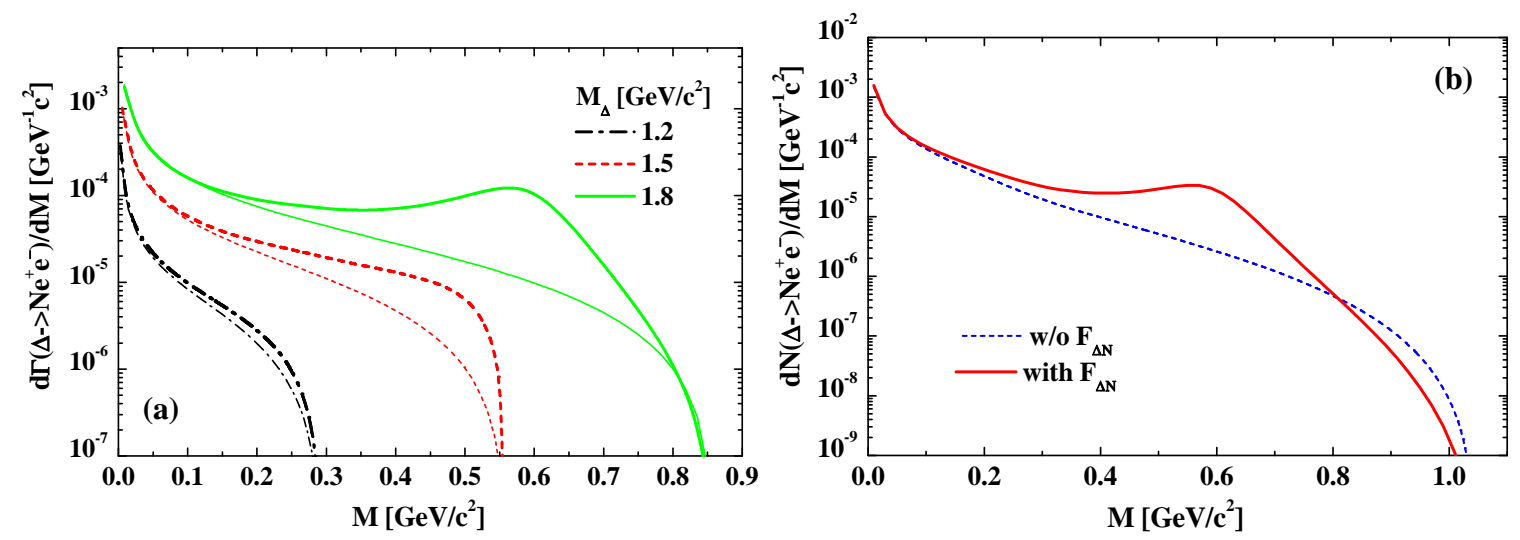

FIG. 45: (Color online) Left (a): the electromagnetic decay width of the $\Delta$ resonance to dileptons $\Delta \rightarrow N e^{+} e^{-}$using the "Krivoruchenko" model for different $\Delta$ masses of $1.232 \mathrm{GeV}$ (a), 1.5 (b) and $1.8 \mathrm{GeV}$ (c): the thick lines - with the $\Delta-N$ formfactor $F_{\Delta N}$ from Ref. 94], the thin lines - without the $\Delta-N$ formfactor. Right (b): the dilepton yield as a function of the invariant dilepton mass using the "Krivoruchenko" model for the electromagnetic decay width and the "Bass" model for the total width: the solid lines - with including the $\Delta-N$ formfactor $F_{\Delta N}$, the dashed line - without the $\Delta-N$ formfactor.

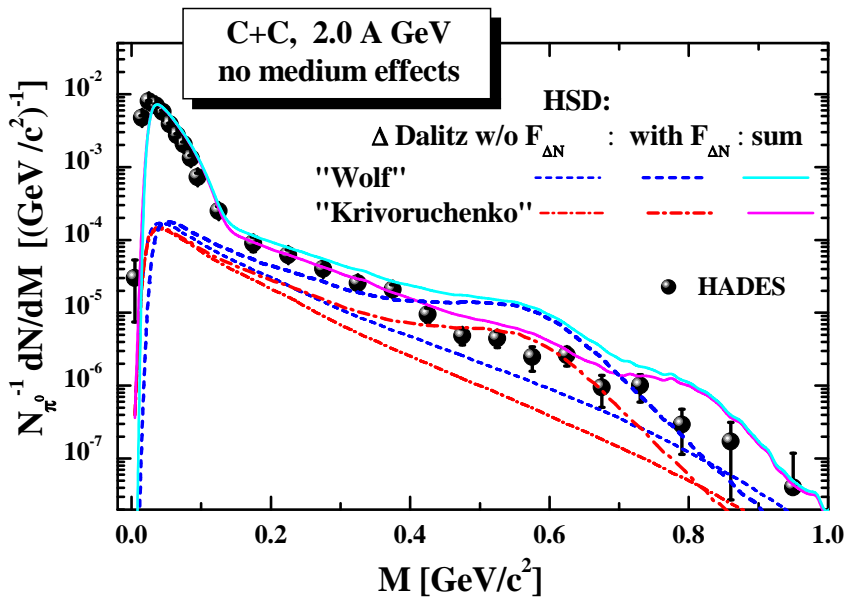

FIG. 46: (Color online) The results of the HSD transport calculation for the mass differential dilepton spectra - normalized to the $\pi^{0}$ multiplicity - for $\mathrm{C}+\mathrm{C}$ at $2.0 \mathrm{AGeV}$ : the thick lines - with the $\Delta-N$ formfactor $F_{\Delta N}$ from Ref. 94], the thin lines without the $\Delta-N$ formfactor. Here the dashed lines corresponds to the "Wolf" model for the electromagnetic decay width, the dash-dotted lines - for the "Krivoruchenko" model.

the HSD calculations for 2 different models (in line with our discussion above on the model uncertainties) for the electromagnetic decay width - "Krivoruchenko" (dashed lines) and "Wolf" (dash-dotted lines) which provide the lower and upper limit for the effects of the form factor for the final spectra (solid lower and upper lines). One can conclude that the introduction of the $\Delta-N$ transition formfactor [94] leads to overestimation of the dilepton yield in heavy-ion collisions, i.e. is not in line with the HADES data.

\section{E. Electromagnetic pion formfactor for the $p n$ bremsstrahlung}

Here we discuss the uncertainties related to the implementation of the electromagnetic pion formfactor $F_{\pi}(M)$ motivated by the Vector Dominance Model (VDM) model for the pn bremsstrahlung contribution as advocated in the OBE model in Ref. [93]. With the help of this formfactor one hopes to account for the dilepton radiation from the internal charged pion exchange line in $p n \rightarrow p n e^{+} e^{-}$processes assuming vector dominance, i.e. that the photon couples to dileptons via a $\rho^{0}$ meson. This diagramm doesn't exist for the $p p$ reaction, so the enhancement should be seen only in $p n$ dilepton yield. There is a debate if the virtual photon converts fully (i.e. by $100 \%$ ) to $\rho$ meson $\left(\gamma^{*} \rightarrow \rho \rightarrow e^{+} e^{-}\right)$[95] or by $50 \%$ only [96] and the rest decays directly into dileptons $\left(\gamma^{*} \rightarrow e^{+} e^{-}\right)$. In Ref. [96] the 

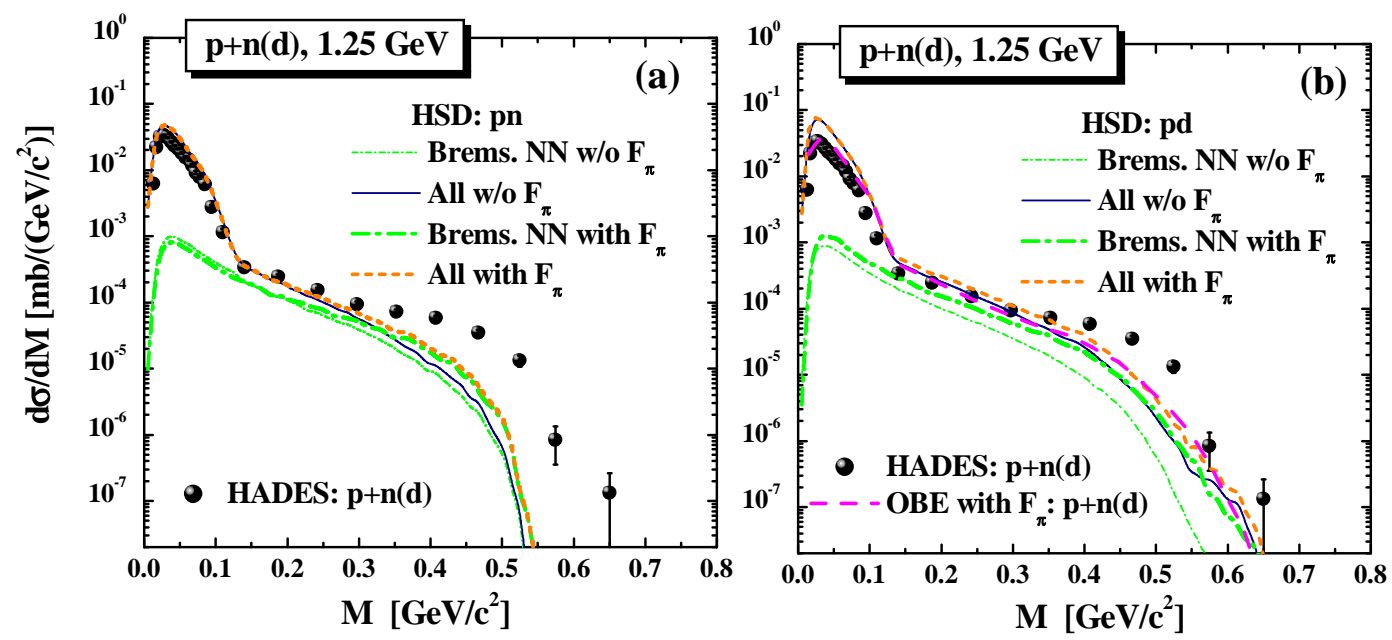

FIG. 47: (Color online) The HSD results for the dilepton differential cross section $d \sigma / d M$ for $p n$ (left plot (a)) and $p d$ (right plot (b)) reactions at $1.25 \mathrm{GeV}$ including the electromagnetic pion formfactor for the bremsstrahlung channel (denoted as "Brems. NN with $F_{\pi}$ ") in comparison to the "standard" HSD calculations without formfactor (denoted as "Brems. NN w/o $F_{\pi}$ ") as in Fig. 6] The dashed line (denoted as "OBE with $F_{\pi}: p+n(d)$ ") shows the OBE results for $p+n(d)$ from Ref. 93].

electromagnetic pion formfactor $F_{\pi}(M)$ has been parametrized as

$$
F_{\pi}\left(M^{2}\right)=\frac{0.4}{1-M^{2} / \lambda^{2}}+\frac{0.6}{1-M^{2} / 2 m_{\rho}^{2}} \frac{m_{\rho}^{2}}{m_{\rho}^{2}-M^{2}-i m_{\rho} \Gamma_{\rho}\left(M^{2}\right)},
$$

where $\lambda^{2}=1.9 \mathrm{GeV}^{2}$. The width $\Gamma_{\rho}\left(M^{2}\right)$ is given in Ref. [96] as

$$
\Gamma_{\rho}^{t o t}(M)=\Gamma_{\rho^{0} \rightarrow \pi \pi} \frac{r_{C}^{2} k^{3}}{M\left(1+r_{C}^{2} k^{2}\right)}
$$

with $k^{2}=M^{2} / 4-m_{\pi}^{2}$. The parameter $r_{C}=2 \mathrm{fm}$ is an interaction radius, $\Gamma_{\rho^{0} \rightarrow \pi \pi}=0.150 \mathrm{GeV}$.

According to the OBE calculations of [93] for the $p n$ reaction at $1.25 \mathrm{GeV}$ the incorporation of the formfactor $F_{\pi}(M)$ leads to the enhancement of the bremsstrahlung contribution and a better agreement with the HADES data for quasi-free $p n$ scattering. Following Ref. [93] we have performed a model study by including the formfactor from [96] in our calculations of $p n$ bremsstrahlung by simply multiplication of the parametrized OBE results from [41] used in HSD by the formfactor of eq. (18). Indeed, this provides an upper limit since we can not distinguish the individual diagramms in our parametrization of the bremsstrahlung cross section. We obtain a good agreement with the full OBE calculations from [93, as shown in Fig. 47, which presents the HSD results for the dilepton differential cross section $d \sigma / d M$ for $p n$ (left plot (a)) and $p d$ (right plot (b)) reactions at $1.25 \mathrm{GeV}$ including the electromagnetic pion formfactor for the bremsstrahlung channel (denoted as "Brems. NN with $F_{\pi}$ ") in comparison to the "standard" HSD calculations without formfactor (denoted as "Brems. NN w/o $F_{\pi}$ ") as in Fig. 6. The dashed line (denoted as "OBE with $\left.F_{\pi}: p+n(d) "\right)$ shows the OBE results for $p+n(d)$ from Ref. 93].

As seen form Fig. 47 the inclusion of the formfactor doesn't explain the experimental HADES quasi-free $p+n(d)$ data. We check now how the formfactor will change the heavy-ion results where we have reliable experimental constraints from the HADES measurements. The HSD results for the dilepton differential cross section $d \sigma / d M$ for $\mathrm{C}+\mathrm{C}$ at $1.0 \mathrm{AGeV}$ (left (a)) $2.0 \mathrm{AGeV}$ (middle (b)) and $\mathrm{Ar}+\mathrm{KCl}$ (right (c)) reactions at $1.75 \mathrm{AGeV}$ are shown in Fig. 48. One can clearly see a sizeable overestimation of the dilepton yields for all systems which brings us to the conclusion that to include a form factor is not supported by the experimental data on heavy-ion collisions. 

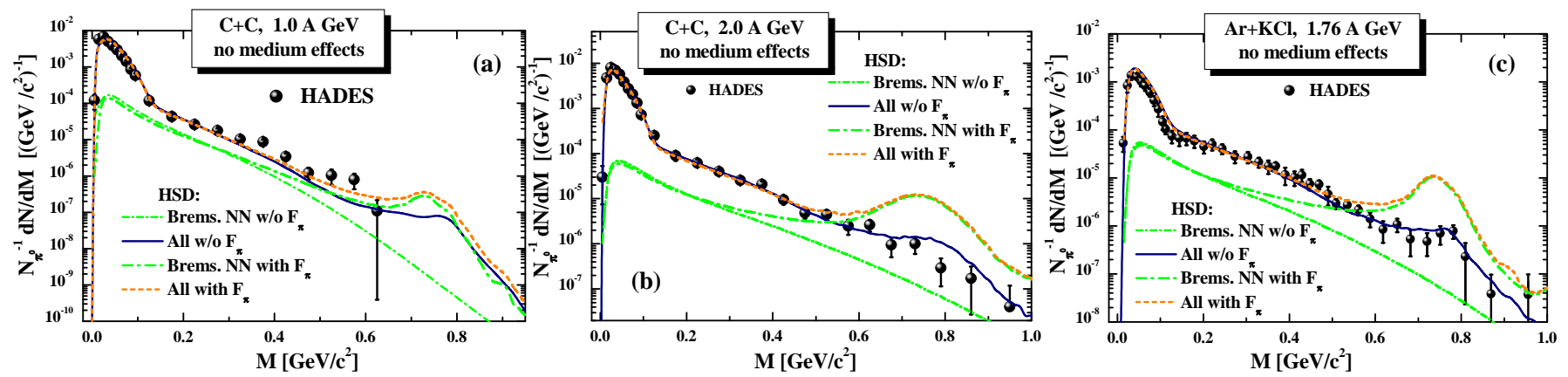

FIG. 48: (Color online) The HSD results for the dilepton differential cross section $d \sigma / d M$ for $\mathrm{C}+\mathrm{C}$ at $1.0 A \mathrm{GeV}$ (left (a)) 2.0 $A \mathrm{GeV}$ (middle (b)) and $\mathrm{Ar}+\mathrm{KCl}$ (right (c)) reactions at $1.75 \mathrm{AGeV}$. The lines description as in Fig. 47.

\section{CONCLUSIONS}

We have studied the production of dileptions in $p p, p n, p A$ and $A A$ collisions at energies between 1 and $3.5 A \mathrm{GeV}$ by comparing the results of three independent transport approaches - HSD, IQMD and UrQMD - with all existing data in this energy domain. These data allowed for the first time to study the cycle of creation and absorption of the $\Delta$ resonance in heavy ion reaction which has been theoretically predicted since long.

Despite of common general ideas of transport approaches which are based on the modeling/parameterizations of baryon-baryon, meson-baryon and meson-meson elementary reactions with further dynamical evolution including the propagation in a self-generated mean-field potential and explicit interactions, the models differ in the actual realization and underlying assumptions where no control from experimental data is available.

Especially for one of the dominant channels for the dilepton production in this energy domain, the $\Delta$ Dalitz decay, the experimental results do not allow for a robust parametrization of the input for these transport theories. Neither the spectral function of the $\Delta$ nor the differential decay width of the $\Delta$ into dileptons are well known. At energies around $1 \mathrm{AGeV}$ the $\Delta$ production is in agreement with the isospin model which assumes that the difference $\Delta$ states are produced according to the isospin Clebsch Gordon coefficients at higher energies, where 2 pion channels contribute substantially, little information is available on population of the different $\Delta$ states. This situation will hopefully change with the planned experiments on dilepton production in $\pi N$ collisions. Such an information would substantially improve the predictive power of transport theories for heavy ion results.

Similar is the situation for the bremsstrahlung contribution which turns out to be the dominant channel for the low energy collisions at $1 \mathrm{AGeV}$. The present OBE models provide different predictions as compared to the softphoton-approximation and do not agree among each other. More precise data, especially on dilepton production in elementary $p p, p n$ and especially $\pi N$ collisions for different energies are need to reduce this systematic error. They would allow for a more reliable predictions of bremsstrahlung in transport approaches.

We stress the importance of providing such constraint form the experimental side since the transport models are the only reliable tool to study the physics of heavy ion collisions at those energies where neither thermal models nor hydrodynamic models are applicable because the created matter is far from equilibrium.

Despite of these uncertainties the results of different transport approaches for the final dilepton yield agree quite well among each other even if there are the deviations in the channel decompositions. The data are in between the systematical error of the transport predictions.

We have started our investigation with the dilepton spectra from elementary reactions which can be described as a superposition of the emission from known dilepton sources. In $p p$ collisions at energies of around $1 \mathrm{GeV}$ dileptons stem dominantly from the $\Delta$ Dalitz decay whereas in $p n$ collisions the bremsstrahlung radiation becomes equally important. At higher energies the $\eta$ production sets in and contributes to the invariant mass range $M<0.55 \mathrm{GeV}$. At higher invariant masses the vector meson decays dominate but the data are presently not precise enough to allow for firm conclusions. New experimental differential data would be very useful to check the underlying model assumptions.

Our study demonstrates that in heavy-ion reactions the dilepton production for invariant masses below $M<0.6$ $\mathrm{GeV}$ cannot be interpreted as a simple convolution of the average dilepton yield from $p n$ and $p p$ collisions times the number of elementary collisions. The presence of a nuclear medium manifests itself in several ways: First of all the Fermi motion of nucleons in nuclei smears out the energy distribution of primary $N N$ collisions substantially. This has a big influence on the particle production at (sub-)threshold energies. The Fermi motion enhances the pion as well as the dilepton yields in $A A$ collisions at threshold energies by up to a factor of two. The enhancement is, 
however, identical for pions and dileptons. Therefore, if the Fermi motion would be the only difference between $A A$ and $N N$ collisions, one could expect that if one normalizes the dilepton yield to the pion multiplicity, as done in the experimental analysis, no enhancement would be observed.

The real situation is quite different: an enhancement of the dilepton yield in $A A$ relative to $N N$ is observed experimentally even if one normalizes the dilepton yield by the $\pi^{0}$ multiplicity. The experimental enhancement is, however, plagued partly by the use of $p d$ collisions instead of $p n$ collisions because in the interesting kinematical regime the $p d$ collisions are true three-body collisions and cannot be interpreted as quasi-free $p n$ reactions. So the 'true' enhancement - as compared to elementary collisions - cannot be inferred from present data for invariant masses above $0.5 \mathrm{GeV}$.

We have analysed this enhancement in detail and found two origins: The first reason is the bremsstrahlung radiation from $p n$ and $p p$ reactions which does not scale with the pion number (i.e. the number of participants) rather with the number of elementary elastic collisions. The second reason is the shining of dileptons from the 'intermediate' $\Delta$ 's, which take part in the $\Delta \rightarrow \pi N$ and $\pi N \rightarrow \Delta$ reaction cycle. This cycle produces a number of generations of $\Delta$ 's during the reaction which increases with the size of the system. At the end only one pion is produced but each intermediate $\Delta$ has contributed to the dilepton yield because emitted dileptons do not get absorbed. This leads to an enhancement of the dilepton yield as compared to the final number of pions. Thus, the enhancement confirms the predictions of transport theories that in heavy-ion collisions several generations of $\Delta$ 's are formed which decay and are recreated by $\pi N \rightarrow \Delta$ reactions. Accordingly, the dilepton data from $A A$ reactions shed light on the $\Delta$ dynamics in the medium.

In the investigated invariant mass range, $M<0.5 \mathrm{GeV}$, we do not find evidence that the observed enhancement of the dilepton yield in heavy-ion collisions over the elementary reactions requires the assumption of 'conventional' in-medium effects like a modification of the spectral functions of the involved hadrons. Theory predicts such a modification for vector mesons and therefore for invariant masses $M>0.5 \mathrm{GeV}$. More precise data are needed to draw robust conclusions on the in-medium modifications in this invariant mass range.

We summarize with the final remark that the ratio of dilepton yields $A A / N N$ is a sensitive observable which allows to penetrate the intermediate phase of the heavy-ion reaction and sheds light on the $\Delta$ dynamics which is not accessible by the hadronic observables. Thus, the HADES data provide the first experimental constraint on this issue in heavy-ion collisions at SIS energies. Moreover, by measuring this ratio at a low bombarding energy one can get access to the bremsstrahlung radiation since it becomes the dominant process there. One expects to observe in this case the scaling of the ratio with the number of binary collisions rather than with the number of pions. A precise measurement of the ratio of dilepton yields in heavy-nuclei collisions (as $\mathrm{Au}+\mathrm{Au}$ or $\mathrm{Pb}+\mathrm{Pb}$ ) and of that in the light systems (as $\mathrm{C}+\mathrm{C}$ ) for invariant masses $M>0.5 \mathrm{GeV}$ will help to obtain information on the in-medium modification of the spectral function of vector mesons.

\section{Acknowledgements}

The authors are grateful for fruitful discussions with W. Cassing, C. Hartnack, S. Endres, L. Fabbietti, M. Gumberidze, F. Krizek, Y. Leifels, O. Linnyk, A. Rustamov, J. Stroth, P. Salabura, H. van Hees, M. Weber and J. Weil. Our special thanks go to T. Galatyuk and R. Holzmann for the continuous help and many useful advices concerning the experimental data and filtering procedure. E.B. and S.V. acknowledge financial support through the "HIC for FAIR" framework of the "LOEWE" program.

[1] Z. Fodor and C. Hoelbling, Rev. Mod. Phys. 84 (2012)449.

[2] G. E. Brown and M. Rho, Phys. Rev. Lett. 66 (1991) 2720.

[3] G. E. Brown, C. H. Lee, M. Rho and V. Thorsson, Nucl. Phys. A 567 (1994) 937.

[4] T. Hatsuda, S. H. Lee and H. Shiomi, Phys. Rev. C 52 (1995) 3364.

[5] M. Asakawa, C. M. Ko, P. Lévai, X. J. Qiu, Phys. Rev. C46 (1992) R1159.

[6] M. Herrmann, B. Friman, W. Nörenberg, Nucl. Phys. A560 (1993) 411.

[7] G. Chanfray, P. Schuck, Nucl. Phys. A545 (1992) 271c.

[8] F. Klingl, W. Weise, Nucl. Phys. A 606 (1996) 329; F. Klingl, N. Kaiser, W. Weise, Nucl. Phys. A624 (1997) 527

[9] R. Rapp, G. Chanfray and J. Wambach, Phys. Rev. Lett. 76 (1996) 368.

[10] R. Rapp, G. Chanfray and J. Wambach, Nucl. Phys. A 617 (1997) 472.

[11] W. Peters, M. Post, H. Lenske, S. Leupold, and U. Mosel, Nucl. Phys. A 632 (1998) 109.

[12] S. Leupold, W. Peters, U. Mosel, Nucl. Phys. A628 (1998) 311.

[13] L. Tolós, A. Ramos, and A. Polls, Phys. Rev. C 65 (2002) 054907. 
[14] L. Tolos, R. Molina, E. Oset and A. Ramos, Phys. Rev. C 82 (2010), 045210 (2010).

[15] G. Agakichiev et al. [CERES Collaboration], Eur. Phys. J. C 41 (2005) 475.

[16] J. Seixas et al. [NA60 Collaboration], J. Phys. G 34 (2007) S1023; S. Damjanovic et al., Nucl. Phys. A 783, 327c (2007); R. Arnaldi et al., Eur. Phys. J C61, 711 (2009).

[17] H. van Hees and R. Rapp, J. Phys. G 34 (2007) S1051.

[18] C. Gale and S. Turbide, Nucl. Phys. A 783 (2007) 35.

[19] T. Renk and J. Ruppert, arXiv:hep-ph/0605130.

[20] E.L. Bratkovskaya, O. Linnyk, and W. Cassing, Phys. Lett. B670 (2009) 428.

[21] O. Linnyk, W. Cassing, J. Manninen, E. L. Bratkovskaya and C. M. Ko, Phys. Rev. C 85 (2012) 024910.

[22] E. Santini, J. Steinheimer, M. Bleicher, and S. Schramm, Phys. Rev. C 84 (2011) 014901.

[23] H. S. Matis et al. [DLS Collaboration], Nucl. Phys. A 583 (1995) 617C.

[24] W. K. Wilson et al. [DLS Collaboration], Phys. Rev. C 57 (1998) 1865.

[25] W. K. Wilson et al. [DLS Collaboration], Phys. Lett. B 316 (1993) 245.

[26] R. J. Porter et al. [DLS Collaboration], Phys. Rev. Lett. 79 (1997) 1229.

[27] G. Wolf, W. Cassing and U. Mosel, Prog. Part. Nucl. Phys. 30 (1993) 273; Nucl. Phys. A 552 (1993) 549; Nucl. Phys. A 545 (1992) 139C.

[28] E. L. Bratkovskaya, W. Cassing and U. Mosel, Phys. Lett. B 376 (1996) 12.

[29] L. Xiong, Z. G. Wu, C. M. Ko and J. Q. Wu, Nucl. Phys. A 512 (1990) 772.

[30] C. Ernst, S. A. Bass, M. Belkacem, H. Stoecker and W. Greiner, Phys. Rev. C 58 (1998) 447.

[31] E. L. Bratkovskaya, W. Cassing, R. Rapp, and J. Wambach, Nucl. Phys. A634 (1998) 168.

[32] C. Fuchs, A. Faessler, D. Cozma, B. V. Martemyanov and M. I. Krivoruchenko, Nucl. Phys. A 755 (2005) 499.

[33] E. L. Bratkovskaya and C. M. Ko, Phys. Lett. B445 (1999) 265.

[34] G. Agakishiev et al. [HADES Collaboration], Phys. Lett. B 663 (2008) 43.

[35] Y. C. Pachmayer et al. [HADES Collaboration], J. Phys. G G 35 (2008) 104159.

[36] M. Sudol et al. [HADES Collaboration], Eur. Phys. J. C 62 (2009) 81.

[37] G. Agakishiev et al. [HADES Collaboration], Phys. Lett. B 690 (2010) 118.

[38] K. Lapidus et al. [HADES Collaboration], arXiv:0904.1128 [nucl-ex].

[39] G. Agakishiev et al. [HADES Collaboration], Phys. Rev. C 84 (2011) 014902.

[40] E. L. Bratkovskaya and W. Cassing, Nucl. Phys. A 807 (2008) 214.

[41] L. P. Kaptari and B. Kämpfer, Nucl. Phys. A 764 (2006) 338.

[42] M. Schäfer, T. S. Biro, W. Cassing and U. Mosel, Phys. Lett. B 221 (1989) 1.

[43] R. Shyam and U. Mosel, Phys. Rev. C 67 (2003) 065202.

[44] F. de Jong and U. Mosel, Phys. Lett. B 392 (1997) 273.

[45] M. Thomere, C. Hartnack, G. Wolf and J. Aichelin, Phys. Rev. C 75 (2007) 064902.

[46] H. W. Barz, B. Kämpfer, G. .Wolf and M. Zetenyi, arXiv:0910.1541 [nucl-th].

[47] E. L. Bratkovskaya and W. Cassing, Nucl. Phys. A 619 (1997) 413.

[48] W. Cassing and E. L. Bratkovskaya, Phys. Rept. 308 (1999) 65.

[49] W. Ehehalt and W. Cassing, Nucl. Phys. A 602 (1996) 449.

[50] K. Weber, B. Blaettel, W. Cassing, H. C. Doenges, V. Koch, A. Lang and U. Mosel, Nucl. Phys. A 539 (1992) 713.

[51] B. Andersson, G. Gustafson and H. Pi, Z. Phys. C 57 (1993) 485.

[52] I. Fröhlich et al., PoS ACAT2007 (2007) 076, arXiv:0708.2382 [nucl-ex].

[53] Gy. Wolf et al., Nucl. Phys. A 517 (1990) 615.

[54] E. L. Bratkovskaya, W. Cassing and U. Mosel, Nucl. Phys. A 686 (2001) 568.

[55] W. Cassing and S. Juchem, Nucl. Phys. A 665 (2000) 377; ibid. A 672(2000) 417.

[56] W. Cassing, V. Metag, U. Mosel, and K. Niita, Phys. Reports 188 (1990) 363.

[57] G. Agakishiev et al., [HADES Collaboration], Eur. Phys. J A48 (2012) 64.

[58] H. Calén et al., Phys. Rev. C 58 (1998) 2667.

[59] P. Moskal et al., Prog. Part. Nucl. Phys. 49 (2002) 1.

[60] P. Moskal et al., Int. J. Mod. Phys. A 20(2005) 1880.

[61] G. Agakishiev et al., [HADES Collaboration], Eur. Phys. J A48 (2012), 74.

[62] G. Agakishiev et al., [HADES Collaboration], Phys. Rev. C85 (2012), 054005.

[63] Landolt-Börnstein, New Series, ed. H. Schopper, I/12 (1988).

[64] F. Balestra et al., Phys. Rev. C 63 (2001) 024004; Phys. Rev. Lett. 89 (2002) 092001.

[65] S. Teis, W. Cassing, M. Effenberger, A. Hombach, U. Mosel, and Gy. Wolf, Z. Phys. A 356 (1997) 421; Z. Phys. A 359 (1997) 297.

[66] P. Adlarson et al. [WASA-at-COSY Collaboration], Phys. Lett. B 706 (2012) 256.

[67] L. Alvarez-Ruso, E. Oset and E. Hernandez, Nucl. Phys. A 633 (1998) 519.

[68] E. Oset, J. A. Gomez Tejedor, F. Cano, J. C. Nacher, S. Kamalov, L. Alvarez-Ruso and E. Hernandez, Few Body Syst. Suppl. 11 (1999) 275.

[69] X. Cao, B. -S. Zou and H. -S. Xu, Phys. Rev. C 81 (2010) 065201.

[70] O. Buss, T. Gaitanos, K. Gallmeister, H. van Hees, M. Kaskulov, O. Lalakulich, A. B. Larionov and T. Leitner et al., Phys. Rept. 512 (2012) 1.

[71] S.A. Bass et al., Prog. Part. Nucl. Phys. 42 (1998) 279.

[72] M. Bleicher et al., J. Phys. G 25 (1999) 1859. 
[73] C. Hartnack, R. K. Puri, J. Aichelin, J. Konopka, S. A. Bass, H. Stoecker and W. Greiner, Eur. Phys. J. A 1 (1998) 151.

[74] C. Hartnack, H. Oeschler, Y. Leifels, E. L. Bratkovskaya and J. Aichelin, Phys. Rept. 510 (2012) 119.

[75] J. W. Harris, G. Odyniec, H. G. Pugh, L. S. Schroeder, M. L. Tincknell, W. Rauch, R. Stock and R. Bock et al., Phys. Rev. Lett. 58 (1987) 463.

[76] W. Reisdorf and Y. Leifels, private communication.

[77] W. Reisdorf et al. [FOPI Collaboration], Nucl. Phys. A 781 (2007) 459.

[78] M. Antinucci et al., Lett. al Nuovo Cimento, 6 (1973) 121; M. Gaździcki and D. Röhrich, Z. Phys. C 71 (1996) 55; J. Bächler et al. (NA49 Collab.), Nucl. Phys. A 661 (1999) 45.

[79] M. Weber et al., [HADES Collaboration], J. Phys.: Conf. Ser. 316 (2011) 012007; G. Agakishiev et al., Phys. Lett. B 715 (2012) 304.

[80] M. Lacombe et al., Phys. Lett. B 101 (1981) 139.

[81] P. Danielewicz and G.F. Bertsch, Nucl. Phys. A 533 (1991) 712.

[82] J. Weil, H. van Hees, and U. Mosel, Eur. J. Phys. A 48 (2012) 111.

[83] D. Schumacher, S. Vogel and M. Bleicher, Acta Phys. Hung. A 27 (2006) 451.

[84] K. Schmidt, E. Santini, S. Vogel, C. Sturm, M. Bleicher, and H. Stöcker, Phys. Rev. C 79 (2009) 064908.

[85] S. Endres and M. Bleicher, to be published in the Proceedings of the FAIRNESS-2012 Workshop; S. Endres, M. Bleicher et al., work in progress.

[86] G. Agakishiev et al. [HADES Collaboration], Eur. Phys. J. A 40 (2009) 45.

[87] S. A. Bass, M. Hofmann, C. Hartnack, H. Stoecker and W. Greiner, Phys. Lett. B 335 (1994) 289.

[88] The HADES Collaboration web-cite: http://www-hades.gsi.de/

[89] H. F. Jones and M.D. Scadron, Ann. Phys. 81 (1973) 1.

[90] M. I. Krivoruchenko and A. Faessler, Phys. Rev. D65 (2002) 017502.

[91] M. Zatenyi and Gy. Wolf, Heavy Ion Phys. 17 (2003) 27.

[92] J.H. Koch, E.J. Monitz and N. Ohtsuka, Ann. Phys. 154 (1984) 99.

[93] R. Shyam and U. Mosel, Phys. Rev. C 82 (2010) 062201.

[94] F. Iachello, A.D. Jackson, and A. Lande, Phys. Lett. B 43 (1973) 191; F. Iachello and Q. Wang, Phys. Rev. C69 (2004) 055204 .

[95] T. Ericson and W. Weise, Pions and Nuclei, Clarendon, Oxford, 1988.

[96] G. E. Brown, M. Rho, and W. Weise, Nucl. Phys. A454, 669 (1986). 